\title{
SOME GENERALIZATIONS OF QUASI-PROJECTIVE MODULES
}

\section{QUASI-PROJEKTIF MODÜLLERIN BAZI GENELLEMELERI}

BERKE KALEBOĞAZ

PROF. DR. DERYA KESKİN TÜTÜNCÜ

Supervisor

Submitted to the Institute of Sciences of Hacettepe University as a Partial Fulfillment to the Requirements

for the Award of the Degree of Doctor of Philosophy in Mathematics 
This work named "Some Generalizations of Quasi-projective Modules" by BERKE KALEBOĞAZ has been approved as a thesis for the Degree of DOCTOR OF PHILOSOPHY IN MATHEMATICS by the below mentioned Examining Committee Members.

Prof. Dr. A. Çiğdem ÖZCAN

Head

Prof. Dr. Derya KESKIN TÜTÜNCÜ

Supervisor

Assoc. Prof. Dr. Evrim AKALAN

Member

Assoc. Prof. Dr. Nil ORHAN ERTAŞ

Member

Assoc. Prof. Dr. Noyan Fevzi ER

Member

This thesis has been approved as a thesis for the Degree of DOCTOR OF PHILOSOPHY IN MATHEMATICS by Board of Directors of the Institute for Graduate Studies in Science and Engineering.

Prof. Dr. Fatma SEVINN DÜZ

Director of the Institute of

Graduate Studies in Science 
"This dissertation is dedicated to my husband Hüseyin Kaleboğaz with great love." 


\section{ETHICS}

In this thesis study, prepared in accordance with the spelling rules of Institute of Graduate Studies in Science of Hacettepe University.

I declare that

- all the information and documents have been obtained in the base of the academic rules

- all audio-visual and written information and results have been presented according to the rules of scientific ethics

- in case of using other Works, related studies have been cited in accordance with the scientific standards

- all cited studies have been fully referenced

- I did not do any distortion in the data set

- and any part of this thesis has not been presented as another thesis study at this or any other university.

$26 / 12 / 2014$

BERKE KALEBOĞGZ 


\author{
ABSTRACT \\ SOME GENERALIZATIONS OF QUASI-PROJECTIVE MODULES \\ Berke KALEBOĞAZ \\ Doctor of Philosophy, Department of Mathematics \\ Supervisor: Prof. Dr. Derya KESKİN TÜTÜNCÜ \\ December 2014, 88 pages
}

Our main goal in this dissertation is to investigate semi-projective modules, direct projective modules and SGQ-projective modules, which are generalizations of quasi-projective modules and to discover new properties and new characterizations of these modules.

This dissertation consists of five chapters. In the first chapter, we give preliminary notions and some results that are used in the further sections.

The second chapter is devoted to semi-projective modules. First, we study some basic properties of such modules and provide some new characterizations. Next, direct sum and direct summand properties of semi-projective modules are investigated. In particular, we are interested in direct sums of semi-projective modules over Ore domains. In this chapter, we also define semi-projective cover of a module and give some new characterizations of semiperfect, perfect, semihereditary, hereditary and semisimple rings by using semi-projective modules and semi-projective covers. Finally we investigate rings over which every submodule of semi-projective right $R$-module is semi-projective.

In the third chapter, we define a new concept, namely, SGQ-projective modules, which is another generalization of quasi-projective modules. Some properties of SGQ-projective modules and its endomorphism rings are studied. We characterize semisimple rings by means of SGQ-projective modules.

In the fourth chapter, we first investigate some connections between the notions of epi projectivity, epi $\mathcal{K}$-projectivity, epi $\mathcal{K}^{*}$-projectivity. Then the characterizations of semisimple rings in terms of quasi-epi $\mathcal{K}$-projective, quasi-epi $\mathcal{K}^{*}$-projective 
modules are given. Finally, we investigate the relationship between quasi(-epi) $\mathcal{K}^{*}$ projective modules and Hopfian modules.

The last chapter, Chapter 5, is concerned with modules over formal triangular matrix rings. First, we give detailed background about modules, submodules, quotient modules over triangular matrix rings from related papers. In this last chapter, our results focus on relative projectivity and lifting properties of modules.

Keywords: Ore Domain, semi-projective module, semi-projective cover, SGQ-projective module, epi $\mathcal{K}$-projective module, formal triangular matrix ring. 


\title{
QUASI-PROJEKTİF MODÜLLERİN BAZI GENELLEMELERİ
}

\author{
Berke KALEBOĞAZ \\ Doktora, Matematik Bölümü \\ Tez Danışmanı: Prof. Dr. Derya KESKİN TÜTÜNCÜ \\ Aralık 2014, 88 sayfa
}

$M$ bir sağ $R$-modül olsun. Eğger $M$ modülü $M$-projektif ise, $M$ ' ye quasi-projektif modül denir. Quasi-projektif modüller [11], [12], [14], [16], [17], [18], [35], [57] nolu makalelerde detaylı bir şekilde incelenmiştir. Bu tezin amacı quasi-projektif modüllerin genellemeleri olan semi-projektif, direk projektif ve SGQ-projektif modülleri incelemek ve bu modüllerin bazı yeni özelliklerini ve karakterizasyonlarını vermektir.

Bu tez beş ana başlıktan oluşmaktadır. Tezin birinci bölümünde, çalışma boyunca ihtiyaç duyulacak bazı temel kavramlar ve sonuçlar verilmiştir. Bu çalışmada $R$ aksi belirtilmedikçe birimli, değişmeli olması gerekmeyen ve birleşmeli bir halkayı temsil etmektedir. Ayrıca modüller aksi belirtilmedikçe birimsel sağ $R$-modüllerdir.

Tezin ikinci bölümü tamamen semi-projektif modüllere ayrılmıştır ve bu bölüm beş alt bölümden oluşmaktadır. Birinci alt bölümde semi-projektif modüllerin bazı temel özellikleri ve karakterizasyonları verilmiştir.

Literatüre bakıldığı zaman semi-projektif modüller bugünkü kullanılan şekli ile ilk olarak 1991 yılında R. Wisbauer tarafından tanımlanmıştır. R. Wisbauer [54, p. 260] nolu çalışmada endomorfizmalar halkası $S$ ile gösterilen bir modülün semiprojektif olabilmesi için gerekli ve yeterli koşulun her $\alpha: M \longrightarrow M$ için $\alpha S=$ $\operatorname{Hom}_{R}(M, \alpha(M))$ olduğunu ispatlamıştır. Ayrıca, eğer $M$ devirli altmodülleri için azalan zincir kuralını sağlayan bir sonlu üreteçli, semi-projektif $R$-modül ise $M$ ' nin endomorfizmalar halkasının da devirli sol idealleri için azalan zincir kuralını sağladı̆̆ını ve eğer $M$ artin, semi-projektif bir modül ise $M^{\prime}$ nin endomorfizmalar halkasının semi-primary olduğunu göstermiştir. 2002 yılında, H. Tansee ve S. Wongwai $M$-principally injektif modüllerin duali olarak $M$-principally projektif modülleri 
tanımlamışlardır ve [52, Theorem 2.7] nolu çalışmalarında bir $M$ modülünün $M$ principally projektif olabilmesi için gerekli ve yeterli koşulun onun semi-projektif olması olduğunu ispatlamışlardır. 2007' de, A. Haghany ve M. R. Vedadi [25] semiprojektif retractable modülleri ve onların endomorfizmalar halkalarını incelemişlerdir. 'Bir modüle göre' semi-projektiflik kavramı, 2008 yılında [53] nolu çalışmada X. Wang and J. Chen tarafından ortaya atılmıştır. Ĕğer $N^{\prime}$ den $N^{\prime} \operatorname{nin} M$-devirli altmodülüne olan bir homomorfizma $N^{\prime}$ den $M^{\prime}$ ye bir homomorfizmaya yükseliyorsa, $N$ modülüne $M$-semi-projektif demişlerdir. Bu tanımda $N=M$ alınırsa $M$ semiprojektif olur. Bu tanım [52] numaralı makalede H. Tansee ve S. Wongwai' nin verdiği $M$-principally projektif modül tanımına denktir. Ayrıca onlar bu çalışmada eğer $N$ bir $M$-semi-projektif modül ise $M^{\prime}$ nin herhangi bir $K$ altmodülü için $N^{\prime} \operatorname{nin}$ bir $M / K$-semi-projektif modül olduğunu ve $N^{\prime}$ nin herhangi bir $P$ diktoplananı için $P^{\prime}$ nin $M$-semi-projektif modül olduğunu ispatlamışlardır. 2011 yılında, V. Kumar, A. J. Gupta, B. M. Pandeya and M. K. Patel semi-projektif modüllerin bir genellemesi olarak $M$-SP-projektif modülleri çalışmışlardır.

Tezin bu ilk alt bölümünde her tekil olmayan CS(extending) modülün ve her Rickart modülün bir semi-projektif modül olduğu ispatlanmıştır. Bilindiği gibi bir $M$ sağ- $R$-modülü için aşağıdaki hiyerarşi her zaman vardır;

$\mathrm{M}$ quasi-projektif $\Rightarrow \mathrm{M}$ semi-projektif $\Rightarrow \mathrm{M}$ direk projektif.

Eğer $R$ bir Dedekind halka ve $M$ modülü devirli altmodüllerinin dik toplamı şeklinde ise yukarıdaki gerektirmelerin çift yönlü olduğu bu tezde ispatlanmıştır. Yani her sonlu üreteçli direk projektif $\mathbb{Z}$-modül quasi-projektiftir.

Ayrıca eğer $R$ halkası bir asal PI-halka ve $M$ bir bölünebilir $R$-modül ise, o zaman aşağıdaki çift yönlü gerektirmelerin varlı̆̆ı verilmiştir;

$\mathrm{M}$ semi-projektif $\Leftrightarrow \mathrm{M}$ direk projektif $\Leftrightarrow \mathrm{M}$ semi-Hopfian $\Leftrightarrow \mathrm{M}$ tekil olmayan.

Bu ilk alt bölümde semi-projektif bir modülün her dik toplananının semi-projektif olduğu fakat dik toplamlarının semi-projektif olmadığ (hatta direk projektif bile olmadığı) gösterilmiştir. I indeks kümesindeki her $i \neq j$ için $\operatorname{Hom}_{R}\left(M_{i}, M_{j}\right)=0$ koşulu sağlanıyorsa $M=\bigoplus_{i \in I} M_{i}$ semi-projektiftir ancak ve ancak her bir $i \in I$ için $M_{i}$ semi-projektiftir sonucu elde edilmiştir. 
Bu bölümün ikinci alt bölümünde semi-projektif modüllerin dik toplamları Ore bölgeleri üzerinde çalışılmıştır. Ĕ̆er $R$, kesirler cismi $Q$ olan bir să̆ Ore bölgesi ise $Q_{R}$ ' nin her altmodülünün semi-projektif olduğu ispatlanmıştır. Buna ek olarak, eğer $R$, kesirler cismi $Q \neq R$ olan bir sağ Ore bölgesi ve $X, g\left(X_{R}\right)<g\left(Q_{R}\right)$ koşulunu sağlayan projektif bir sağ $R$-modül ise $X \oplus Q$ semi-projektiftir. Burada $g\left(M_{R}\right)$, kardinalitesi $\kappa$ olan bir $\Lambda$ indeks kümesi ve $M=\Sigma_{(\lambda \in \Lambda)} m_{\lambda} R$ olacak şekildeki $m_{\lambda} \in$ $M$ elemanları var olan en küçük kardinal olan $\kappa$ ' dır. Bu ispatlanan teoremin çok kullanışlı üç tane sonucu vardır. Bunlardan ilki; eğer $R$ kesirler cismi $Q \neq R$ olan bir sağ Ore bölgesi ve $X$ serbest să̆ $R$-modül ise $Q \oplus X$ sağ $R$-modülünün semi-projektif olması için gerek ve yeter koşul $X^{\prime}$ den $Q^{\prime}$ ya bir $R$-epimorfizmasının olmamasıdır. Diğeri; eğer $R$ kesirler cismi $Q$ olan bir sağ Ore bölgesi ise $Q \oplus R$ sağ $R$-modülü semiprojektiftir. Ve sonuncusu da; eğer $R$ kesirler cismi $Q$ olan bir să̆ Ore bölgesi ve $X$ sonlu üreteçli projektif bir să̆ $R$-modül ise ve eğer $R$ sağ Noether yada sol Ore bölgesi ise o zaman $Q \oplus X$ semi-projektiftir. Bu sonuçlar sayesinde bir sonraki alt bölümde bir çok örnek elde edilmiştir.

Üçüncü alt bölümde, ikinci altbölümden çıkarılabilen genel örnekler yer almaktadır. Eğer $R$ kesirler cismi $Q$ olan bir temel ideal bölgesi ise ve $X, Q^{\prime}$ nun $R$ ' yi kapsayan bir özaltmodülü ise $M$ sonlu üreteçlidir ancak ve ancak $M$ projektiftir ancak ve ancak $M$ semi-projektiftir ancak ve ancak $M$ direk projektiftir.

$\mathrm{Bu}$ bölümün dördüncü alt bölümünde bir $M$ sağ $R$-modülünün semi-projektif örtüsü tanımlanmıştır, ayrıca yarıtam ve tam halkaların semi-projektif modüller ve semi-projektif örtüler kullanılarak bazı karakterizasyonları verilmiştir. Bu karakterizasyonların tamamı [3], [11], [14], [16], [17], [35], [57] and [59] nolu çalışmaların semi-projektif modüllere uyarlanmasıyla elde edilmiştir.

Beşinci alt bölümde ise yarıkalıtsal, kalıtsal ve yarıbasit halkalar semi-projektif modüller yardımıyla karakterize edilmiştir. Ve semi-projektif bir modülün her altmodülünün semi-projektif olmadığına dair bir örnek verilmiştir. Daha sonra ise her altmodülü semi-projektif olan semi-projektif să̆ $R$-modüller için $R$ halkaları incelenmiştir.

$M$ bir sağ $R$-modül, $N, M^{\prime}$ nin bir altmodülü ve $S=\operatorname{End}(M)$ olsun. [34] nolu çalışmada, Keskin-Tütüncü ve Tribak $S^{\prime}$ nin să̆ ideali olan $\operatorname{Hom}(M, N)^{\prime}$ yi $D(N)=$ 
$\{\varphi \in S \mid \operatorname{Im} \varphi \subseteq N\}$ ile göstermişlerdir ve eğer $M^{\prime}$ nin her $N$ altmodülü için $D(N)=\epsilon S$ olacak şekilde $S^{\prime}$ de bir $\epsilon$ idempotenti varsa $M$ modülünü dual Baer olarak adlandırmışlardır.

$M^{\prime}$ nin sıfırdan farklı bir endomorfizması $\alpha: M \longrightarrow M$ alınsın. Yukarıdaki tanım yardımıyla, 2.1 numaralı alt bölümde $S^{\prime}$ nin bir sağ ideali aşağıdaki gibi tanımlanmış ve bu idealin bazı özellikleri incelenmiştir ;

$$
D(\alpha)=\{\varphi \in S \mid \operatorname{Im} \varphi \subseteq \operatorname{Im} \alpha\}
$$

Tezin üçüncü bölümünde $M^{\prime}$ nin sıfırdan farklı $\alpha$ endomorfizması için $D(\alpha)$ ideali yardımıyla, quasi-projektif modüllerin başka bir genellemesi olarak, SGQ-projektif modüller tanımlanmış ve özellikleriyle birlikte incelenmiştir.

$M^{\prime}$ nin sıfırdan farklı her $s \in S$ endomorfizması için $S^{\prime} \operatorname{nin} D(s)=s S \oplus X$ koşulunu sağlayacak bir $X$ ideali varsa, $M^{\prime}$ ye $S G Q$-projektif modül denir. İkinci bölümün ilk alt bölümünde SGQ-projektif modülün genel özellikleri incelenmiştir. Her semi-projektif modülün bir SGQ-projektif modül olduğu, her SGQ-projektif modülün bir semi-Hopfian modül olduğu, her quasi-discrete SGQ-projektif modülün bir discrete modül olduğu ispatlanmıştır. Ayrıca SGQ-projektif modüller yardımıyla yarıbasit halkalar karakterize edilmiştir. Son olarak ise bir SGQ-projektif modülün her dik toplananının yine bir SGQ-projektif modül olduğu fakat dik toplamlarının bir SGQ-projektif modül olmadığı örnekle gösterilmiştir.

Bu bölümün ikinci altbölümünde ise SGQ-projektif modüllerin endomorfizmalar halkasının özellikleri incelenmiştir. Ĕğer $M$ bir zayıf tümlenmiş, $S G Q$-projektif, $\pi$ projektif modül ise endomorfizmalar halkası düzenlidir ancak ve ancak $\nabla=0$ ' dır. (Burada $\nabla M^{\prime}$ nin endomorfizmalar halkasının, görüntüleri $M^{\prime}$ de dar(small) olan endomorfizmalarını içeren bir să̆ idealidir).

Dördüncü bölümde öncelikle epi projektiflik, epi $\mathcal{K}$-projektiflik, epi $\mathcal{K}^{*}$-projektiflik kavramları ve aralarındaki bağlantılar incelenmiştir. Daha sonra yarıbasit halkalar quasi-epi $\mathcal{K}$-projektif, quasi-epi $\mathcal{K}^{*}$-projektif modüller yardımıla karakterize edilmiştir. Son olarak ise quasi(-epi) $\mathcal{K}^{*}$-projektif modüller ile Hopfian modüller arasındaki ilişki çalışılmıştır.

Son bölüm, Bölüm 5, formal üçgensel matris halkaları (bu bölümde bu tip halkalar T ile gösterilmiştir) üzerine kurulmuş modüllerle ilgilidir. Öncelikle bölümün 
başında formal üçgensel matris halkaları üzerine kurulu modül yapıları, bu modüllerin altmodül ve bölüm modülü yapıları [24] nolu makaleden faydalanılarak ayrıntılı bir biçimde anlatılmıştır. Yine [24] numaralı makalede formal üçgensel matris halkaları üzerine kurulmuş uniform, hollow, sonlu gömülmüş, projektif, üreteç veya proüreteç modüller karakterize edilmiş, $(X \oplus Y)_{T}{ }^{\prime} \operatorname{nin} \operatorname{Jacobson} \operatorname{radicali} \operatorname{Rad}(X \oplus Y)_{T}$ ve sokulu Soc $(X \oplus Y)_{T}$ tanımlanmıştır. Ayrıca [19]' da $T$ üzerindeki projektif sağ idealler tamamen karakterize edilmiştir. Bu tezin son bölümü $T$ halkası üzerine kurulu lifting modüller ve bir modüle göre projektiflik ile ilgilidir.

Anahtar Kelimeler: Ore bölgesi, semi-projektif modül, semi-projektif örtü, SGQprojektif modül, epi $\mathcal{K}$-projektif modül, formal üçgensel matris halkası. 


\section{ACKNOWLEDGEMENT}

I would like to express my special appreciation and thanks to my supervisor Professor Derya Keskin Tütüncü for her expert guidance, support, understanding and encouragement throughout my study and research.

I would also like to thank my committee members, Professor A. Çiğdem Özcan, Professor Evrim Akalan, Professor Nil Orhan Ertaş and Professor Fevzi Noyan Er for their help, comments and suggestions.

I wish to thank Professor Patrick F. Smith for his helpful advices and his contributions to my thesis.

I would also like to thank my friends Professor Pınar Aydoğdu, Professor Lale Özkahya and Professor Zümra Kavafoğlu for their advices, encouragement and friendship.

I wish to express my deep gratitude to my parents for their support and their positive energy. I also thank my brother Ediz Kuru for his big love and support. I would also like to thank my mother-in-law, father-in-law and sister-in-law Tuğba for their support.

At the end, I would like to express my appreciation to my beloved husband Hüseyin Kaleboğaz who has supported me throughout this process and has constantly encouraged me. I wouldn't have been able to complete this thesis without his continuous love, understanding and encouragement.

I would also like to acknowledge the supports "2214-A International Research Fellowship Programme" that I have received from The Scientific and Technological Research Council of Turkey (TÜBİTAK) from September 2013 to March 2014. 


\section{CONTENTS}

$\underline{\text { Page }}$

ABSTRACT

ÖZET

ACKNOWLEDGEMENT viii

TABLE OF CONTENTS ix

NOTATIONS X xi

1 PRELIMINARIES 1

1.1 Basic Notions . . . . . . . . . . . . . . . . . . . . . . 1

1.2 Projectivity Conditions of a Module . . . . . . . . . . . . 5

1.3 Rings of Fractions . . . . . . . . . . . . . . . . . . . . . . . 10

1.4 Division Rings of Fractions . . . . . . . . . . . . . . . . . . . 12

1.5 Goldie's Theorem . . . . . . . . . . . . . . . . . . . . . 13

1.6 Torsion and Torsion-free Modules . . . . . . . . . . . . . . . . . 15

2 SEMI-PROJECTIVE MODULES 18

2.1 Some Properties of Semi-Projective Modules . . . . . . . . . . . . . . 20

2.2 Direct Sums of Semi-Projective Modules Over Ore Domains . . . . . . . . . . . . . . . . . 31

2.3 Some Examples . . . . . . . . . . . . . . . . . . . 36

2.4 Semi-Projective Covers . . . . . . . . . . . . . . . . . . . . . . . 40

2.5 Rings Over Which Submodules of Semi-projective Right R-modules are Semi-projective . . . . . . . . . . . . . . . . . 44

3 SGQ-PROJECTIVE MODULES 52

3.1 Some Properties of SGQ-projective Modules . . . . . . . . . . . . . . 52

3.2 The Endomorphism Ring of SGQ-projective Modules . . . . . . . . . 57 
4 A STUDY ON $\mathcal{K}$-PROJECTIVITY AND $\mathcal{K}^{*}$-PROJECTIVITY

4.1 Properties of epi-K $\mathcal{K}\left(\mathcal{K}^{*}\right)$-projectivity . . . . . . . . . . . 63

4.2 Connections Between the Quasi(-epi) $\mathcal{K}\left(\mathcal{K}^{*}\right)$-projective Modules and the Hopfian Modules . . . . . . . . . . . . . 66

5 MODULES OVER FORMAL TRIANGULAR MATRIX RINGS 69 5.1 Lifting Modules Over $T \ldots \ldots$. . . . . . . . . . . . 71

5.2 Relative Projectivity of Modules Over T . . . . . . . . . . . 72

$\begin{array}{lr}\text { REFERENCES } & 78\end{array}$

$\begin{array}{lr}\text { INDEX } & 84\end{array}$

$\begin{array}{ll}\text { CURRICULUM VITAE } & 86\end{array}$ 


\section{NOTATIONS}

$R$

$M_{R}$

$\operatorname{Mod}-R$

$\bigoplus M_{i}$

$\prod M_{i}$

$M^{(\Lambda)}$

$M^{\Lambda}$

$\operatorname{ann} n_{R}^{r}(m)$

$A \leq M$

$A \leq_{e} M$

$A \leq_{d} M$

$A \ll M$

$\operatorname{Rad}(M)$

$J=J(R)$

$\operatorname{Soc}(M)$

$Z(M)$

$\operatorname{Hom}_{R}(M, N)$

$\operatorname{End}_{R}(M)$

$\operatorname{Ker} f$

$\operatorname{Im} f$

$\mathbb{Z}$

$\mathbb{Q}$

$\mathbb{N}$

$\mathbb{Z}_{n}$

$\mathbb{Z}_{p^{\infty}}$

$R_{n}$

PID
A noncommutative and associative ring with identity

A unitary right $R$-module

The category of right $R$-modules

Direct sum of $R$-modules $M_{i}$

Direct product of $R$-modules $M_{i}$

$\bigoplus_{(i \in \Lambda)} M_{i}, M_{i}=M$

$\prod_{(i \in \Lambda)} M_{i}, M_{i}=M$

$\{r \in R \mid m r=0\}$, the right annihilator of $m \in M$ in $R$

A submodule $A$ of an $R$-module $M$

An essential submodule $A$ of an $R$-module $M$

A direct summand $A$ of an $R$-module $M$

A small submodule $A$ of an $R$-module $M$

Jacobson radical of an $R$-module $M$

Jacobson radical of a ring $R$

$\cap\left\{N \mid N \leq_{e} M\right\}=\oplus\{L \mid L$ is a simple submodule of $M\}$

$\left\{m \in M \mid r_{R}(m) \leq_{e} R\right\}$

The set of $R$-module homomorphisms from $M$ to $N$

The set of $R$-endomorphisms of $M$

Kernel of an $R$-homomorphism $f$

Image of an R-homomorphism $f$

The set of integers

The set of rational numbers

The set of natural numbers

The cyclic group $\mathbb{Z} / n \mathbb{Z}$

Prüfer p-group

The set of $n \times n$ matrices over a ring $R$

Principal Ideal Domain 


\section{PRELIMINARIES}

In this chapter, we will give some basic notions and results which are frequently used in the following sections. Throughout this dissertation, $\mathrm{R}$ will denote an associative ring with identity and modules will be unitary right $R$-modules unless otherwise stated.

\subsection{Basic Notions}

Definitions 1.1.1 ([2]) A submodule $N$ of an $R$-module $M$ is called essential in $M$ or $M$ is called an essential extension of $N$ if every nonzero submodule of $M$ intersects $N$ nontrivially, i.e. for every submodule $L \leq M, N \cap L=0$ implies $L=0$. It is denoted by $N \leq_{e} M$. If every nonzero submodule of an $R$-module is essential in $M$, i.e., the intersection of any two nonzero submodules is nonzero then we say that $M$ is uniform.

Proposition 1.1.2 ([2]) Let $M$ be an $R$-module with submodules $K \leq N \leq M$ and $H \leq M$. Then the following hold:

1. $K \leq_{e} M$ if and only if for each $0 \neq x \in M$, there exists an $r \in R$ such that $0 \neq x r \in K$.

2. $K \leq_{e} M$ if and only if $K \leq_{e} N$ and $N \leq_{e} M$.

3. $H \cap K \leq_{e} M$ if and only if $H \leq_{e} M$ and $K \leq_{e} M$.

4. If $K \leq_{e} M$ and $f: L \longrightarrow M$ a homomorphism, then $f^{-1}(K) \leq_{e} L$.

5. Let $K_{1} \leq M_{1} \leq M, K_{2} \leq M_{2} \leq M$ and $M=M_{1} \oplus M_{2}$. Then $K_{1} \oplus K_{2} \leq_{e} M$ if and only if $K_{1} \leq_{e} M_{1}$ and $K_{2} \leq_{e} M_{2}$.

The following definition dualizes the notion of an essential module;

Definition 1.1.3 ([2]) A submodule $N$ of an $R$-module $M$ is called a small submodule of $M$ provided $K+N$ is a proper submodule of $M$ whenever $K$ is a proper submodule of $M$, i.e. for every submodule $L \leq M, K+L=M$ implies $L=M$. It is denoted by $N \ll M$. If every proper submodule of $M$ is small, then $M$ is called a hollow module. 
Proposition 1.1.4 ([2]) Let $M$ be an $R$-module. Then the following statements hold for $K \leq N \leq M$ and $H \leq M$ :

1. $N \ll M$ if and only if $K \ll M$ and $N / K \ll M / K$.

2. $H+K \ll M$ if and only if $H \ll M$ and $K \ll M$.

3. If $K \ll M$ and $f: M \longrightarrow L$ is an $R$-homomorphism, then $f(K) \ll L$. In particular, if $K \ll M \leq L$, then $K \ll L$.

4. Let $K_{1} \leq M_{1} \leq M, K_{2} \leq M_{2} \leq M$ and $M=M_{1} \oplus M_{2}$. Then $K_{1} \oplus K_{2} \ll M$ if and only if $K_{1} \ll M_{1}$ and $K_{2} \ll M_{2}$.

5. Let $K \leq L \leq M$. If $K \ll M$ and $L \leq_{d} M$, then $K \ll L$. In particular, if $K \ll M$ and $K \leq_{d} M$, then $K=0$.

The following definitions are taken from [2];

A simple module is a nonzero module $M$ in which the only submodules are 0 and $M$. An $R$-module $M$ is called semisimple if it is the direct sum of its simple submodules. If $R_{R}$ is semisimple, then $R$ is called a (right) semisimple ring. $R_{R}$ is semisimple if and only if ${ }_{R} R$ is semisimple. If $M$ is an $R$-module, then the socle of $M$, denoted by $\operatorname{Soc}(M)$, is the sum of all simple submodules of $M$ and therefore it is the largest semisimple submodule of $M$. It is equal to the intersection of all essential submodules. $\operatorname{Soc}(M)=M$ if and only if $M$ is semisimple. If a module $M$ does not contain any simple submodule, then we take $\operatorname{Soc}(M)=0$. The dual concept of socle is radical. The Jacobson radical $\operatorname{Rad}(M)$ of an $R$-module $M$ is the intersection of all maximal submodules of $M$, or equivalently is the sum of all small submodules of $M$. The Jacobson radical of a ring $R$ is denoted by $J(R)$, and it is an ideal of $R$. A nonzero $R$-module $M$ is said to be indecomposable if it is not a direct sum of two nonzero submodules; and $M$ is called local if it has a largest proper submodule $($ namely $\operatorname{Rad}(M))$. A local module is indecomposable.

Proposition 1.1.5 ([2]) $A$ ring $R$ is semisimple if and only if every right $R$-module is semisimple. 
Proposition 1.1.6 ([2]) For a ring $R$ with radical $J(R)$ the following statements are equivalent;

1. $R / J(R)$ is semisimple

2. $R / J(R)$ is right artinian

3. Every product of simple right $R$-modules is semisimple

4. Every product of semisimple right $R$-modules is semisimple

5. For every right $R$-module $M, \operatorname{Soc}(M)=r_{M}(J(R))=\{m \in M \mid J(R) m=0\}$.

The following definitions are from [2] and [19];

The set $\operatorname{ann}_{R}(M)=\{r \in R \mid M r=0\}$ is the annihilator of right $R$-module $M$ in R. $M$ is called faithful if $\operatorname{ann}_{R}(M)=0$. For any $m \in M$, the set $a n n_{R}^{r}(m)=\{r \in$ $R \mid m r=0\}$ is the right annihilator of $m$ in $R$, and it is a right ideal of $R$.

A prime ideal in a ring $R$ is any proper ideal $P$ of $R$ such that, whenever $I$ and $J$ are ideals of $R$ with $I J \subseteq P$, either $I \subseteq P$ or $J \subseteq P$. A prime ring is a ring in which 0 is a prime ideal. An ideal $P$ in a ring $R$ is right primitive provided $P=\operatorname{ann}_{R}(A)$ for some simple right $R$-module $A$. A right primitive ring is any ring in which 0 is a right primitive ideal, i.e., any ring which has a faithful simple right module.

A semiprime ideal in a ring $R$ is any ideal of $R$ which is an intersection of prime ideals. A semiprime ring is any ring in which 0 is a semiprime ideal.

Definitions 1.1.7 ([19]) For a right $R$-module $M$, the singular submodule of $M$ is the set

$$
Z(M)=\{m \in M \mid m I=0 \text { for some essential right ideal } \mathrm{I} \text { of } \mathrm{R}\} .
$$

An $R$-module $M$ is called a singular module provided $Z(M)=M$ and it is called a nonsingular module provided $Z(M)=0$.

Let $M$ be an $R$-module and $N \leq M . M / N$ is singular whenever $N \leq_{e} M$. The converse holds if $M$ is nonsingular. The class of all nonsingular $R$-modules is closed 
under submodules, direct products, essential extensions and module extensions. The class of all singular $R$-modules is closed under submodules, factor modules and direct sums, ([19]).

Proposition 1.1.8 ([19]) If every principal right ideal of $R$ is projective then $R$ is nonsingular.

Definition 1.1.9 ([2]) A two sided ideal $I$ of a $\operatorname{ring} R$ is called right $T$-nilpotent if for every sequence $\left\{x_{i} \mid i \geq 1\right\}$ in $I$, there exists an integer $n \geq 1$ such that $x_{n} \ldots x_{2} x_{1}=0$.

Lemma 1.1.10 ([18]) Let $I$ be a right $T$-nilpotent two sided ideal of a ring $R$. Then for every right $R$-module $M, M I$ is small in $M$.

Definition 1.1.11 ([2]) Let $\mathcal{U}$ be a non-empty set (class) of objects in a category $\mathcal{C}$. An object $A$ in $\mathcal{C}$ is said to be generated by $\mathcal{U}$ or $\mathcal{U}$-generated if, for every pair of distinct morphisms $f, g: A \longrightarrow B$ in $\mathcal{C}$, there is a morphism $h: U \longrightarrow A$ with $U \in \mathcal{U}$ and $h f \neq h g$. In case $\mathcal{U}$ consists of just one $U \in O b j(\mathcal{C})$, we call $U$ a generator for $A$.

Let $M$ be an $R$-module. A set $\mathcal{S}$ of submodules of $M$ satisfies the ascending chain condition (acc) in case for every chain $M_{1} \leq M_{2} \leq \ldots \leq M_{n} \leq \ldots$ in $\mathcal{S}$, there is an $n$ with $M_{n+i}=M_{n}(i=1,2, \ldots)$. Turn the inequalities around for the descending chain condition (dcc). A module $M$ is noetherian in case the lattice $\mathcal{S}(M)$ of all submodules of $M$ satisfies the acc. A module $M$ is artinian in case $\mathcal{S}(M)$ satisfies the dcc. A ring $R$ is called right noetherian (right artinian) if $R_{R}$ is noetherian (artinian). A similar definition may be made on the left. $R$ is noetherian (artinian) if it is both right and left noetherian (artinian). The artinian and noetherian properties are inherited by submodules and factor modules. Those definitions are from [2].

Proposition 1.1.12 ([2]) A right $R$-module $M$ is noetherian if and only if every submodule of $M$ is finitely generated.

Proposition 1.1.13 ([2]) $A$ ring $R$ is right noetherian (artinian) if and only if every finitely generated right $R$-module is noetherian (artinian). 


\subsection{Projectivity Conditions of a Module}

It is well-known that projectivity conditions on a module $M$ often allow us to relate properties of $M$ to properties of its endomorphism ring. In this section we review various forms of projectivity.

Definition 1.2.1 ([7]) An $R$-module $P$ is called $M$-projective (or $P$ is projective relative to $M$ ) if every diagram in $\operatorname{Mod}-R$ with exact row

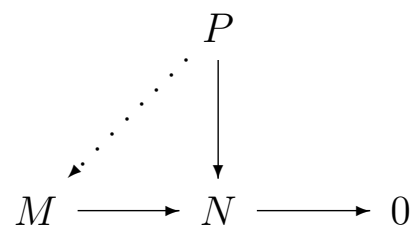

can be extended commutatively by some morphism $P \rightarrow M$, or, equivalently, the functor $\operatorname{Hom}(P,-)$ is exact with respect to all exact sequences of $R$-modules of the form

$$
0 \longrightarrow K \longrightarrow M \longrightarrow N \longrightarrow 0
$$

An $R$-module $P$ is called projective in case it is $M$-projective for every $R$-module $M$. A ring is a projective module over itself. Every free module is a projective module, $([2])$.

Theorem 1.2.2 ([54]) Assume $M \in \operatorname{Mod}-R$ and that $\left\{U_{\lambda}\right\}_{\Lambda}$ is a family of $R$ modules. The direct sum $\bigoplus_{\Lambda} U_{\lambda}$ is $M$-projective if and only if every $U_{\lambda}$ is $M$ projective. In particular $\bigoplus_{\Lambda} U_{\lambda}$ is projective if and only if every $U_{\lambda}$ is projective.

Proposition 1.2.3 ([54]) Let $P$ be an $R$-module. Then,

1. If $0 \longrightarrow M^{\prime} \longrightarrow M \longrightarrow M^{\prime \prime} \longrightarrow 0$ is an exact sequence in $M o d-R$ and $P$ is $M$-projective, then $P$ is $M^{\prime}$ - and $M^{\prime \prime}$-projective.

2. For any finite family $\left\{M_{i}\right\}_{i=1}^{n}$ of $R$-modules $P$ is $\bigoplus_{i=1}^{n} M_{i}$-projective if and only if $P$ is $M_{i}$-projective for $i=1,2, \ldots, n$.

3. If $P$ is finitely generated and $M_{\lambda}$-projective for any family $\left\{M_{\lambda}\right\}_{\Lambda}$ of $R$-modules, then $P$ is also $\bigoplus_{\Lambda} M_{\lambda}$-projective. 
Note that Proposition 1.2.3 (2) is not true for infinite families. For example, if $R=\mathbb{Z}$, then $\mathbb{Q}$ is $\mathbb{Z}$-projective but is not $\mathbb{Z}^{(\mathbb{N})}$-projective.

Definition 1.2.4 ([51]) A short exact sequence $0 \longrightarrow A \stackrel{i}{\longrightarrow} B \stackrel{p}{\longrightarrow} C \longrightarrow 0$ splits if there exists a map $j: C \longrightarrow B$ with $p j=1_{C}$.

Proposition 1.2.5 ([51]) If an exact sequence $0 \longrightarrow A \stackrel{i}{\longrightarrow} B \stackrel{p}{\longrightarrow} C \longrightarrow 0$ splits, then $B \cong A \oplus C$.

Proposition 1.2.6 ([2]) The following statements are equivalent for an $R$-module $P$ :

1. $P$ is projective.

2. Every epimorphism $M \longrightarrow P \longrightarrow 0$ splits.

3. $P$ is isomorphic to a direct summand of a free $R$-module.

Definitions 1.2.7 ([2]) A ring $R$ is called von Neumann regular if for every element $r \in R$ there exists $a \in R$ such that $r=\operatorname{rar}$. If $R$ is a von Neumann regular ring then every principal right ideal is direct summand.

Definition 1.2.8 ([2]) A projective $R$-module $P$ is a projective cover of $M$ if there exists an epimorphism $\varphi: P \longrightarrow M$ with small kernel, i.e. $\operatorname{Ker} \varphi \ll P$.

A module may not have a projective cover. Every projective R-module is a projective cover of itself. Note that the $\mathbb{Z}$-modules $\mathbb{Z}_{2}$ and $\mathbb{Q}$ have no projective covers.

Definitions 1.2.9 A ring $R$ is called right perfect if every right $R$-module has a projective cover. Perfect rings were characterized by H. Bass in [3]. A semiperfect ring is a ring over which every finitely generated right module has a projective cover. This property is left-right symmetric. Right perfect rings are semiperfect.

Theorem 1.2.10 ([2]) Let $R$ be a ring with $J(R)$. Then the following statements are equivalent;

1. $R$ is right perfect, 
2. $R / J(R)$ is semisimple and $J(R)$ is right T-nilpotent,

3. Every flat right $R$-module is projective.

4. $R$ satisfies the descending chain condition for cyclic ideals.

Definitions 1.2.11 ([54]) A ring $R$ is called right (semi)hereditary if every (finitely generated) right ideal of $R$ is projective. Equivalently, $R$ is right (semi)hereditary if and only if every (finitely generated) submodule of a projective right $R$-module is projective. $R$ is called a right PP-ring if and only if every principal right ideal of $R$ is projective. It is clear that every right hereditary ring is right PP-ring. From the Proposition 1.1.8 every PP-ring (so hereditary ring) is nonsingular.

Theorem 1.2.12 ([58]) $R$ is right hereditary if and only if every submodule of a projective $R$-module is direct projective.

Theorem 1.2.13 ([28]) Let $R_{n}$ be an $n \times n$ matrix ring with entries from $R . R$ is right (semi)hereditary if and only if $R_{n}$ is (semi)hereditary. $R$ is right (semi)perfect if and only if $R_{n}$ is (semi)perfect.

Definition 1.2.14 ([54]) An $M$-projective module $M$ is called quasi-projective (or self-projective).

Proposition 1.2.15 ([12]) The direct sum $M_{1} \oplus M_{2}$ is quasi-projective if and only if $M_{i}$ is $M_{j}$-projective for $i, j \in\{1,2\}$.

Proposition 1.2.16 ([7]) Let $M$ be quasi-projective and $U \leq M$ :

1. If $U$ is fully invariant, then $M / U$ is quasi-projective.

2. If $U \ll M$ and $M / U$ is quasi-projective, then $U$ is fully invariant in $M$.

Corollary 1.2.17 ([50]) Let I be a two sided ideal of a ring $R$. Then $R / I$ is quasiprojective as an $R$-module.

Proposition 1.2.18 ([17]) Let $M$ be a right $R$-module and $I$ a two sided ideal of $R$ contained in the annihilator of $M$. Then $M$ is quasi-projective over $R$ if and only if it is quasi-projective over $R / I$. 
Theorem 1.2.19 ([12]) An abelian group $A$ is quasi-projective if and only if it is free or a torsion group such that every p-component $A_{p}$ is a direct sum of cyclic groups of the same order.

Theorem 1.2.20 ([1]) Let $R$ be a Dedekind domain and $M$ be a torsion $R$-module (see, Definition 1.6.2). $M$ is quasi-projective if and only if for each maximal (prime) ideal $P$ in $R$, the P-primary component of $M$ is quasi-projective.

Definition 1.2.21 ([7]) An $R$-module $M$ is called $\pi$-projective if, for any two submodules $U, V \leq M$ with $U+V=M$, the following equivalent conditions are satisfied:

1. There exists $f \in \operatorname{End}(M)$ with $\operatorname{Im}(f) \subseteq U$ and $\operatorname{Im}(1-f) \subseteq V$;

2. The canonical epimorphism $U \oplus V \rightarrow M,(u, v) \mapsto u+v$, splits;

3. $\operatorname{End}(M)=\operatorname{Hom}(M, U)+\operatorname{Hom}(M, V)$

Definition 1.2.22 ([55]) An $R$-module $M$ is said to be intrinsically projective if every diagram with exact row

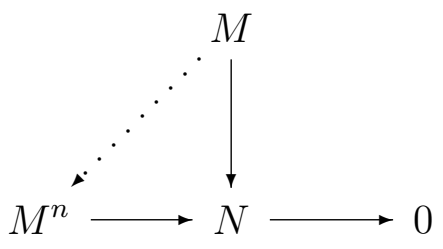

where $n \in \mathbb{N}$ and $N \leq M$, can be extended commutatively by some $M \longrightarrow M^{n}$.

$M$ is called semi-projective if the above condition (only) holds for $n=1$. It can easily be seen that, $M$ is semi-projective if and only if for every cyclic right ideal $I$ of $\operatorname{End}\left(M_{R}\right), I=\operatorname{Hom}_{R}(M, M I),([55])$.

Of course every quasi-projective module is intrinsically projective. However there are also other types of examples:

Examples 1.2.23 ([55]) Let $M$ be an $R$-module with $S=\operatorname{End}\left(M_{R}\right)$.

1. If kernels of endomorphisms of $M$ are $M$-generated and $S$ is a right PP-ring, then $M$ is semi-projective.

2. If $S$ is a von Neumann regular ring, then $M$ is intrinsically projective. 
Definitions 1.2.24 ([7]) Let $M$ be a nonzero right $R$-module. A submodule $N \leq M$ is called a weak supplement of a submodule $L$ of $M$ if $N+L=M$ and $N \cap L \ll M$. The module $M$ is called weakly supplemented if every submodule $N$ of $M$ has a weak supplement.

For submodules $N, L \leq M$, the following are equivalent:

1. $N$ is minimal in the set of submodules $\{K \leq M \mid L+K=M\}$.

2. $L+N=M$ and $L \cap N \ll N$.

If one of these conditions holds, then $N$ is called a supplement of $L$ in $M$. A submodule $N$ of $M$ is said to have ample supplements in $M$ if, for every $L \subset M$ with $N+L=M$, there is a supplement $L^{\prime}$ of $N$ with $L^{\prime} \subset L$. The module $M$ is called supplemented if any submodule has a supplement, finitely supplemented if finitely generated submodules have supplements, amply supplemented if all submodules have ample supplements in $M$. Every amply supplemented module is supplemented.

Given submodules $K \subset L \subset M$, the inclusion $K \subset L$ is said to be cosmall in $M$ if $L / K \ll M / K$. A submodule $L \subset M$ is said to be coclosed in $M$, if $L$ has no proper submodule $K$ for which $K \subset L$ is cosmall in $M$. Thus $L$ is coclosed in $M$ if and only if for any proper submodule $K \subset L$, there is a submodule $N$ of $M$ such that $L+N=M$ but $K+N \neq M$. Obviously, any direct summand $L$ of $M$ is coclosed in $M$.

Proposition 1.2.25 ([7]) Let $K \subseteq L \subseteq M$ be submodules. If $K$ is coclosed in $M$, then $K$ is coclosed in $L$. The converse is true if $L$ is coclosed in $M$.

Consider the following conditions for an $R$-module $M$ :

$\left(D_{1}\right) \quad M$ is amply supplemented and every coclosed submodule of $M$ is a direct summand of $M$.

$\left(D_{2}\right)$ If $M / N$ is isomorphic to a direct summand of $M$, then $N$ is a direct summand of $M$.

$\left(D_{3}\right)$ If $M_{1}$ and $M_{2}$ are direct summands of $M$ with $M=M_{1}+M_{2}$, then $M_{1} \cap M_{2}$ is a direct summand of $M$. 
An $R$-module $M$ is called lifting if $M$ satisfies $\left(D_{1}\right) . M$ is called discrete if it is lifting and satisfies $\left(D_{2}\right)$ and quasi-discrete if it is lifting and satisfies $\left(D_{3}\right)$. Every discrete module is quasi-discrete, (see [45]).

Lemma 1.2.26 ([45]) Let $M$ be a module with (D2). If $M_{1}, M_{2} \leq_{d} M$, then any epimorphism $M_{1} \longrightarrow M_{2}$ splits.

As a dual version of projectivity, injective modules are defined as follows: A right module $A$ over a ring $R$ is injective provided that, for any right $R$-module $B$ and any submodule $C$ of $B$, all homomorphisms $C \rightarrow A$ extend to homomorphisms $B \rightarrow A$. An injective hull (or injective envelope) for a module $A$ is any injective module which is an essential extension of $A$. The notation $E(A)$ is used for an injective hull of $A$. A module $A$ has finite rank provided $E(A)$ is a finite direct sum of indecomposable submodules. Goldie proved that a module $A$ has finite rank if and only if $A$ contains no infinite direct sums of nonzero submodules. ([20])

\subsection{Rings of Fractions}

In this section we will introduce how a ring of fractions can be constructed. This subsection is from [20].

Definition 1.3.1 ([20]) A regular element in a ring $R$ is any nonzero-divisor, namely, any element $x \in R$ such that $\operatorname{ann}_{R}^{r}(x)=0$ and $\operatorname{ann}_{R}^{l}(x)=0$.

Let $R$ be a ring and $X$ a set of regular elements in $R$; we seek to build a ring whose elements are fractions with numerators from $R$ and denominators from $X$. In the commutative case, the elementary notation $\frac{r}{x}$ is convenient and familiar, but in the noncommutative case we must be more careful. When we divide by $x$ (multiply by $x^{-1}$ ), we must decide whether to place the denominator on the right or the left of the numerator, i.e., whether we shall work with $r x^{-1}$ or $x^{-1} r$. Thus, we have two possible rings of fractions, one with right-hand denominators and one with left-hand denominators.

Definition 1.3.2 ([20]) A multiplicative set in a $\operatorname{ring} R$ is a subset $X \subseteq R$ such that $1 \in X$ and $X$ is closed under multiplication. 
Definition 1.3.3 ([20]) Let $X$ be a multiplicative set in a ring $R$. Then $X$ satisfies the right Ore condition provided that for each $x \in X$ and $r \in R$, there exist $y \in X$ and $s \in R$ such that $r y=x s$, that is, $r X \cap x R \neq \emptyset$. A multiplicative set satisfying the right Ore condition is called a right Ore set for short.

Any multiplicative set in a commutative ring is an Ore set.

Lemma 1.3.4 ([20]) Let $X$ be a right Ore set in a ring $R$. For given any elements $x_{1}, \ldots, x_{n} \in X$, there exist $s_{1}, \ldots, s_{n} \in R$ such that $x_{1} s_{1}=\cdots=x_{n} s_{n}$ and $x_{1} s_{1} \in X$ , that is, $x_{1} R \cap \cdots \cap x_{n} R \cap X \neq \emptyset$.

Definition 1.3.5 ([20]) Let $R$ be a ring and $X \subseteq R$ a multiplicative set of regular elements in $R$. A right ring of fractions (right quotient ring) for $R$ with respect to $X$ is any overring $S \supseteq R$ such that:

1. Every element of $X$ is invertible in $S$.

2. Every element of $S$ can be expressed in the form $a x^{-1}$ for some $a \in R$ and $x \in X$.

Left rings of fractions are defined analogously. Note that here we have a necessary condition for the existence of ring of fractions in general case (in commutative and non-commutative): Given $b \in R$ and $x \in X$, there must exist $c \in R$ and $z \in X$ such that $x^{-1} b=c z^{-1}$, that is, $b z=x c$. This is precisely the right Ore condition that we met above.

Lemma 1.3.6 ([20]) Let $R$ be a ring and $X$ a multiplicative set of regular elements in $R$, and assume that there exists a right ring of fractions, say $S$, for $R$ with respect to $X$.

1. $X$ is a right Ore set in $R$.

2. Given any $s_{1}, \ldots, s_{n} \in S$, there exists $a_{1}, \ldots, a_{n} \in R$ and $x \in X$ such that $s_{i}=a_{i} x^{-1}$.

3. Let $a, b \in R x, y \in X$. Then $a x^{-1}=b y^{-1}$ in $S$ if and only if there exists $c, d \in R$ such that $a c=b d$ and $x c=y d \in X$. 
The discussion above gives all the clues to construct rings of fractions. This construction was developed by Ore and Asano in the 1930s and 1940s. Let $X$ be a right Ore set of regular elements in a ring $R$. The construction can be summarized in the following five steps.

1. Define a relation $\sim$ on $R \times X$ as follows: $(a, x) \sim(b, y)$ if and only if there exist $c, d \in R$ such that $a c=b d$ and $x c=y d \in X$. Then $\sim$ is an equivalence relation. Let $[a, x]$ denote the $\sim$-equivalence class of any pair $(a, x)$ in $R \times X$, and let $S$ denote the set of these equivalence classes.

2. Given $[a, x]$ and $[b, y]$ in $S$, choose $c, d \in R$ such that $x c=y d \in X$, and set $[a, x]+[b, y]=[a c+b d, x c]$. Then + is a well-defined operation on $S$.

3. Given $[a, x]$ and $[b, y]$ in $S$, choose $c \in R$ and $z \in X$ such that $b z=x c$, and set $[a, x] \cdot[b, y]=[a c, y z]$. Then $\cdot$ is a well-defined operation on $S$.

4. $(S,+, \cdot)$ is a ring.

5. The rule $r \mapsto[r, 1]$ defines an isomorphism of $R$ onto a subring of $S$, and when $R$ is identified with this subring, $S$ becomes a right ring of fractions for $R$ with respect to $X$.

Now we will describe a ring of fractions as an $R$-module. Let $X$ be a right Ore set of regular elements in a ring $R$, and suppose that there exist a right ring of fractions for $R$ with respect to $X$, say it $S . S$ is determined as a right $R$-module:

$$
S_{R}=\left\{s \in E\left(R_{R}\right) \mid s x \in R \text { for some } x \in X\right\}
$$

Theorem 1.3.7 ([20]) Let $R$ be a ring and $X \subseteq R$ a multiplicative set of regular elements. Then there exists a right ring of fractions for $R$ with respect to $X$ if and only if $X$ is a right Ore set.

\subsection{Division Rings of Fractions}

The definitions and the results in this subsection are from [20]. 
Definition 1.4.1 ([20]) A classical right quotient ring for a $\operatorname{ring} R$ is a right ring of fractions for $R$ with respect to the set of all regular elements in $R$. If $R$ has a classical right quotient ring $Q$, it is also said that $R$ is a right order in $Q$.

By Theorem 1.3.7, $R$ has a classical right quotient ring if and only if the set of regular elements in $R$ is a right Ore set. For example, every commutative ring has a classical quotient ring. In the case of commutative domain, the classical quotient ring is its quotient field. A non-commutative domain need not have a classical quotient ring, but if one exists, it will be a division ring.

Definition 1.4.2 ([20]) A right Ore domain is any domain $R$ in which the nonzero elements form a right Ore set, i.e., for each nonzero $x, y \in R$, there exists $r, s \in R$ such that $x r=y s \neq 0$.

Every commutative domain and also right noetherian domain is right Ore and if $R$ is a right Bezout domain (i.e., a domain in which every finitely generated right ideal is principal), $R$ is right Ore.

Theorem 1.4.3 ([20]) For a ring $R$ the following conditions are equivalent:

1. There exists a right Ore set $X$ of regular elements in $R$ such that $R X^{-1}$ is a division ring.

2. $R$ has a classical quotient ring which is a division ring.

3. $R$ is a right Ore domain.

If $R$ is a right Ore domain, its classical right quotient ring is usually called the right quotient division ring of $R$.

\subsection{Goldie's Theorem}

Goldie's Theorem, provides necessary and sufficient conditions for a ring to have a classical right quotient ring which is semisimple. In this subsection we give some definitions and results from [20] and [44]. 
Definition 1.5.1 ([20]) A right annihilator of a $\operatorname{ring} R$ is any right ideal of $R$ which equals the right annihilator of some subset of $R$.

Proposition 1.5.2 ([20]) Suppose that a ring $R$ has a right noetherian classical right quotient ring $Q$. Then $R_{R}$ has finite rank and $R$ has the acc (ascending chain condition) on right annihilators. Moreover, if $Q$ is semisimple, then $R$ must be semiprime.

Proposition 1.5.2 says that any ring which has a semisimple classical right quotient ring must be a semiprime right Goldie ring. The converse statement is the main content of Goldie's Theorem.

Definition 1.5.3 ([20]) A right Goldie ring is any $\operatorname{ring} R$ such that $R_{R}$ has finite rank and $R$ has the acc on right annihilators.

For example, every right noetherian ring is right Goldie.

Proposition 1.5.4 ([20]) Let $R$ be a semiprime right Goldie ring. For any $x \in R$, $x$ is a regular element if and only if ann $_{R}^{r}(x)=0$ if and only if $x R \leq_{e} R_{R}$.

A ring $R$ is right bounded if every essential right ideal of $R$ contains an ideal which is essential as a right ideal in $R$. A prime $\operatorname{ring} R$ is right bounded if and only if every essential right ideal of $R$ contains a nonzero ideal. A polynomial identity ring, or a PI-ring for short is defined as a ring all of whose elements satisfy some polynomial identity.

Proposition 1.5.5 ([44]) A prime PI-ring is a bounded Goldie ring.

Proposition 1.5.6 ([44]) Let $R$ be a semiprime PI-ring with centre $C$ and let $I$ be a nonzero ideal of $R$. Then there exists a nonzero element in $I \cap C$.

Theorem 1.5.7 ([44]) If $R$ is a primitive PI-ring of minimal degree $d$, then $R$ is a central simple algebra of dimension $(d / 2)^{2}$ over its center.

Theorem 1.5.8 ([20]) (Goldie's Regular Element Lemma) Let $R$ be a semiprime right Goldie ring and $I$ a right ideal of $R$. Then $I$ is an essential right ideal if and only if I contains a regular element. 
Corollary 1.5.9 ([20]) If $R$ is a prime right Goldie ring, then every nonzero ideal of $R$ contains a regular element.

Theorem 1.5.10 ([20]) (Goldie's Theorem) A ring $R$ has a semisimple classical right quotient ring if and only if $R$ is a semiprime right Goldie ring.

\subsection{Torsion and Torsion-free Modules}

All the definitions and the results in this subsection can be found in [20], [41], [13] and $[9]$.

Different kinds of 'torsion' already make appearances in the theory of abelian groups. For instance, if $A$ is an abelian group (written additively, as a $\mathbb{Z}$-module), then we have the torsion subgroup;

$$
T(A)=\{a \in A \mid m a=0 \text { for some nonzero } m \in \mathbb{Z}\}
$$

and, for each prime $\mathrm{p}$, the p-torsion (or p-primary) subgroup;

$$
T_{p}(A)=\left\{a \in A \mid p^{n} a=0 \text { for some } n \in \mathbb{N}\right\} .
$$

The common factor in these definitions is that each of the above subgroups has the form $\{a \in A \mid x a=0$ for some $x \in X\}$, where $X$ is a subset of $\mathbb{Z}$ which is closed under multiplication. Although some restrictions will be needed in transferring this idea to modules over a noncommutative ring, we can begin the discussion in complete generality.

Let $X$ be a multiplicatively closed set. Now let $A$ be an $R$-module. It is said that $A$ is $X$-torsion provided each element of $A$ is annihilated by some element of $X$, and that $A$ is $X$-torsion-free if the only element of $A$ annihilated by any element of $X$ is 0 . In particular, if $R=\mathbb{Z}$ and $X=\mathbb{Z}-\{0\}$, then $X$-torsion and $X$-torsion-free coincide with the notions of torsion and torsion-free for abelian groups, while if we take $X=\left\{p^{n} \mid n \in \mathbb{N}\right\}$ for a prime $p$, we get the concepts of $p$-torsion and $p$-torsionfree abelian groups. Up to now we did not define a general notion of $X$-torsion submodule for a right $R$-module $A$, namely, as the set $t_{X}(A)=\{a \in A \mid a x=$ 0 for some $x \in X\}$. The reason is that this set does not always produce submodules. 
Lemma 1.6.1 ([20]) Let $X$ be a right Ore set in a ring $R$. Then for any right $R$-module $A$, the set;

$$
t_{X}(A)=\{a \in A \mid a x=0 \text { for some } x \in X\}
$$

is a submodule of $A$.

Definition 1.6.2 ([20]) The torsion submodule of a right $R$-module $A$ is the set

$$
t(A)=\{a \in A \mid a x=0 \text { for some regular element } x \in R\}
$$

(this is a submodule of $A$ by Lemma 1.6.1), and we say that $A$ is torsion when $t(A)=A$ and torsion-free when $t(A)=0$.

Lemma 1.6.3 ([20]) Let $X$ be a right Ore set in a ring $R$. Then;

1. If $A$ is any right $R$-module, then $t_{X}(A)$ is an $X$-torsion module and $A / t_{X}(A)$ is an $X$-torsion-free module.

2. All submodules, factor modules, and sums (direct or not) of X-torsion right $R$-modules are $X$-torsion.

3. If $B \leq A$ are right $R$-modules with $B$ and $A / B$ both $X$-torsion, then $A$ is $X$-torsion.

4. All submodules and direct products of $X$-torsion-free right $R$-modules are $X$ torsion-free.

5. Let $B \leq A$ be right $R$-modules such that $B$ is $X$-torsion-free. If $B \leq_{e} A$, then $A$ is $X$-torsion-free.

6. If $B \leq A$ are right $R$-modules with $B$ and $A / B$ both $X$-torsion-free, then $A$ is $X$-torsion-free.

Definition 1.6.4 ([20]) A right module $A$ over a $\operatorname{ring} R$ is divisible provided $A x=$ $A$ for all regular elements $x \in R$. 
For example, every injective module is divisible. Over $\mathbb{Z}$, more generally, over any principal right ideal domain, the divisible right modules are exactly the injective right modules. Over other domains, however, divisible modules need not be injective. But over a commutative domain all torsion-free divisible modules are injective and the same result holds for semiprime Goldie rings, as follows.

Proposition 1.6.5 ([20]) (Gentile, Levy) Let A be a torsion-free right module over a semiprime right Goldie ring $R$. Then $A$ is divisible if and only if it is injective.

Proposition 1.6.6 ([41]) A ring $R$ is semiprime right Goldie if and only if $Z(M)=$ $t(M)$ for all right $R$-modules $M$.

Proposition 1.6.7 ([13]) Let $R$ be a domain. Then a torsion-free divisible $R$-module is a vector space over the quotient field $Q$ of $R$, i.e., it is a direct sum of copies of $Q$.

Proposition 1.6 .8 ([9, p.773]) A finitely generated module over a Dedekind domain is projective if and only if it is torsion-free. 


\section{SEMI-PROJECTIVE MODULES}

Semi-projective modules were first defined as a generalization of quasi-projective modules in 1991 by $\mathrm{R}$. Wisbauer in [54, p. 260]. An $R$-module $M$ is called semiprojective provided for all endomorphisms $\alpha$ and $\beta$ of $M$ with $\beta(M) \subseteq \alpha(M)$ there exists an endomorphism $\gamma$ of $M$ such that $\beta=\alpha \gamma$.



It is observed in [54, p. 260] that an $R$-module $M$ with endomorphism ring $S=\operatorname{End}\left(M_{R}\right)$ is semi-projective if and only if $\alpha S=\operatorname{Hom}_{R}(M, \alpha(M))$ for all $\alpha \in S$.

In 1996, in [55], R. Wisbauer defined a module $M$ to be intrinsically projective if every diagram with exact row

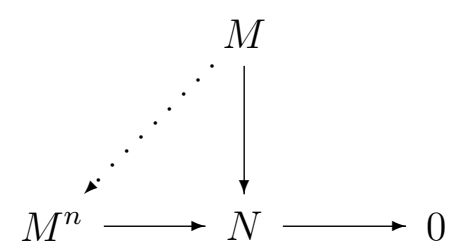

where $n \in \mathbb{N}$ and $N \leq M$, can be extended commutatively by some $M \longrightarrow M^{n}$. M is semi-projective if the above condition only holds for $n=1$. So being intrinsically projective is stronger than being semi-projective, and every quasi-projective module is intrinsically projective.

He gave in [54] that if $M$ is a finitely generated, semi-projective $R$-module satisfying dec (descending chain condition) for cyclic submodules, then $\operatorname{End}_{R}(M)$ satisfies dcc for cyclic left ideals. In [54], it is also given that if $M$ is an artinian and semiprojective module, then $S$ is semi-primary (i.e. $S / \operatorname{Jac}(S)$ is semisimple and $\operatorname{Jac}(S)$ is nilpotent).

In [55], he gave that $M_{R}$ is a module with endomorphism $\operatorname{ring} S$ is semi-projective if $S$ is a right $P P$-ring and kernels of endomorphisms of $M$ are $M$-generated.

In 2002, H. Tansee and S. Wongwai, in [52], defined $M$-principally projective modules as a dual version of $M$-principally injective modules. They called a right 
$R$-module $N M$-principally projective if every $R$-homomorphism from $N$ to an $M$ cyclic submodule of $M$ (If a submodule $K$ of $M$ is isomorphic to a factor module of $M$, then it is an $M$-cyclic submodule of $M$ ) can be lifted to an $R$-homomorphism from $N$ to $M$. In [52, Theorem 2.7] they proved that a module $M$ is $M$-principally projective if and only if it is semi-projective.

In 2007, A. Haghany and M. R. Vedadi investigated semi-projective retractable modules and endomorphism rings of such modules in [25].

In 2008, in [53] the 'relative' version of semi-projectivity was considered by X. Wang and J. Chen. They defined $N$ to be $M$-semi-projective if any homomorphism from $N$ to an $M$-cyclic submodule $f(M)$ of $N$ can be factored through a homomorphism from $N$ to $M$ and $f$, where $f \in \operatorname{Hom}(M, N)$. If $N=M$, then $M$ is semi-projective. This condition is equivalent to $M$-principally projectivity in [52]. They proved that if $N$ is $M$-semi-projective, then $N$ is $M / K$-semi-projective for any submodule $K$ of $M$ and if $N$ is $M$-semi-projective then for any direct summand $P$ of $N, P$ is $M$-semi-projective.

In 2011, V. Kumar, A. J. Gupta, B. M. Pandeya and M. K. Patel [40] studied $M$-SP-projective modules as a generalization of semi-projective modules.

This section concerns semi-projective modules and has four subsections. In the first subsection we give some basic properties of semi-projective modules and provide some characterizations. We prove that every nonsingular extending module is semiprojective. In general, for an $R$-module $M$ we have the following hierarchy:

$\mathrm{M}$ is quasi-projective $\Rightarrow \mathrm{M}$ is semi-projective $\Rightarrow \mathrm{M}$ is direct projective.

For the converse, we show that if $R$ is a Dedekind domain and if $M$ is an $R$-module which is a direct sum of cyclic submodules, then

$\mathrm{M}$ is quasi-projective $\Leftrightarrow \mathrm{M}$ is semi-projective $\Leftrightarrow \mathrm{M}$ is direct projective.

And in this chapter we also prove that if $R$ is a prime PI-ring and $M$ is a divisible $R$-module, then;

$\mathrm{M}$ is semi-projective $\Leftrightarrow \mathrm{M}$ is direct projective $\Leftrightarrow \mathrm{M}$ is semi-Hopfian $\Leftrightarrow \mathrm{M}$ is nonsingular.

It is shown that every direct summand of a semi-projective module inherits the property, while a direct sum of semi-projective modules need not be semi-projective. 
We show that a module $M=\oplus_{i \in I} M_{i}$ with $\operatorname{Hom}_{R}\left(M_{i}, M_{j}\right)=0$ for all $i \neq j$ in $I$ is semi-projective if and only if $M_{i}$ is semi-projective for all $i \in I$.

In the second part of this section we study direct sums of semi-projective modules over right Ore domains. We show that if $R$ is a right Ore domain, then every $R$ submodule of the right quotient division ring $Q$ is semi-projective. We prove that if $R$ is a right Ore domain with the right quotient division ring $Q \neq R$ and $X$ is a free right $R$-module then the right $R$-module $Q \oplus X$ is semi-projective if and only if there does not exist an $R$-epimorphism from $X$ to $Q$. It is also proved that if $R$ is a right Ore domain with right quotient division $\operatorname{ring} Q$ then the right $R$-module $Q \oplus R$ is semi-projective.

Third subsection is related directly to the second subsection. In this part we give general examples which are deduced from the preceding part. We observe that if $R$ is a PID (Principal Ideal Domain) with field of fractions $Q$ and $X$ is a proper submodule of $Q$ such that $R \subseteq X$, then $M=X \oplus R$ is finitely generated iff it is projective iff it is semi-projective iff it is direct projective.

Then, we define the semi-projective cover of an $R$-module $M$ and give some characterizations of semiperfect, perfect rings using semi-projective covers. We prove that a ring $R$ is (semi)perfect if and only if every (finitely generated) right $R$-module has a semi-projective cover.

Finally, the rings over which submodules of semi-projective right $R$-modules are semi-projective are investigated. Semihereditary, hereditary and semisimple rings also characterized using semi-projective modules.

\subsection{Some Properties of Semi-Projective Modules}

Definition 2.1.1 Let $R$ be a ring and let $X$ and $M$ be right $R$-modules. Then we shall say that $X$ is $M$-sprojective provided for every endomorphism $\alpha$ of $M$ and homomorphism $\beta: X \rightarrow M$ with $\beta(X) \subseteq \alpha(M)$ there exists a homomorphism $\gamma: X \rightarrow M$ such that $\beta=\alpha \gamma$.

Recall that an $R$-module $M$ is called semi-projective provided for all endomorphisms $\alpha$ and $\beta$ of $M$ with $\beta(M) \subseteq \alpha(M)$ there exists an endomorphism $\gamma$ of $M$ such that $\beta=\alpha \gamma$. 
It is clear that a module $M$ is semi-projective if and only if $M$ is $M$-sprojective. Note the following elementary fact which should be compared with Definition 1.2.1. We give the proof for completeness.

Proposition 2.1.2 Given $R$-modules $X$ and $M, X$ is $M$-sprojective if and only if for every submodule $L$ of $M$ such that $M / L$ embeds in $M$ and every homomorphism $\beta: X \rightarrow M / L$ there exists a homomorphism $\gamma: X \rightarrow M$ such that $\beta=\pi \gamma$, where $\pi: M \rightarrow M / L$ is the canonical projection.

Proof. The necessity is clear. Conversely, suppose that $X$ and $M$ have the stated condition. Let $\alpha$ be an endomorphism of $M$ and let $\beta: X \rightarrow M$ be a homomorphism such that $\beta(X) \subseteq \alpha(M)$. Let $N=\alpha(M)$ and let $K$ denote the kernel of $\alpha$. Then $N \cong M / K$. For each $x \in N$ there exists $m \in M$ such that $x=\alpha(m)$. Define the isomorphism $\theta: N \rightarrow M / K$ by $\theta(x)=m+K$. Note that $\pi=\theta \alpha$. By hypothesis, there exists a homomorphism $\gamma: X \rightarrow M$ such that $\pi \gamma=\theta \beta$. This implies that $\beta=\theta^{-1} \pi \gamma=\alpha \gamma$. It follows that $X$ is $M$-sprojective.

Proposition 2.1.3 Given a module $M$, every direct sum of $M$-sprojective modules is also $M$-sprojective.

Proof. Adapt the proof of Theorem 1.2.2.

It is not clear if there is an analogue of Proposition 1.2.3 for $M$-sprojective modules.

Definition 2.1.4 An $R$-module $M$ is called direct projective if for every direct summand $K$ of $M$ every epimorphism from $M$ to $K$ splits (see [7, 4.21] or [54, p. 365]).

It is pointed out in $[7, \mathrm{p} .33]$ that $M$ is direct projective if every submodule $N$ of $M$ such that $M / N$ is isomorphic to a direct summand of $M$ is also a direct summand of $M$. In [45, p. 57], direct projective modules are defined to be modules which satisfy the condition $\left(D_{2}\right)$. Note the following elementary fact.

Lemma 2.1.5 A module $M$ is direct projective if and only if for all endomorphisms $\alpha$ and $\beta$ of $M$ with $\beta(M) \subseteq \alpha(M)$ and $\alpha(M)$ a direct summand of $M$ there exists an endomorphism $\gamma$ of $M$ such that $\beta=\alpha \gamma$. 
Proof. Suppose first that $M$ is direct projective. Let $\alpha$ and $\beta$ be endomorphisms of $M$ with $\beta(M) \subseteq \alpha(M)$ and $K=\alpha(M)$ is a direct summand of $M$. Because $M$ is direct projective, there exists a homomorphism $\delta: K \rightarrow M$ such that $\alpha \delta=1_{K}$. Now $\gamma=\delta \beta$ is an endomorphism of $M$ such that $\beta=\alpha \gamma$.

Conversely, suppose that $M$ has the stated condition. Let $L$ be a direct summand of $M$ and $\varphi: M \rightarrow L$ be an epimorphism. There exists a submodule $L^{\prime}$ of $M$ such that $M=L \oplus L^{\prime}$. Let $\theta: M \rightarrow L$ be the canonical projection. Clearly $\theta(M)=L=$ $\varphi(M)$. By hypothesis, there exists an endomorphism $\lambda$ of $M$ such that $\theta=\varphi \lambda$. Let $\iota: L \rightarrow M$ denote the inclusion mapping. For all $y \in L, y=\theta(y)=\varphi \lambda(y)=\varphi \lambda \iota(y)$. It follows that $\varphi(\lambda \iota)=1$ and hence $\varphi: M \rightarrow L$ splits. Thus $M$ is direct projective.

Lemma 2.1.5 shows that we have the following hierarchy:

$$
\text { projective } \Rightarrow \text { quasi-projective } \Rightarrow \text { semi-projective } \Rightarrow \text { direct projective. }
$$

Examples 2.1.6 1. Every semisimple module, being quasi-projective, is semiprojective.

2. Let $\mathbb{N}$ denote the set of natural numbers $1,2, \ldots, \mathbb{Z}$ the ring of integers and $\mathbb{Q}$ the rational field. It is clear that, for any prime $p$ in $\mathbb{Z}$, the Prüfer $p$-group $\mathbb{Z}\left(p^{\infty}\right)$ is not direct projective and hence not semi-projective.

Let $N \leq M$ and $S=\operatorname{End}(M)$. In [34], Keskin-Tütüncü and Tribak denote the right ideal $\operatorname{Hom}(M, N)$ of $S$ by the notation $D(N)=\{\varphi \in S \mid \operatorname{Im} \varphi \subseteq N\}$ of $S$. They call a module $M$ dual Baer if for every $N \leq M$, there exists an idempotent $\epsilon$ in $S$ such that $D(N)=\epsilon S$.

Let $0 \neq \alpha: M \longrightarrow M$ be an endomorphism of $M$. We define the right ideal

$$
D(\alpha)=\{\varphi \in S \mid \operatorname{Im} \varphi \subseteq \operatorname{Im} \alpha\} \text { of } S .
$$

Actually $D(\alpha)=\operatorname{Hom}(M, \operatorname{Im} \alpha)$ for any endomorphism $\alpha$ of $M$. Of course if we take $N=\operatorname{Im} \alpha$, then $D(\alpha)=D(N)$. And, if $N$ is a nonzero direct summand of $M$, then there exists a nonzero idempotent $\epsilon$ of $S$ such that $D(\epsilon)=D(N)$. Note that $D(\alpha)=S$ if and only if $\alpha$ is epic. 
Lemma 2.1.7 Let $\epsilon$ be any idempotent endomorphism of a module $M$ with endomorphism ring $S$. Then $\epsilon S=D(\epsilon)$.

Proof. Let $\beta \in D(\epsilon)$. This means that $\beta$ is an endomorphism of $M$ such that $\beta(M) \subseteq \epsilon(M)$. Then $\epsilon=\epsilon^{2}$ implies that $(1-\epsilon) \beta(M) \subseteq(1-\epsilon) \epsilon(M)=0$. Thus $(1-\epsilon) \beta=0$ and hence $\beta=\epsilon \beta \in \epsilon S$.

Let $\alpha$ be an endomorphism of a module $M$ with endomorphism ring $S$ such that $\alpha(M)$ is a direct summand of $M$. Then $\alpha(M)=\epsilon(M)$ for some idempotent endomorphism $\epsilon$ of $M$. Clearly, $D(\alpha)=D(\epsilon)$. Now we consider an endomorphism of $M$ whose kernel is a direct summand of $M$.

Lemma 2.1.8 Let $\alpha$ be an endomorphism of a module $M$ with endomorphism ring $S$ such that the kernel of $\alpha$ is a direct summand of $M$. Then $D(\alpha)=\alpha S$.

Proof. Let $K=\operatorname{Ker} \alpha$. Then there exists a submodule $L$ of $M$ such that $M=K \oplus L$. Note that $\alpha(M)=\alpha(K)+\alpha(L)=\alpha(L)$. Let $\lambda: L \rightarrow \alpha(M)$ be the homomorphism defined by $\lambda(x)=\alpha(x)$ for all $x \in L$. Note that $\lambda$ is an isomorphism with $\alpha \lambda^{-1}=$ $1_{\alpha(M)}$. If $\beta$ is any endomorphism of $M$ such that $\beta(M) \subseteq \alpha(M)$ then $\gamma=\lambda^{-1} \beta$ is an endomorphism of $M$ such that $\beta=\alpha \gamma$. It follows that $D(\alpha)=\alpha S$.

It is given in $[7,4.20]$ that $M$ is semi-projective if and only if for every cyclic right ideal $I \subseteq \operatorname{End}(M), I=\operatorname{Hom}(M, I M)$, or equivalently, for any $f \in \operatorname{End}(M)$, $f \operatorname{End}(M)=\operatorname{Hom}(M, f(M))$. Then, it is easy to see that;

Lemma 2.1.9 Let $M$ be a module. $M$ is semi-projective if and only if $\alpha S=D(\alpha)$ for every nonzero $\alpha \in S$.

Definition 2.1.10 ([42]) A module $M$ is called Rickart if the kernel of any endomorphism of $M$ is a direct summand of $M$.

Therefore if we combine by Lemma 2.1.8 and Lemma 2.1.9, we get the following corollary;

Corollary 2.1.11 Let $M$ be a Rickart module. Then $M$ is semi-projective. 
Note that any Rickart module satisfies the sufficient condition of Examples 1.2.23, $(2)$.

Corollary 2.1.12 Let $M$ be a module with endomorphism ring $S$ such that $S$ is a von Neumann regular ring. Then $M$ is semi-projective.

Proof. If the endomorphism ring of $M$ is von Neumann regular, then $M$ is a Rickart module by [49, Theorem 4]. Thus $M$ is semi-projective by Corollary 2.1.11.

Definition 2.1.13 ([7]) A module $M$ is called extending provided every submodule is essential in a direct summand of $M$.

For example, semisimple modules are extending, as are uniform modules and injective modules.

Corollary 2.1.14 Every nonsingular extending module is semi-projective.

Proof. Let $M$ be any nonsingular extending module. Let $\alpha$ be any endomorphism of $M$ and let $K=\operatorname{Ker} \alpha$. There exists a direct summand $L$ of $M$ such that $K$ is an essential submodule of $L$. Now $M / K \cong \alpha(M) \leq M$, which is nonsingular. Thus $L / K$ is nonsingular. Since $L / K$ is also singular, hence $K=L$. This means that $K$ is a direct summand of $M$. Therefore $M$ is a Rickart module. By Corollary 2.1.11, $M$ is semi-projective.

Note that the $\mathbb{Z}$-module $\mathbb{Q}$ is semi-projective by Corollary 2.1.14 but since it is torsion-free and not free it is not quasi-projective from Theorem 1.2.19.

Now we investigate the direct summand property of semi-projective modules. It is not difficult to check that every direct summand of a semi-projective (respectively, direct projective) module is semi-projective (respectively, direct projective), as we show next for completeness.

Lemma 2.1.15 Every direct summand of a semi-projective (respectively, direct projective) module is also semi-projective (respectively, direct projective). 
Proof. Let a semi-projective module $M=M_{1} \oplus M_{2}$ be a direct sum of submodules $M_{1}, M_{2}$. Let $\alpha$ and $\beta$ be endomorphisms of $M_{1}$ such that $\beta\left(M_{1}\right) \subseteq \alpha\left(M_{1}\right)$. Now define endomorphisms $\lambda$ and $\mu$ of $M$ as follows: $\lambda\left(m_{1}+m_{2}\right)=\alpha\left(m_{1}\right)$ and $\mu\left(m_{1}+m_{2}\right)=$ $\beta\left(m_{1}\right)$ for all $m_{1} \in M_{1}$ and $m_{2} \in M_{2}$. Clearly $\mu(M) \subseteq \lambda(M)$. By hypothesis, there exists an endomorphism $\nu$ of $M$ such that $\mu=\lambda \nu$. If $\iota: M_{1} \rightarrow M$ denotes the inclusion mapping and $\pi: M \rightarrow M_{1}$ the canonical projection then let $\gamma$ denote the endomorphism $\pi \nu \iota$ of $M_{1} \cdot \alpha \gamma\left(m_{1}\right)=\alpha \pi \nu\left(m_{1}\right)$ for all $m_{1} \in M_{1}$. Since $\nu\left(m_{1}\right) \in M$ there exists $a_{1} \in M_{1}$ and $a_{2} \in M_{2}$ such that $\nu\left(m_{1}\right)=a_{1}+a_{2}$. Then $\alpha \gamma\left(m_{1}\right)=$ $\alpha \pi\left(a_{1}+a_{2}\right)=\alpha\left(a_{1}\right)=\lambda\left(a_{1}+a_{2}\right)=\lambda \nu\left(m_{1}\right)=\mu\left(m_{1}\right)=\beta\left(m_{1}\right)$ for all $m_{1} \in M$. Therefore $\beta=\alpha \gamma$. It follows that $M_{1}$ is a semi-projective module. The case of a direct summand of a direct projective module can be proved similarly.

It is stated in [52, Remark 2.3] that the direct sum of any collection of semiprojective modules is also semi-projective. This is not true in general. Haghany and Vedadi [25, p. 490] prove that if $R$ is a commutative domain with field of fractions $F$, then the $R$-module $R \oplus F$ is semi-projective. We shall show that an arbitrary direct sum of semi-projective modules need not be semi-projective, nor even direct projective. Then we shall go on to investigate when the direct sum of semi-projective modules is semi-projective.

Lemma 2.1.16 Let $R$ be a ring and let $X$ and $Y$ be $R$-modules such that the $R$ module $X \oplus Y$ is direct projective. Then every epimorphism $\varphi: X \rightarrow Y$ splits.

Proof. Clear by Lemma 1.2.26.

Corollary 2.1.17 Given any semi-projective $R$-module $Y$ which is not projective, there exists a projective $R$-module $X$ such that the $R$-module $X \oplus Y$ is not direct projective (and hence not semi-projective).

Proof. Take a semi-projective $R$-module $Y$ which is not projective. There exists a free $R$-module $X$ and an epimorphism $\varphi: X \rightarrow Y$. Suppose that $X \oplus Y$ is direct projective. By Lemma 2.1.16, $\varphi$ is a splitting epimorphism. So $\operatorname{Ker} \varphi \leq_{d} X$. By the isomorphism $X / \operatorname{Ker} \varphi \cong Y, Y$ is a projective $R$-module which contradicts to our assumption. Therefore the module $X \oplus Y$ is not direct projective. 
Corollary 2.1.18 The $\mathbb{Z}$-module $\mathbb{Z} \oplus \mathbb{Q}$ is semi-projective but the $\mathbb{Z}$-module $\mathbb{Z}^{(\mathbb{N})} \oplus \mathbb{Q}$ is not direct projective (and hence not semi-projective).

Proof. The module $\mathbb{Z} \oplus \mathbb{Q}$ is semi-projective by [25, p. 490]. As there is a non-splitting epimorphism from $\mathbb{Z}^{(\mathbb{N})}$ to $\mathbb{Q}$, Lemma 2.1 .16 shows that the $\mathbb{Z}$-module $\mathbb{Z}^{(\mathbb{N})} \oplus \mathbb{Q}$ is not direct projective.

Let $R$ be a Dedekind domain, let $M$ be a torsion $R$-module and let $P$ be a nonzero prime ideal in $R$. The $P$-primary component of $M$ is the set of all elements of $M$ that are annihilated by some positive power of $P$. The $P$-primary component is a submodule of $M$. By I. Kaplansky's well known result in [27, p. 332] that every torsion $R$-module is uniquely a direct sum of its $P$-primary submodules (in virtually the same way as for principal ideal rings), each $P$-primary submodule is a direct sum of cyclic primary $R$-modules that are also torsion.

Now we show that every finitely generated direct projective $\mathbb{Z}$-module is quasiprojective. In fact, more is true. Let $R$ be a Dedekind domain and let $M$ be a nonzero torsion cyclic $R$-module. From the above paragraph $M$ is a direct sum of cyclic primary $R$-modules. Let $X$ be a nonzero primary cyclic $R$-module. Being cyclic, $X \cong R / A$ for some proper ideal $A$ of $R$ and being primary, $P^{n} \subseteq A$ for some nonzero prime $P$ and a positive integer $n$. Now every nonzero ideal of $R$ is invertible and $A$ is a product of maximal ideals. It follows that $A=P^{k}$ for some positive integer $k$ with $1 \leq k \leq n$.

Theorem 2.1.19 Let $R$ be any Dedekind domain. Then the following statements are equivalent for an $R$-module $M$ which is a direct sum of cyclic submodules.

(i) $M$ is quasi-projective.

(ii) $M$ is semi-projective.

(iii) $M$ is direct projective.

Proof. (i) $\Rightarrow$ (ii) $\Rightarrow$ (iii) Clear.

(iii) $\Rightarrow$ (i) Let $M=\oplus_{i \in I} M_{i}$ be a direct sum of cyclic submodules $M_{i}(i \in I)$ and suppose that $M$ is direct projective. Suppose that $M$ is not torsion. Then there exists a direct summand $M_{j}(j \in I)$ which is not torsion. Since $M_{j}$ is cyclic $M_{j} \cong R / A$ 
for some right ideal $A$ of $R$. On the other hand $R / A$ is torsion for all nonzero right ideal $A$ of $R$. As $M_{j}$ is not torsion, then $M_{j} \cong R$ for $j \in I$. Now we claim that $M$ is a free $R$-module. If $M$ is not free then there exists $k \in I$ such that $M_{k} \nsucceq R$. That means that there exists $k \in I$ such that $M_{k}$ is torsion cyclic and hence there exists a non-splitting epimorphism $\varphi: M_{j} \rightarrow M_{k}$. Suppose that $\varphi$ is a splitting epimorphism. Since Ker $\varphi$ is a direct summand of $M_{j}, M_{k}$ is a projective $R$-module. By Proposition 1.6.8 $M_{k}$ is a torsion-free module that gives us a contradiction. By Lemma 2.1.16, $M_{j} \oplus M_{k}$ is not direct projective and, by Lemma 2.1.15 neither is $M$. Thus $M$ is free, so it is quasi-projective.

Now suppose that $M$ is a torsion $R$-module. From the well-known fact by Kaplansky [27, p. 332], $M$ can be written as a direct sum of its $P$-primary components for all nonzero prime (maximal) ideals $P$ of $R$. Let $N$ denote a nonzero Pprimary components of $M$ for any prime (maximal) ideal $P$ in $R$. By the above remarks, $N=\oplus_{\lambda \in \Lambda} N_{\lambda}$ for some index set $\Lambda$ and nonzero cyclic $P$-primary submodules $N_{\lambda}(\lambda \in \Lambda)$. Again by the above remarks, for each $\lambda \in \Lambda$ there exists a positive integer $m_{\lambda}$ such that $N_{\lambda} \cong R / P^{m_{\lambda}}$. Our claim is that $m_{\mu}=m_{\nu}$ for all different $\mu, \nu$. If $m_{\mu}<m_{\nu}$ for some $\mu \neq \nu$ then there is a non-splitting epimorphism $R / P^{m_{\nu}} \rightarrow R / P^{m_{\mu}}$. By Lemmas 2.1.15 and 2.1.16, $N$ is not a direct projective module and hence neither is $M$. Thus $m_{\mu}=m_{\nu}$ for all $\mu \neq \nu$ in $\Lambda$. Therefore $N=\oplus_{\mu \in \Lambda} \frac{R}{P^{m_{\mu}}}$. As $\frac{R}{P^{m_{\mu}}}$ is a quasi-projective module from Corollary 1.2.17, then $N$ is quasi-projective from Proposition 1.2.3. It is proved that every P-primary component of $M$ is quasi-projective and hence so also is $M$ from Theorem 1.2.20. This proves the result.

Corollary 2.1.20 Every finitely generated direct projective $\mathbb{Z}$-module is quasi-projective.

Proof. By Theorem 2.1.19.

If $R$ is a commutative domain which is not a field and $U$ a simple $R$-module then the $R$-module $R \oplus U$ is not a semi-projective module. To prove this, suppose that $R \oplus U$ is a semi-projective module. So it is direct projective. Since $U$ is simple, there exists $0 \neq x \in U$ such that $U=x R \cong \frac{R}{\operatorname{ann}_{R}(x)}$. Let us define the epimorphism 
$\varphi: R \longrightarrow U$ with $\varphi(r)=x r$. As $R \oplus U$ is direct projective, $\varphi$ is a splitting epimorphism. Then $\operatorname{Ker} \varphi=\operatorname{ann}_{R}(x)$ is a direct summand of a commutative domain $R$. So $\operatorname{ann}_{R}(x)=0$ or $\operatorname{ann}_{R}(x)=R$. But both gives us a contradiction. Therefore $R \oplus U$ can’t be semi-projective.

Here note that $\operatorname{Hom}_{R}(U, R)=0$ but $\operatorname{Hom}_{R}(R, U) \neq 0$. Compare this fact with the following result.

Remark 2.1.21 Let a module $M=\oplus_{i \in I} M_{i}$ be a direct sum of submodules $M_{i}(i \in$ I) such that $\operatorname{Hom}_{R}\left(M_{i}, M_{j}\right)=0$ for all $i \neq j$ in $I$. Then $M$ is semi-projective if and only if $M_{i}$ is semi-projective for all $i \in I$.

Proof. The necessity follows by Lemma 2.1.15. Conversely, suppose that $M_{i}$ is semiprojective for all $i \in I$. For each $k \in I$, let $\iota_{k}: M_{k} \rightarrow M$ denote the inclusion mapping and let $\pi_{k}: M \rightarrow M_{k}$ denote the canonical projection. Let $\alpha$ be any endomorphism of $M$. For all $j \neq k$ in $I, \pi_{j} \alpha \iota_{k} \in \operatorname{Hom}_{R}\left(M_{k}, M_{j}\right)=0$. Thus $\alpha\left(M_{k}\right) \subseteq M_{k}$ for all $k \in I$. And this implies that $\alpha(M)=\oplus_{i \in I} \alpha\left(M_{i}\right)$. Now let $\beta$ be an endomorphism of $M$ such that $\beta(M) \subseteq \alpha(M)$. For each $k \in I, \beta\left(M_{k}\right) \subseteq \alpha\left(M_{k}\right)$ and hence there exists an endomorphism $\gamma_{k}$ of $M_{k}$ such that $\alpha \iota_{k} \gamma_{k}=\beta \iota_{k}$. Define $\gamma=\sum_{k \in I} \iota_{k} \gamma_{k} \pi_{k}$ which is an endomorphism of $M$. It is easy to check that $\beta=\alpha \gamma$. It follows that $M$ is semi-projective.

Note the following corollary of Remark 2.1.21 which provides many examples of semi-projective modules as follows.

Corollary 2.1.22 Let $R$ be a prime right Goldie ring such that $R$ is not right primitive and let a right $R$-module $M=X \oplus Y$ be a direct sum of a torsion-free divisible submodule $X$ and a torsion semisimple submodule $Y$. Then $M$ is semiprojective.

Proof. Suppose that $R$ is prime right Goldie ring which is not primitive. So $R$ has a semisimple classical right quotient ring, by Theorem 1.5.10 (Goldie's Theorem). Say it $Q$. Since $X$ is a torsion-free module over prime right Goldie ring, then $X$ is nonsingular from Proposition 1.6.6 and $X$ is injective from Theorem 1.6.5. Therefore 
$X$ is semi-projective by Corollary 2.1.14. On the other hand, let $\varphi \in \operatorname{Hom}_{R}(Y, X)$ and let $y \in Y$. As $Y$ is torsion, there exists a regular element $d \in R$ such that $y d=0$ and hence $\varphi(y) d=\varphi(y d)=0$. Because $X$ is torsion-free, it follows that $\varphi(y)=0$ for all $y \in Y$ and hence $\varphi=0$. Thus $\operatorname{Hom}_{R}(Y, X)=0$. Now suppose that $\operatorname{Hom}_{R}(X, Y) \neq 0$. Then, since $X$ is a direct sum of copies of $Q$ from Proposition 1.6.7 and since $Y$ is semisimple, $\operatorname{Hom}_{R}(Q, V) \neq 0$ for some simple $R$-module $V$. Let $\alpha: Q \rightarrow V$ be a nonzero epimorphism. Because $R$ is not right primitive, $V$ has nonzero annihilator in $R$ and hence $V c=0$ for some regular element $c$ of $R$ from Theorem 1.5.9. Since $Q$ is divisible $R$-module over semiprime right Goldie ring $R$, $Q=Q c$ for the regular element $c \in R$. Then $\alpha(Q)=\alpha(Q c)=\alpha(Q) c=V c=0$ and hence $\alpha=0$, a contradiction. It follows that $\operatorname{Hom}_{R}(X, Y)=0$. By Remark 2.1.21, $M$ is semi-projective.

In particular, if $R$ is a prime ring and $R$ satisfies a polynomial identity ( $R$ is called a "PI ring" for short, (see [44])) then we have the following result.

Corollary 2.1.23 Let $R$ be a prime PI ring which is not Artinian and let a right $R$-module $M=X \oplus Y$ be a direct sum of a torsion-free divisible submodule $X$ and a torsion semisimple submodule $Y$. Then $M$ is semi-projective.

Proof. By Proposition 1.5.5 $R$ is right Goldie. Since $R$ is not Artinian, it is not a central simple algebra. So by Theorem 1.5.7 $R$ is not right primitive. Then apply Corollary 2.1.22.

We close this subsection by semi-Hopfian property of semi-projective modules.

Definition 2.1.24 ([7]) A module $M$ is called semi-Hopfian if the kernel of every epimorphism $\varphi: M \rightarrow M$ is a direct summand of $M$, equivalently, if $M / A \cong M$ with $A \leq M$, then $A$ is a direct summand of $M$.

Note the following fact.

Lemma 2.1.25 Every direct projective module is semi-Hopfian. 
Proof. It is clear since every epimorphism from $M$ to $M$ splits.

But the converse of this lemma is not true. For example, $\mathbb{Z} / n \mathbb{Z} \oplus \mathbb{Z}$ is a semiHopfian module but it is not direct projective.

Semi-Hopfian modules are semi-projective in the case of divisible modules over prime PI rings and this may be true more generally.

Proposition 2.1.26 Let $R$ be a prime PI ring. Then the following statements are equivalent for a divisible $R$-module $X$.

(i) $X$ is semi-projective.

(ii) $X$ is direct projective.

(iii) $X$ is semi-Hopfian.

(iv) $X$ is nonsingular.

Moreover, in this case $X$ is injective.

Proof. (i) $\Rightarrow$ (ii) By Lemma 2.1.5.

(ii) $\Rightarrow$ (iii) By Lemma 2.1.25.

(iii) $\Rightarrow$ (iv) Suppose that $X$ is not nonsingular. By Proposition 1.6.6, $X$ is not torsion-free. Then there exist a nonzero element $x \in X$ and a regular element $a \in R$ such that $x a=0$. From Proposition 1.5.4, $a R \leq_{e} R$. Since $R$ is right bounded by Proposition 1.5.5, there exists a nonzero right ideal $A$ of $a R$. Then from Proposition 1.5.6, there exists a nonzero element $c \in C \cap A$ where $C$ is the center of $R$. So $c$ is a nonzero central element with $c=a r$ for some $r \in R$ such that $x c=x a r=0$. Let $Y=\{u \in X: u c=0\}$. It is easy to check that $Y$ is a submodule of $X$. Now $X=X c$ because $c$ is a regular element of the prime $\operatorname{ring} R$ and $X$ is divisible. Define a mapping $\theta: X \rightarrow X$ by $\theta(w)=w c$ for all $w \in X$. It is easy to check that $\theta$ is an epimorphism with kernel $Y$. Suppose that $Y$ is a direct summand of $\mathrm{X}$. There exists a submodule $T$ of $X$ such that $X=Y \oplus T$. If we intersect both sides of the equality $X=X c$ by $Y$, then we get $Y=X c \cap Y$. Take $y \in Y=X c \cap Y$. There exists $x \in X$ such that $y=x c$. Since $X=Y \oplus T, x$ can be written as $x=y^{\prime}+t$, where $y^{\prime} \in Y$ and $t \in T$. Then $x c=y^{\prime} c+t c$. Since $y^{\prime} \in Y, x c=t c=y \in Y \cap T=0$. Therefore $Y=0$, a contradiction. Thus $Y$ is not a direct summand of $X$ and hence $X$ is not semi-Hopfian. 
(iv) $\Rightarrow$ (i) Suppose that $X$ is nonsingular. Then it is torsion-free by Proposition 1.6.6 and $X$ is injective by Proposition 1.6.5. Then $X$ is semi-projective by Corollary 2.1.14.

The last part follows from Proposition 1.6.5.

\subsection{Direct Sums of Semi-Projective Modules Over Ore Domains}

Let $X$ be a multiplicative set in a ring $R$. Then $X$ satisfies the right Ore condition provided that for each $x \in X$ and $r \in R$, there exist $y \in X$ and $s \in R$ such that $r y=x s$. A multiplicative set satisfying the right Ore condition is called a right Ore set. Any multiplicative set in a commutative ring is an Ore set. A regular element in a ring $R$ is any nonzero-divisor, i.e., any element $x \in R$ such that $\operatorname{ann}_{R}^{r}(x)=0$ and $\operatorname{ann}_{R}^{l}(x)=0$. If $X \subseteq R$ a multiplicative set of regular elements in $R$, then a right ring of fractions for $R$ with respect to $X$ is any overring $S \supseteq R$ such that every element of $X$ is invertible in $S$ and every element of $S$ can be expressed in the form $a x^{-1}$ for some $a \in R$ and $x \in X$. A right ring of fractions need not exist. If a multiplicative set of regular elements $X$ in a $\operatorname{ring} R$ is a right Ore set, then there exists a right ring of fractions for $R$ with respect to $X$. Converse is also true. A right ring of fractions $Q$ for $R$ with respect to $X$ is called classical right quotient ring (or $R$ is right order in $Q$ ) if $X$ is the set of all regular elements in $R$. So $R$ has a classical right quotient ring if and only if the set of regular elements in $R$ is a right Ore set. Every commutative ring has a classical quotient ring. In the case of commutative domain, the classical quotient ring is its quotient field. A non-commutative domain need not have a classical quotient ring, but if one exists, it will be a division ring. A right Ore domain is any domain $R$ in which the nonzero elements form a right Ore set. For any ring $R, R$ is a right Ore domain if and only if $R$ has a classical quotient ring which is a division ring. It is called right quotient division ring of $R$., (see [20]).

Given a submodule $X$ of the right $R$-module $Q$ we define

$$
\mathcal{O}(X)=\{q \in Q: q X \subseteq X\}
$$

Note that $\mathcal{O}(X)$ is a subring of $Q$. 
Lemma 2.2.1 Let a ring $R$ be a right order in a quotient ring $Q$ and let $X$ be a submodule of the right $R$-module $Q$ such that $X$ contains a regular element of $R$. Then $\alpha$ is an endomorphism of the right $R$-module $X$ if and only if there exists $q \in \mathcal{O}(X)$ such that $\alpha(x)=q x$ for all $x \in X$.

Proof. Given $q \in \mathcal{O}(X)$, it is clear that the mapping $\alpha: X \rightarrow X$ defined by $\alpha(x)=$ $q x(x \in X)$ is an $R$-homomorphism. For the converse, let $\beta$ be an endomorphism of $X$. By our assumption, let $c$ be a regular element of $R$ such that $c \in X$. There exists $p \in X$ such that $\beta(c)=p$. Let $x \in X \leq Q$. Then $x=a b^{-1}$ for some $a \in R$ and regular element $b \in R$. Note that $x b=a \in R$. Since $R$ is a right order in a quotient ring $Q$ (that means that $Q$ is a classical right quotient ring of $\mathrm{R}$ ), the set of regular elements in $R$ is a right Ore set by Theorem 1.3.7. For $a \in R$ and a regular element $c \in R$ there exist $a_{1} \in R$ and a regular element $c_{1} \in R$ such that $a c_{1}=c a_{1}$. Then $x b c_{1}=c a_{1}$ and hence

$$
\beta(x) b c_{1}=\beta\left(x b c_{1}\right)=\beta\left(c a_{1}\right)=\beta(c) a_{1}=p a_{1} .
$$

It follows that $\beta(x)=p a_{1} c_{1}^{-1} b^{-1}=p c^{-1} a b^{-1}=\left(p c^{-1}\right) x$. Thus $\beta(x)=\left(p c^{-1}\right) x$ for all $x \in X$. Note that $\left(p c^{-1}\right) X=\beta(X) \subseteq X$ and hence $p c^{-1} \in \mathcal{O}(X)$.

Proposition 2.2.2 Let $R$ be a right Ore domain with right quotient division ring $Q$. Then every submodule of the right $R$-module $Q$ is semi-projective.

Proof. Let $X$ be any submodule of $Q_{R}$. If $X=0$ then $X$ is clearly semi-projective. Suppose that $X \neq 0$. Let $0 \neq x \in X \leq Q$. For $a \in R$ and a regular element $b \in R, x=a b^{-1}$. Then $0 \neq x b=a \in X \cap R$ and this nonzero element $a=x b$ is a regular element because $R$ is a domain. So $X$ contains a regular element of $R$. Let $S=\operatorname{End}\left(X_{R}\right)$ and let $\alpha, \beta \in S$ with $\beta(X) \subseteq \alpha(X)$. If $\alpha=0$ then $\beta=0$ and hence $\beta \in \alpha S$, so $X$ is semi-projective. Suppose that $\alpha \neq 0$. By Lemma 2.2.1, there exist $p, q \in \mathcal{O}(X)$ with $\alpha(x)=p x$ and $\beta(x)=q x$ for all $x \in X$. Clearly $p \neq 0$ and

$$
q X=\beta(X) \subseteq \alpha(X)=p X \subseteq Q
$$

Because $p$ is nonzero we have $p^{-1} q \in Q$. Moreover, $p^{-1} q \in \mathcal{O}(X)$. Now define a mapping $\gamma: X \rightarrow X$ by $\gamma(x)=\left(p^{-1} q\right) x,(x \in X)$. Then $\gamma \in S$ by Lemma 2.2.1 and

$$
\alpha \gamma(x)=\alpha\left(p^{-1} q x\right)=p p^{-1} q x=q x=\beta(x), \quad \forall x \in X .
$$


Therefore $\beta=\alpha \gamma \in \alpha S$. It follows that $X$ is semi-projective.

The next lemma is elementary but is included for completeness.

Lemma 2.2.3 Let a module $M=X \oplus Y$ be the direct sum of a projective submodule $X$ and a submodule $Y$. Then $M$ is semi-projective if and only if for all endomorphisms $\alpha, \beta$ of $M$ with $\beta(X)=0$ and $\beta(Y) \subseteq \alpha(M)$ there exists an endomorphism $\gamma$ of $M$ such that $\beta=\alpha \gamma$.

Proof. The necessity is clear. Conversely, suppose that $M, X$ and $Y$ have the stated property. Let $\varphi, \theta$ be endomorphisms of $M$ with $\varphi(M) \subseteq \theta(M)$. Let $\iota: X \rightarrow M$ denote the inclusion mapping. Because $X$ is projective, there exists a homomorphism $\lambda: X \rightarrow M$ such that $\varphi \iota=\theta \lambda$. Let $\mu$ be the endomorphism $\lambda \pi$ of $M$, where $\pi: M \rightarrow X$ is the canonical projection. Then $\nu=\varphi-\theta \mu$ is also an endomorphism of $M$. Now

$$
\begin{gathered}
\nu(X)=(\varphi-\theta \mu)(X) \subseteq \varphi(X)-\theta \mu(X) \subseteq \varphi(X)-\theta \lambda \pi(X)=0 \text { and } \\
\nu(M)=\nu(Y)=(\varphi-\theta \mu)(Y) \subseteq \varphi(Y)-\theta \mu(Y) \subseteq \varphi(Y)-\theta \lambda \pi(Y)=\varphi(Y) \subseteq \varphi(M) \subseteq \theta(M) .
\end{gathered}
$$

By hypothesis, there exists an endomorphism $\gamma$ of $M$ such that $\nu=\theta \gamma$ and hence $\varphi=\theta(\mu+\gamma)$. Thus $M$ is semi-projective.

Before proving the next result we note the following well known fact which we shall prove for completeness.

Lemma 2.2.4 Let $R$ be a right Ore domain with right quotient division ring $Q \neq R$. Then $\operatorname{Hom}_{R}(Q, R)=0$.

Proof. Let $\varphi \in \operatorname{Hom}_{R}(Q, R)$. Since $Q$ is a divisible module $Q=Q c$ for each nonzero element $c$ of $R$ (Note that each nonzero element of $R$ is a regular element because $R$ is an Ore domain). Hence $\varphi(Q)=\varphi(Q c)=\varphi(Q) c \subseteq R c$ for each regular element $c \in R$. Suppose that $\varphi(Q) \neq 0$. We claim that $R \varphi(Q)$ is a minimal left ideal of $R$. Let $0 \neq J \leq R \varphi(Q)$ be the another left ideal of $R$. There exists an element $0 \neq c^{\prime} \in J \leq R$ which is regular. $R c^{\prime} \subseteq J \subseteq R \varphi(Q)=R \varphi\left(Q c^{\prime}\right)=R \varphi(Q) c^{\prime} \subseteq R c^{\prime}$. So $J=R c^{\prime}=R \varphi(Q)$. So $R \varphi(Q)$ is a minimal left ideal of $R$. Take an element 
$0 \neq d \in \varphi(Q)$ which is a regular element of $R .0 \neq R d \subseteq R \varphi(Q)$. Since $R \varphi(Q)$ is minimal, $R \varphi(Q)=R \varphi(Q) d=R d$ implies $R \varphi(Q)=R$. Then $R=R \varphi(Q)=$ $R \varphi(Q) c=R c$, so $R c=R$ and $R=Q$, a contradiction. Thus $\operatorname{Hom}_{R}(Q, R)=0$.

Let $R$ be a ring and $M$ an right $R$-module. We shall denote by $g\left(M_{R}\right)$ the least cardinal $\kappa$ such that there exists an index set $\Lambda$ of cardinality $\kappa$ and elements $m_{\lambda}(\lambda \in \Lambda)$ with $M=\sum_{\lambda \in \Lambda} m_{\lambda} R$. We have already noted that the $\mathbb{Z}$-module $\mathbb{Q} \oplus \mathbb{Z}^{(\mathbb{N})}$ is not semi-projective by Corollary 2.1.18. Compare this fact with the following result.

Theorem 2.2.5 Let $R$ be a right Ore domain with right quotient division ring $Q$ and let $X$ be a projective right $R$-module such that $g\left(X_{R}\right)<g\left(Q_{R}\right)$. Then the right $R$-module $M=Q \oplus X$ is semi-projective.

Proof. As $X$ is projective, it is a direct summand of a free $R$-module $Y$ such that $g\left(X_{R}\right) \leq g\left(Y_{R}\right)$. By Lemma 2.1.15 we can suppose without loss of generality that $X$ is free. Let $\left\{e_{i}\right\}_{(i \in I)}$ be a basis of $X$ with $|I|=\kappa$. First let us introduce some homomorphisms that we will need. If $\varphi$ is an endomorphism of $M$ then $\pi_{Q} \varphi \iota$ is an endomorphism of the $R$-module $Q$, where $\iota: Q \rightarrow Q \oplus X$ is the inclusion mapping and $\pi_{Q}: Q \oplus X \rightarrow Q$ the canonical projection. By Lemma 2.2.1 there exists $p \in \mathcal{O}(Q) \leq$ $Q$ such that $\pi_{Q} \varphi \iota(u)=p u$ for all $u \in Q$. Next note that if $\pi_{X}: Q \oplus X \rightarrow X$ is the canonical projection then $\pi_{X} \varphi \iota: Q \rightarrow X$ is an $R$-homomorphism. By using Lemma 2.2.4, $\pi_{X} \varphi \iota \in \operatorname{Hom}(Q, X)=\operatorname{Hom}(Q, \oplus R) \subseteq \sum \operatorname{Hom}(Q, R)=0$. This gives that $\pi_{X} \varphi \iota=0$. Then we get $\pi_{Q} \varphi(u, 0)=p u$ and $\pi_{X} \varphi(u, 0)=0$. Thus $\varphi(u, 0)=(p u, 0)$ for all $u \in Q$. And $\varphi(0, x)=\varphi\left(0, \sum_{I} e_{i} r_{i}\right)=\sum \varphi\left(0, e_{i}\right) r_{i}$.

Let $\alpha$ and $\beta$ be nonzero endomorphisms of $M$ such that $\beta(Q) \subseteq \alpha(M)$ and $\beta(X)=0$. There exist elements $q \in \mathcal{O}(Q) \subseteq Q$ and $q_{i} \in Q(i \in I)$ and elements $a_{i} \in X(i \in I)$ such that $\alpha(u, 0)=(q u, 0)(u \in Q)$ and $\alpha\left(0, e_{i}\right)=\left(q_{i}, a_{i}\right)$ for all $i \in I$ by above paragraph. Next again by previous paragraph there exists an element $q^{\prime} \in \mathcal{O}(Q) \leq Q$ such that $\beta(u, 0)=\left(q^{\prime} u, 0\right)$ for all $u \in Q$ and $\beta\left(0, e_{i}\right)=0$. Note that $\beta \neq 0$ implies that $q^{\prime} \neq 0$. Since $\beta(Q) \subseteq \alpha(M)$, for each element $u \in Q$, there exist an element $w \in Q$, a finite non-empty subset $F$ of $I$ and elements $r_{i} \in R(i \in F)$ 
such that

$\left(q^{\prime} u, 0\right)=\beta(u, 0)=\alpha\left(w, \sum_{i \in F} e_{i} r_{i}\right)=\alpha(w, 0)+\sum_{i \in F} \alpha\left(0, e_{i}\right) r_{i}=\left(q w+\sum_{i \in F} q_{i} r_{i}, \sum_{i \in F} a_{i} r_{i}\right)$

It follows that $q^{\prime} u=q w+\sum_{i \in F} q_{i} r_{i}$. Suppose that $q=0$. Then $q^{\prime} u=\sum_{i \in F} q_{i} r_{i}$. This implies that

$$
Q=q^{\prime} Q \subseteq \sum_{i \in F} q_{i} R
$$

In this case, $g\left(Q_{R}\right) \leq g\left(X_{R}\right)=|I|=\kappa$, a contradiction by our assumption.

Thus $q \neq 0$. There exist an element $w^{\prime} \in Q$, a finite non-empty subset $G$ of $I$ and elements $s_{i} \in R(i \in G)$ such that

$$
q^{\prime}=q w^{\prime}+\sum_{i \in G} q_{i} s_{i}=q\left(w^{\prime}+\sum_{i \in G} q^{-1} q_{i} s_{i}\right)=q \bar{q}
$$

where $\bar{q}=w^{\prime}+\sum_{i \in G} q^{-1} q_{i} s_{i} \in Q$. Now define a mapping $\gamma: M \rightarrow M$ by $\gamma(u, z)=$ $(\bar{q} u, 0)$ for all $u \in Q$ and $z \in X$. It is clear that $\gamma$ is an endomorphism of $M$. Moreover, for all $u \in Q, z \in X$ we have:

$$
\beta(u, z)=\beta(u, 0)=\left(q^{\prime} u, 0\right)=(q \bar{q} u, 0)=\alpha \gamma(u, z) .
$$

Thus $\beta=\alpha \gamma$. By Lemma 2.2.3, the module $M$ is semi-projective.

Theorem 2.2.5 has a number of immediate useful corollaries.

Corollary 2.2.6 Let $R$ be a right Ore domain with right quotient division ring $Q \neq R$ and let $X$ be a free right $R$-module. Then the right $R$-module $M=Q \oplus X$ is semi-projective if and only if there does not exist an epimorphism from $X$ to $Q$.

Proof. Suppose first that $M$ is not semi-projective. By Theorem 2.2.5, $g(Q) \leq g(X)$ and hence there is an epimorphism from $X$ to $Q$. Conversely, suppose that there is an epimorphism $\varphi: X \rightarrow Q$. Now suppose that $M$ is semi-projective. By Lemma 2.1.16, $\varphi$ splits. Then $Q_{R}$ is projective. So $\operatorname{Hom}_{R}(Q, R) \neq 0$, contradicting Lemma 2.2.4. Thus $M$ is not semi-projective.

Corollary 2.2.7 Let $R$ be a right Ore domain with right quotient division ring $Q$. Then the $R$-module $Q \oplus R$ is semi-projective. 
Proof. Suppose that $g\left(Q_{R}\right) \leq g\left(R_{R}\right)$. Clearly $g\left(R_{R}\right)=1$ and hence $g\left(Q_{R}\right)=1$. This means that $Q=q R$ for some $q \in Q$. In this case $Q \cong R$ as right $R$-modules and thus $Q \oplus R$ is a projective, and hence semi-projective $R$-module. If $g\left(R_{R}\right)<g\left(Q_{R}\right)$ then $Q \oplus R$ is semi-projective by Theorem 2.2.5.

Corollary 2.2.8 Let $R$ be a right Ore domain with right quotient division ring $Q$ and let $X$ be a finitely generated projective right $R$-module. Suppose that $R$ is right noetherian or left Ore. Then the $R$-module $Q \oplus X$ is semi-projective.

Proof. If $Q$ is not finitely generated then $g\left(Q_{R}\right)=\infty$ and $g\left(X_{R}\right)<g\left(Q_{R}\right)$. Then $Q \oplus X$ is semi-projective by Theorem 2.2.5. Suppose that $Q_{R}$ is finitely generated. If $R$ is right noetherian then finitely generated module $Q_{R}$ is also noetherian from Proposition 1.1.13. For any nonzero element $c \in R$, the ascending chain:

$$
R \subseteq c^{-1} R \subseteq c^{-2} R \subseteq \ldots
$$

must terminate. There exists a positive integer $n$ such that $c^{-n} R=c^{-n-1} R$. This gives $c^{-n-1}=c^{-n} b$ and hence $c b=1$ for some $b \in R$. Take any element $q \in Q$. Then $q=r x^{-1}$ for $r \in R$ and a regular element $x \in R$. As in the above sentence, there exists $b^{\prime} \in R$ such that $x b^{\prime}=1$. Hence $q=q x b^{\prime}=r b^{\prime} \in R$. So $Q=R$ and hence $Q \oplus X$ is a projective $R$-module. Now suppose that $R$ is a left Ore domain. In this case there exists a positive integer $k$ such that $Q=\left(c_{1}^{-1} r_{1}\right) R+\cdots+\left(c_{k}^{-1} r_{k}\right) R$ for some $r_{i} \in R, 0 \neq c_{i} \in R$ (they are regular), $(1 \leq i \leq k)$. By a standard argument we can suppose without loss of generality that $c_{1}=\cdots=c_{k}$. Then $Q=c_{1} Q=r_{1} R+\cdots+r_{k} R \subseteq R \subseteq Q$. Thus $Q=R$ and again $Q \oplus X$ is a projective $R$-module. In any case, $Q \oplus X$ is semi-projective.

\subsection{Some Examples}

We saw in Proposition 2.2.2 that if $R$ is a right Ore domain with right quotient division $\operatorname{ring} Q$ then every $R$-submodule $X$ of $Q$ is semi-projective. Moreover, Corollary 2.2.7 shows that if $X=Q$ then the $R$-module $X \oplus R$ is semi-projective. Of course, if $X=R$ then the $R$-module $X \oplus R$ is projective and hence semi-projective. We shall show in this section that in case $R=\mathbb{Z}$ then these $(\mathbb{Q}$ and $\mathbb{Z})$ are the only possible choices for a submodule $X$ of $\mathbb{Q}$ so that the $R$-module $X \oplus R$ is semi-projective. 
Let $R$ be any ring and consider an $R$-module $M=X \oplus R$ where $X$ is an $R$ module such that $\operatorname{Hom}_{R}(X, R)=0$. Let $\varphi$ be any endomorphism of the $R$-module $M$. Let $\iota_{X}: X \rightarrow M$ denote the inclusion mapping and let $\pi_{X}: M \rightarrow X$ and $\pi_{R}: M \rightarrow R$ denote the canonical projections. Note that $\pi_{R} \varphi \iota_{X} \in \operatorname{Hom}_{R}(X, R)=0$ and that $f=\pi_{X} \varphi \iota_{X} \in \operatorname{End}\left(X_{R}\right)$. Thus $\varphi(x, 0)=(f(x), 0)$ for all $x \in X$. Next there exist $y \in X$ and $a \in R$ such that $\varphi(0,1)=(y, a)$. It follows that

$$
\varphi(x, r)=(f(x)+y r, a r)(x \in X, r \in R),
$$

for that kind of modules $M$.

It is now easy to prove the following result.

Lemma 2.3.1 With the above notation, $\varphi$ is an endomorphism of $M$ if and only if there exists an endomorphism $f$ of $X$ and elements $y \in X, a \in R$ such that $\varphi(x, r)=(f(x)+y r$, ar $)$ for all $x \in X$ and $r \in R$.

Corollary 2.3.2 Let $R$ be a right Ore domain with right quotient division ring $Q$ and let $X$ be a nonzero submodule of the right $R$-module $Q$ such that $\operatorname{Hom}_{R}(X, R)=$ 0 . Let $M=X \oplus R$. Then $\varphi$ is an endomorphism of the $R$-module $M$ if and only if there exist $q \in \mathcal{O}(X), y \in X$ and $a \in R$ such that $\varphi(x, r)=(q x+y r$, ar $)$ for all $x \in X, r \in R$.

Proof. By Lemmas 2.2.1 and 2.3.1.

Now we prove a theorem about modules over a commutative PID (see also Theorem 2.1.19).

Theorem 2.3.3 Let $R$ be a PID with field of fractions $Q$ and let $X$ be a proper submodule of $Q$ such that $R \subseteq X$. Then the following statements are equivalent for the $R$-module $M=X \oplus R$.

(i) $M$ is finitely generated.

(ii) $M$ is projective.

(iii) $M$ is semi-projective.

(iv) $M$ is direct projective. 
Proof. (i) $\Rightarrow$ (ii) $\Rightarrow$ (iii) $\Rightarrow$ (iv) Clear by Lemma 2.1.5 and Proposition 1.6.8.

(iv) $\Rightarrow$ (i) Suppose that $X$ is not finitely generated. Since $Q_{R}$ is a uniform module, the proper submodule $X_{R}$ of $Q_{R}$ is also uniform. Suppose that $X$ is projective. As $R$ is a PID, $X$ is free. That means that $X=\bigoplus R_{R}$. Since uniform modules are indecomposable, $X$ is indecomposable. This is a contradiction. So $X$ being uniform implies that $X$ is not projective. Note that $\operatorname{Hom}_{R}(X, R)=0$. For, if $\varphi: X \rightarrow R$ is a nonzero homomorphism then $\varphi(X)$ is a nonzero projective ideal of $R$. This implies that $X / \operatorname{Ker} \varphi$ is also projective so $\operatorname{Ker} \varphi \leq_{d} X$. Suppose that $\operatorname{Ker} \varphi \neq 0$. As $X$ is uniform, $\operatorname{Ker} \varphi \leq_{e} X$. Then $X=\operatorname{Ker} \varphi$ and $\varphi(X)=0$, a contradiction. So $\operatorname{Ker} \varphi=0$ and hence $X \cong \varphi(X) \leq R$. Since $R$ is a PID, $X$ is finitely generated, that contradicts with the assumption. This contradiction shows that $\operatorname{Hom}_{R}(X, R)=0$. Let $T=\operatorname{End}\left(X_{R}\right)$. Suppose that $T=Q$. Then for any $0 \neq a \in R, X=X a$. It follows that the $R$-module $X$ is divisible. Since $R$ is PID, $X$ is injective so $X$ is a direct summand of $Q$. As $Q$ is indecomposable $X=Q$, a contradiction. Thus $T \neq Q$. There exists a prime element $p$ of $R$ such that $p$ is not a unit in $T$.

Now suppose that $X / R=p(X / R)$. Then $X=p X+R$. Let $\alpha$ denote the endomorphism of $M$ defined by $\alpha(x, r)=(p x+r, 0)$ for all $x \in X, r \in R$. Clearly $\alpha(M)=X \oplus 0=\pi(M)$ where $\pi: M \rightarrow X \oplus 0$ is the canonical projection. Suppose that $M$ is direct projective. There exist an endomorphism $\gamma$ of $M$ such that $\pi=\alpha \gamma$. By Lemma 2.3.1, there exists $q \in T=\operatorname{End}(X)$ and elements $y \in X$ and $a \in R$ such that $\gamma(x, r)=(q x+y r, a r)$ for all $x \in X$ and $r \in R$. Then;

$$
(x, 0)=\pi(x, r)=\alpha \gamma(x, r)=\alpha(q x+y r, a r)=(p q x+p y r+a r, 0) .
$$

So $1=p q$ for some $q \in T$, a contradiction since $p$ is not unit in $T$. Thus in this case $M$ is not direct projective.

Next suppose that $X / R \neq p(X / R)$. Since $Q / R$ is a torsion module over PID, it is isomorphic to the direct sum of injective envelopes of the simple modules $R / R q$, where $R q$ is a maximal ideal of $R$. The submodule $X / R$ of $Q / R$ is torsion and hence is a direct sum of its primary components. If $Y$ is the submodule of $X$ containing $R$ such that $Y / R$ is the $p$-primary component of $X / R$ then $Y=R\left(1 / p^{n}\right)+R=R\left(1 / p^{n}\right)$, for some positive integer $n$. If $Y^{\prime}$ is the submodule of $X$ containing $R$ such that $Y^{\prime} / R$ is the sum of the other primary components of $X / R$ then $X=Y+Y^{\prime}$. Moreover 
$Y^{\prime} / R=p\left(Y^{\prime} / R\right)$ so that $Y^{\prime}=p Y^{\prime}+R$. Let $\beta$ be the endomorphism of $M$ defined by $\beta(x, r)=\left(p x+r / p^{n}, 0\right)$ for all $x \in X, r \in R$. So $\beta(M)=\beta(X \oplus R) \subseteq X \oplus 0$. For all $y^{\prime} \in Y^{\prime}$ there exist $z \in Y^{\prime}, b \in R$ such that $y^{\prime}=p z+b$ and hence $\left(y^{\prime}, 0\right)=$ $(p z+b, 0)=\left(p z+\frac{p^{n} b}{p^{n}}, 0\right)=\beta\left(z, p^{n} b\right)$. Next note that $\left(1 / p^{n}, 0\right)=\left(p 0+\frac{1}{p^{n}}, 0\right)=\beta(0,1)$. Take $\left(y+y^{\prime}, 0\right) \in X \oplus 0$ such that $y \in Y$ and $y^{\prime} \in Y^{\prime}$. There exist $z \in Y^{\prime}, b, r \in R$ such that $y=\frac{r}{p^{n}}$ and $y^{\prime}=p z+b$.

$\left(y+y^{\prime}, 0\right)=(y, 0)+\left(y^{\prime}, 0\right)=\left(\frac{r}{p^{n}}, 0\right)+(p z+b, 0)=\beta(0, r)+\beta\left(z, p^{n} b\right)=\beta\left(z, p^{n} b+r\right)$ which is in $\beta(X \oplus R)=\beta(M)$. It follows that $\beta(M)=X \oplus 0=\pi(M)$ for the canonical projection $\pi$. Suppose that $M$ is direct projective. Then there exists an endomorphism $\delta$ of $M$ such that $\pi=\beta \delta$. Lemma 2.3.1 gives that $1=p q^{\prime}$ for some $q^{\prime} \in T$, a contradiction. Thus $M$ is not direct projective in this case also. We conclude that $M$ is not direct projective if $M$, and hence $X$, is not finitely generated.

Corollary 2.3.4 Let $R$ be a PID with field of fractions $Q$ and let $X$ be any nonzero submodule of $Q$. Then the following statements are equivalent for the $R$-module $M=X \oplus R$.

(i) $M$ is semi-projective.

(ii) $M$ is direct projective.

(iii) $X \cong R$ or $X \cong Q$.

Proof. (i) $\Rightarrow$ (ii) By Lemma 2.1.5.

(ii) $\Rightarrow$ (iii) Since $X \neq 0$, there exists $0 \neq x \in X \subseteq Q$. Then $0 \neq x=c z^{-1}$ for some regular element $z$ in $R$ and an element $c \in R$. So $0 \neq c=x z$ in $X$. Clearly;

$$
M \cong M c^{-1}=X c^{-1} \oplus R c^{-1} \cong X c^{-1} \oplus R .
$$

In addition, $R=(R c) c^{-1} \subseteq X c^{-1}$. Suppose that $X c^{-1}$ is a proper submodule of $Q$. Then by applying Theorem 2.3.3 for $X c^{-1}, X c^{-1}$ is finitely generated module. Since finitely generated modules over PID are isomorphic to direct sum of cyclic modules and since $X$ is indecomposable then $X c^{-1} \cong R$. If $X c^{-1}=Q$ then $X c^{-1} \cong Q$ and it follows that $X \cong R$ or $X \cong Q$.

(iii) $\Rightarrow$ (i) By Corollary 2.2.7. 
Up to now, we concerned with rings $R$ and $R$-modules $M$ such that $M=X \oplus R$ for some $R$-module $X$ with the property that $\operatorname{Hom}_{R}(X, R)=0$. It has been seen that such modules $M$ need not be semi-projective. In other words, from Lemma 2.1 .9 , if $S$ is the endomorphism ring of the $R$-module $M$ then in many cases there exists $\alpha \in S$ such that $\alpha S \neq D(\alpha)$. Now we show that $\alpha S$ is an essential submodule of the right $S$-module $D(\alpha)$.

Theorem 2.3.5 Let $R$ be a ring, $X$ an $R$-module, $M$ the $R$-module $X \oplus R$ and let $S$ be the endomorphism ring of the $R$-module $M$. Then $\alpha S$ is an essential submodule of the $S$-module $D(\alpha)$ for every $0 \neq \alpha \in S$.

Proof. There exists an epimorphism $\varphi: F=R^{(\Lambda)} \longrightarrow M$. Let $0 \neq \alpha \in S$ and $0 \neq g \in D(\alpha)$. By the projectivity of $F$, there exists a homomorphism $h: F \longrightarrow M$ such that $\alpha h=g \varphi$. Moreover if $g \varphi \epsilon_{\lambda}=0$ for all index set $\lambda \in \Lambda$ where $\epsilon_{\lambda}$ is the inclusion map from $R$ to $F$, then $g \varphi(R)=0$. Then

$$
g(M)=g \varphi(F)=g \varphi(\oplus R)=\oplus g \varphi(R)=0
$$

a contradiction. So there exists an index $\lambda \in \Lambda$ such that $g \varphi \epsilon_{\lambda} \neq 0$. Consider the projection map $\pi: M \longrightarrow R$. Hence $\alpha\left(h \epsilon_{\lambda} \pi\right)=g\left(\varphi \epsilon_{\lambda} \pi\right)$ is a nonzero element of $\alpha S \cap g S$, which shows that $\alpha S$ is essential in $D(\alpha)$.

\subsection{Semi-Projective Covers}

Recall that a module $M$ has a projective cover $P$, if there is an epimorphism $f: P \longrightarrow M$ such that $P$ is projective and $\operatorname{Ker} f$ is small in $P$. A ring $R$ is called right perfect if every right $R$-module has a projective cover. Perfect rings were characterized by H. Bass in [3]. In 1967, L. E. T. Wu and J. P. Jans introduced the quasi-projective cover as follows in [57]: The module $P$ is called a quasi-projective cover of a module $M$ if, there exists an epimorphism $f: P \longrightarrow M$ such that (1) $P$ is quasi-projective (2) $\operatorname{Ker} f$ is small in $P(3)$ if $0 \neq B \subseteq \operatorname{Ker} f$, then $P / B$ is not quasi-projective. Note that similar to the case of projective covers, quasi-projective covers of a module need not exist. For example, the $\mathbb{Z}$-module $M=\oplus_{k} \mathbb{Z} / p^{k} \mathbb{Z}$ does not have a quasi-projective cover (see [11, Example 4]). Also, it is not known whether quasi-projective cover of a module (if it exists) is unique up to isomorphism. L. 
E. T. Wu and J. P. Jans proved in [57, Proposition 2.6] that when the projective cover $f: P \longrightarrow M$ exists, then the quasi-projective cover of $M$ exists and is unique. This quasi-projective cover is given by the induced map $f^{\prime}: P / T \longrightarrow M$, where $T$ is the largest fully invariant submodule of $P$ contained in $\operatorname{Ker} f$.

In 1970; K. R. Fuller and D. A. Hill [14, Theorem 4.1], J. Golan [16, Theorem 3.1] and A. Koehler [35, Corollary 1.2] proved that (the condition (3) is not needed for the proof) a ring $R$ is right perfect if every right $R$-module has a quasi-projective cover and they also investigated semiperfect rings via quasi-projective covers of finitely generated modules. After that, in 1983, T. G. Faticoni studied quasi-projective covers in [11] and in 1996, W. Xue defined the locally projective cover (without the condition (3)) and proved that a ring $R$ is right perfect if and only if every right $R$-module has a locally projective cover in [59, Theorem 3.10]; he also investigated semiperfect rings via locally projective covers.

In this chapter firstly we define semi-projective covers and investigate right perfect rings. We say that a module $P$ is a semi-projective cover of any module $M$ if, there exists an epimorphism $f: P \longrightarrow M$ such that $P$ is semi-projective and Ker $f$ is small in $P$. Since this definition does not stipulate an analogue of the condition (3), the semi-projective cover may not be unique up to isomorphism. Clearly, every (quasi-)projective cover is a semi-projective cover. But the converse is not true. Since the $\mathbb{Z}$-module $\mathbb{Q}$ is semi-projective (see, Corollary 2.1 .14 ), $\mathbb{Q}_{\mathbb{Z}}$ is a semi-projective cover of itself and of the $\mathbb{Z}$-module $\mathbb{Q} / \mathbb{Z}$.

In this part of the dissertation, we give some characterizations of semiperfect and perfect rings by using semi-projective covers. We obtain that a ring $R$ is right perfect if and only if every right $R$-module has a semi-projective cover and $R$ is semiperfect if and only if every finitely generated right (left) $R$-module has a semi-projective cover. This characterizations have been completely inspired by the earlier related studies from [3], [11], [14], [16], [17], [35], [57] and [59].

The following theorem is an analogue of [17, Theorem 2.2].

Theorem 2.4.1 Let $M$ be a module and let $f: P \longrightarrow M$ be an epimorphism with $P$ projective. Then

1. $M$ is projective if and only if $P \oplus M$ is semi-projective. 
2. $M$ has a projective cover if and only if $P \oplus M$ has a semi-projective cover.

Proof. (1) Assume $M$ is projective. Then clearly $P \oplus M$ is semi-projective. Conversely assume that $P \oplus M$ is semi-projective, so it is direct projective. Then the epimorphism $f: P \longrightarrow M$ splits from Lemma 2.1.16. Then $\operatorname{Ker} f \leq_{d} P$ from Definition 1.2.4. Thus by the isomorphisms $M \cong P / \operatorname{Ker} f \cong T \leq_{d} P$ and by Theorem 1.2.2, $M$ is projective.

(2) For the necessity, assume that the epimorphism $\mu: P^{\prime} \longrightarrow M \longrightarrow 0$ be the projective cover of $M$, with $P^{\prime}$ projective and $\operatorname{Ker} \mu \ll P^{\prime}$. Then the epimorphism $i d_{P} \oplus \mu: P \oplus P^{\prime} \longrightarrow P \oplus M$ be the semi-projective cover of $P \oplus M$. For the sufficiency we will use the Koehler's technique in [35, Theorem 1.1]. By hypothesis, there exists an epimorphism $g: Q \longrightarrow P \oplus M$ such that $Q$ is semi-projective and $\operatorname{Ker} g$ is small in $Q$. Let $\pi$ be the projection map from $P \oplus M$ to $P$. Because of the projectivity of $P$, there exists a monomorphism $\alpha: P \longrightarrow Q$ such that $\pi g \alpha=1_{P}$. That means that $\pi g$ splits. By Proposition 1.2.5, $Q \cong P \oplus \operatorname{Ker}(\pi g)$. Without loss of generality, we can assume $Q=P \oplus \operatorname{Ker}(\pi g)$. Let $\bar{M}=\operatorname{Ker}(\pi g)$ and $g_{1}=\left.g\right|_{\bar{M}}$.

$$
g_{1}(\bar{M})=\left.g\right|_{\bar{M}}(\bar{M})=g(\bar{M})=g(\operatorname{Ker}(\pi g))=\operatorname{Ker} \pi=M
$$

implies that $g_{1}: \bar{M} \longrightarrow M$ is an epimorphism. Now we will prove that $\bar{M}$ is the projective cover of $M$ with the epimorphism $g_{1}$. Since $\operatorname{Ker} g=\operatorname{Ker} g_{1}, \operatorname{Ker} g_{1}$ is small in $\bar{M}$. Since $P$ is projective, there is a homomorphism $f^{\prime}: P \longrightarrow \bar{M}$ such that $g_{1} f^{\prime}=f$, namely the following diagram is commutative:

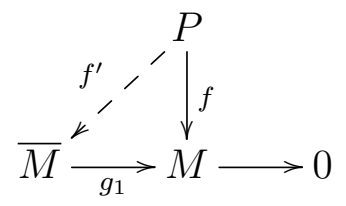

Since $f$ is epic, $g_{1} f^{\prime}(P)=f(P)=M$, so $f^{\prime}(P)+\operatorname{Ker} g_{1}=\bar{M}$. Since $\operatorname{Ker} g_{1}$ is small in $\bar{M}, f^{\prime}(P)=\bar{M}$. Therefore since $Q$ is semi-projective and $f^{\prime}: P \longrightarrow \bar{M}$ is an epimorphism then $\bar{M}$ is projective by (1).

Corollary 2.4.2 If every (finitely generated) module has a semi-projective cover, then every (finitely generated) module has a projective cover. 
Proof. Suppose that every (finitely generated) module has a semi-projective cover. Take a (finitely generated) module $M$. Since every (finitely generated) module is an epimorphic image of a (finitely generated) free module, there exists an epimorphism $\varphi: F \longrightarrow M \longrightarrow 0$. From the hypothesis, $F \oplus M$ has a semi-projective cover. Then by Theorem 2.4.1 (2), $M$ has a projective cover.

In [16], J. S. Golan characterized perfect (semiperfect) rings as rings over which every (finitely generated) module has a quasi-projective cover. Now, we characterize perfect and semiperfect rings by using semi-projective cover.

Corollary 2.4.3 1. A ring $R$ is semiperfect if and only if every finitely generated right (left) $R$-module has a semi-projective cover.

2. A ring $R$ is right perfect if and only if every right $R$-module has a semiprojective cover.

Proof. By Corollary 2.4.2.

Now applying the same technique in the proof of [17, Theorem 3.1], we get the following, where $R_{n}$ is the ring of $n \times n$ matrices over $R$ :

Theorem 2.4.4 If $R$ is a ring, then the following conditions are equivalent for $R$ :

1. $R$ is semiperfect.

2. For all $n \geq 1$, every cyclic right (left) $R_{n}$-module has a semi-projective cover.

3. There exists an $n>1$ such that every cyclic right (left) $R_{n}$-module has a semi-projective cover.

Proof. $(1) \Rightarrow(2)$ : Assume that $R$ is semiperfect. By Theorem 1.2.13, $R_{n}$ semiperfect for all $n \geq 1$. Then every cyclic right (left) $R_{n}$-module has a semi-projective cover for all $n \geq 1$ by Corollary 2.4.3.

$(2) \Rightarrow(3)$ : Trivial

$(3) \Rightarrow(1)$ : Let $n>1$ and assume that every cyclic right $R_{n}$-module has a semiprojective cover. Let $N$ be a cyclic right $R$-module. There exists a right ideal $L$ of $R$ such that $N \cong R / L$. Now we will show that $R / L$ has a semi-projective cover. $L_{n}$ is 
the right ideal of $R_{n}$ consisting of all matrices with entries from $L$. Let $e_{i j} \in R_{n}$ be the matrix with $1_{R}$ in the $(i, j)$ position and zeros elsewhere. Then $R_{n} / e_{11} L_{n} \cong P \oplus M$, where $M \cong e_{11} R_{n} / e_{11} L_{n}$ and $P \cong \sum_{i=2}^{n} e_{i i} R_{n} . P$ is clearly a projective $R_{n}$-module and the map $\lambda: P \longrightarrow M$ which sends $\left[a_{i j}\right]$ to $e_{12}\left[a_{i j}\right]+e_{11} L_{n}$ is an $R_{n}$-epimorphism. By hypothesis $P \oplus M$ has a semi-projective cover and by Theorem 2.4.1 (2), $M$ has a projective cover over $R_{n}$. Call the epimorphism $g: P^{\prime} \longrightarrow M$ where $P^{\prime}$ is a projective $R_{n}$-module and Kerg is small in $P^{\prime}$. And $g\left(P^{\prime} e_{11}\right)=g\left(P^{\prime}\right) e_{11}=M e_{11}$ which is isomorphic to $R / L$ as an $R$-module. Let $\alpha$ be the isomorphism from $M e_{11}$ to $R / L . P^{\prime}$ is a projective $R_{n}$-module, so $P^{\prime} e_{11}$ is a projective $R$-module by [28] and $\alpha g$ is an epimorphism with $\operatorname{Ker}(\alpha g) \ll P^{\prime} e_{11}$. Therefore, $(\alpha g): P^{\prime} e_{11} \longrightarrow R / L$ is a projective cover of $R / L$, proving (1).

In 2014 Yousif, Amin and Ibrahim studied D3-cover of a module in [60].

\subsection{Rings Over Which Submodules of Semi-projective Right R-modules are Semi-projective}

Golan in [17, Theorem 4.4] proved that a ring $R$ is right hereditary if and only if every submodule of a projective right $R$-module is quasi-projective if and only if every principal right ideal of $\operatorname{End}(F)$ is quasi-projective for any free right $R$-module $F$ and in [17, Theorem 4.3] he also proved that $R$ is right semihereditary if and only if every finitely generated submodule of a projective right $R$-module is quasiprojective if and only if every principal right ideal of $R_{n}$ is quasi-projective, for all $n \geq 1$. Now we will adapt these two results to semi-projective modules.

First we need the following result of R. R. Colby and E. A. Rutter Jr. from [8, Theorem 2.3];

Theorem 2.5.1 [8] A ring $R$ is right (semi)hereditary if and only if the endomorphism ring of every (finitely generated) free right $R$-module is a right PP-ring.

Theorem 2.5.2 The following conditions are equivalent for a ring $R$ :

1. $R$ is right hereditary.

2. Every submodule of a projective right $R$-module is semi-projective. 
3. Every principal right ideal of End $(F)$ is semi-projective for any free right $R$ module $F$.

Proof. $(1) \Rightarrow(2)$ : Obvious from the definition of right hereditary rings.

$(2) \Rightarrow(1)$ : Assume that every submodule of a projective right $R$-module is semiprojective and let $N$ be a submodule of a projective right $R$-module $P$. There exist a projective module $F$ which maps epimorphically onto $N . F \oplus N$ is a submodule of a projective module $F \oplus P$ and so is semi-projective from the hypothesis. From Theorem 2.4.1(1), $N$ is projective. Therefore $R$ is right hereditary.

$(1) \Rightarrow(3)$ : It is clear from Theorem 2.5.1.

$(3) \Rightarrow(1)$ : Assume that every principal right ideal of $\operatorname{End}(F)=E$ is semi-projective for any free right $R$-module $F$. Let $F$ be any free right $R$-module. $F \oplus F$ is free with the endomorphism ring which is isomorphic to $E_{2}$ ( 2 by 2 matrix with entries belong to $E$ ). From the hypothesis, every principal right ideal of $E_{2}$ is semi-projective. We will show that every principal right ideal of $E$ is projective. Let $a \in E$ and $K$ be principal right ideal of $E_{2}$ generated by $\left[\begin{array}{ll}a & 0 \\ 0 & 1\end{array}\right]$. Then $K$ is semi-projective over $E_{2}$ and so $K e_{11} \cong a E \oplus E$ is semi-projective over $E$. Since $E$ maps epimorphically onto $a E$, this implies that $a E$ is projective by Theorem 2.4 .1 (1). Then every principal right ideal of $E=\operatorname{End}(F)$ is projective ( $E$ is a PP-ring) for any free module $F$. By Theorem 2.5.1, $R$ is right hereditary.

Theorem 2.5.3 The following conditions are equivalent for a ring $R$ :

1. $R$ is right semihereditary.

2. Every finitely generated submodule of a (finitely generated) projective right $R$ module is semi-projective.

3. Every finitely generated (principal) right ideal of $R_{n}$ is semi-projective for all $n \geq 1$.

Proof. $(1) \Rightarrow(3)$ : As $R$ is right semihereditary, $R_{n}$ is also right semihereditary for all $n \geq 1$, from Theorem 1.2.13.

The other parts of the proof is along the same lines as that of Theorem 2.5.2. 
Submodules of semi-projective modules need not be semi-projective as the following example shows.

Example 2.5.4 Let $M$ be the semi-projective $\mathbb{Z}$-module $\mathbb{Z} / p^{3} \mathbb{Z} \oplus \mathbb{Z} / p^{3} \mathbb{Z}$, where $p$ is any prime integer. (Since $p^{3} \mathbb{Z}$ is a two sided ideal of $\mathbb{Z}$, then $Z / p^{3} \mathbb{Z}$ is quasiprojective $\mathbb{Z}$-module. By Proposition $1.2 .15, \mathbb{Z} / p^{3} \mathbb{Z} \oplus \mathbb{Z} / p^{3} \mathbb{Z}$ is quasi-projective then it is semi-projective.) Let $N$ be the submodule $p \mathbb{Z} / p^{3} \mathbb{Z} \oplus \mathbb{Z} / p^{3} \mathbb{Z}$ of $M$. Suppose that $N_{\mathbb{Z}}$ is semi-projective, so it is direct projective. Then the nonzero epimorphism $f$ : $\mathbb{Z} / p^{3} \mathbb{Z} \longrightarrow p \mathbb{Z} / p^{3} \mathbb{Z}$ defined by $f\left(x+p^{3} \mathbb{Z}\right)=p x+p^{3} \mathbb{Z}$ will split and so $0 \neq \operatorname{Ker} f$ will be a direct summand of the hollow module $\mathbb{Z} / p^{3} \mathbb{Z}$. Hence $\operatorname{Ker} f=0$, a contradiction. Therefore $N$ is not semi-projective.

In [17], it is studied at rings $R$ over which (finitely generated) submodules of a quasi-projective right $R$-module are quasi-projective. Similarly we investigate the rings over which every (finitely generated) submodule of a semi-projective right $R$ module is semi-projective. This condition is stronger than right hereditary and in fact we will show in Theorem 2.5.5 that every factor ring of that kind of ring is right (semi)hereditary. If $R$ is right perfect, then the converse also holds. We will now characterize these rings in detail by the same methods as in [17] and [18].

Theorem 2.5.5 Let $R$ be a ring. If every (finitely generated) submodule of a semiprojective right $R$-module is semi-projective, then every factor ring of $R$ is right (semi)hereditary.

Proof. Note that if $M$ and $N$ are two $R / I$-modules where $I$ is two sided ideal of $R$, then $\operatorname{Hom}_{R / I}(M, N)=\operatorname{Hom}_{R}(M, N)$. Now let $I$ be a two sided ideal of $R$ and $S=R / I$. Let $P$ be a projective right $S$-module with (finitely generated) $S$ submodule $M . P$ is semi-projective right $R$-module by Proposition 1.2.18. Then by hypothesis, $M$ is a semi-projective right $R$-module and so it is a semi-projective right $S$-module. Therefore by Theorem 2.5.2 (Theorem 2.5.3), $S$ is right (semi-)hereditary. 
Note that the arguments in the proofs below are based on the techniques of $[18$, Proposition 2.2, Theorem C, Corollary 2.4, Lemma 2.5, Corollary 2.6 and Theorem D], respectively.

Proposition 2.5.6 Let $R$ be a ring. If every submodule of a semi-projective right $R$-module is semi-projective and $H$ is a right $T$-nilpotent two-sided ideal of $R$, then $H^{2}=0$.

Proof. Since $H$ is a right $T$-nilpotent two-sided ideal of $R, H=R H$ is small in $R$ by Lemma 1.1.10. Therefore $H$ is contained in the Jacobian Radical $J(R)=J$ of $R$. Let $S=R / H^{2}$. By Theorem 2.5.5, $S$ is right hereditary, so in particular $J / H^{2}$ is a projective right $S$-module since it is a right ideal of $S$. Let $U_{S}=J / H^{3}$ and $V_{S}=H^{2} / H^{3}$. As $U / V$ is $S$ isomorphic to $J / H^{2}, U / V$ is projective over $S$. Therefore $V$ is a direct summand of $U$. On the other hand, $V \subseteq U H \ll U$ implies that $V \ll U$. Thus $V=0$. This proves that $H^{2}=H^{3}$. Again by applying Lemma 1.1 .10 for an $R$-module $H^{2}, H^{2}=H^{3}$ is small in $H^{2}$. And so $H^{2}=0$.

Theorem 2.5.7 If $R$ is right perfect and every submodule of a semi-projective right $R$-module is semi-projective, then every singular right $R$-module is injective.

Proof. Let $M$ be a singular right $R$-module. Let $H$ be an essential right ideal of $R, \lambda: H \longrightarrow R$ be the inclusion map and $\alpha: H \longrightarrow M$ any nonzero $R$ homomorphism. We want to find a homomorphism $\beta: R \longrightarrow M$ such that $\beta \lambda=\alpha$. Write $\operatorname{Ker} \alpha=K$ and assume that $K$ is not essential in $R$. Then there exists a nonzero right ideal $I$ of $R$ such that $K \cap I=0$. Since $H$ is essential in $R, I^{\prime}=H \cap I$ is nonzero and $I^{\prime} \cap K=H \cap I \cap K=0$, i.e $\left.\operatorname{Ker} \alpha\right|_{I^{\prime}}=0$. Thus the restriction of $\alpha$ to $I^{\prime}$ is a monomorphism and so $I^{\prime} \cong \alpha\left(I^{\prime}\right)$, which is a submodule of a singular module $M$ and so is singular. On the other hand, $R$ is ,in particular, right hereditary from Theorem 2.5.2 and so is nonsingular from Proposition 1.1.8. Therefore $I^{\prime} \leq$ $R$ is also nonsingular. Now $I^{\prime}=0$, a contradiction. Thus $K$ is essential in $R_{R}$. Since $R$ is a right perfect ring $R / J(R)$ is semisimple and $J(R)$ is right $T$-nilpotent. By Proposition 2.5.6 $J(R)^{2}=0$ and by Proposition 1.1.6 $\operatorname{Soc}\left(R_{R}\right)=r_{R}(J(R))$. Therefore $J(R) \subseteq \operatorname{Soc}\left(R_{R}\right)$. So $J(R) \subseteq K$. Therefore $R / K$ is a right $R / J(R)$-module 
and thus semisimple from Proposition 1.1.5. The map $\alpha$ induces a monomorphism $\bar{\alpha}: H / K \longrightarrow M$. Since $R / K$ is semisimple, $H / K$ is a direct summand of $R / K$ and so we have a canonical projection $\pi: R / K \longrightarrow H / K$. Finally let $\nu: R \longrightarrow R / K$ be the canonical epimorphism. Then $\bar{\alpha} \pi \nu: R \longrightarrow M$ and for every $h \in H, \bar{\alpha} \pi \nu(h)=$ $\bar{\alpha} \pi(h+K)=\bar{\alpha}(h+K)=\alpha(h)$. So $\beta=\bar{\alpha} \pi \nu$ is the homomorphism we seek.

Corollary 2.5.8 If $R$ is right perfect and every submodule of a semi-projective right $R$-modules is semi-projective, then $Z(M)$ is a direct summand of $M$ for every right $R$-module $M$.

Lemma 2.5.9 Let $R$ be a left perfect ring. Assume that every finitely generated submodule of a semi-projective right $R$-module is semi-projective. If $e$ and $f$ are idempotents of $R$ with $e R$ and $f R$ indecomposables, and $e R f$ and $f R e$ are nonzero, then $e R \cong f R$ and in fact this isomorphism is given by left multiplication by any nonzero element of eRf or $f R e$.

Proof. Let $a$ and $b$ be elements of $R$ such that eaf and fbe are nonzero. Define $\alpha: e R \longrightarrow f R$ by $e r \mapsto f b e r$. Then $e R \oplus f b e R$ is a finitely generated submodule of $R \oplus R$ and so is semi-projective by hypothesis. Then $\alpha$ splits since it is epic from $e R$ to $f b e R$ by Theorem 2.1.16. Therefore, since $e R$ is indecomposable, $\alpha$ is monic. Similarly the homomorphism $\beta: f R \longrightarrow e R$ given by $f r \mapsto e a f r$ is in fact a monomorphism. Now we will show that $e R=\operatorname{Im} \beta \alpha=$ eafbeR. Clearly we have the descending chain $e R \supseteq \beta \alpha(e R) \supseteq(\beta \alpha)^{2}(e R) \supseteq \ldots$ of principal right ideals of $R$ which must terminate since $R$ is left perfect (see Theorem 1.2.10). Then there exists an integer $n$ such that $(\beta \alpha)^{n}(e R)=(\beta \alpha)^{n+1}(e R)$. In particular for all $r \in R$ $(\beta \alpha)^{n}(e r)=(\beta \alpha)^{n+1}\left(e r^{\prime}\right)$ for some $r^{\prime} \in R$ and so $e r-\beta \alpha\left(e r^{\prime}\right) \in \operatorname{Ker}(\beta \alpha)^{n}$. But $\beta$ and $\alpha$ are monomorphisms. Thus $e r=(\beta \alpha)\left(e r^{\prime}\right)$ and so $e R=\beta \alpha(e R)=e$ afbe $R$. Therefore $\beta$ is an epimorphism. This completes the proof.

Corollary 2.5.10 Let $R$ be a left perfect ring. Assume that every finitely generated submodule of a semi-projective right $R$-module is semi-projective and $e$ is an idempotent of $R$ with e $R$ indecomposable. Then eRe is a division ring. 
Theorem 2.5.11 If $R$ is left perfect and every finitely generated submodule of a semi-projective right $R$-module is semi-projective, then $R_{\mathbb{Z}}=S \oplus J(R)$, where $S$ is a semisimple subring of $R$ containing 1 .

Proof. Since $R$ is left perfect, there exists a set $\left\{e_{1}, \ldots, e_{n}\right\}$ of orthogonal idempotents of $R$ such that $R=\oplus_{i=1}^{n} e_{i} R$, each $e_{i} R$ is indecomposable and $e_{i} R / \operatorname{Rad}\left(e_{i} R\right)$ is a simple right $R$-module by [2, Theorem 27.12, $(\mathrm{a} \Rightarrow \mathrm{b})]$. Furthermore we have the $\mathbb{Z}$-decomposition $R=\oplus_{i=1}^{n} \oplus_{j=1}^{n} e_{i} R e_{j}$. Fix some index $k, 1 \leq k \leq n$ and let $S_{k}=\sum e_{i} R e_{j}$, where the sum ranges over all indices $i, j$ such that $e_{i} R \cong e_{j} R \cong e_{k} R$. Then $S_{k}$ is a subring of $R$ which is isomorphic to the full matrix rings $\left(e_{k} R e_{k}\right)_{t}$, where $t$ is the number of different indices $i$ in the above sum. Since $e_{k} R e_{k}$ is a division ring by Corollary 2.5.10, $S_{k}$ is a simple ring. Now let $S=\sum_{k=1}^{n} S_{k}$. Then this is a subring of $R$ and, since each $S_{k}$ is simple, $S$ is semisimple and contains $1=e_{1}+\ldots+e_{n}$. Let $T=\sum e_{i} R e_{j}$, where the sum ranges over all indices $i, j$ such that $e_{i} R \nsucceq e_{j} R$. We will be done if we can show that $J(R)=T$. Let $e_{i} a e_{j} \notin J(R)$. Then $e_{i} a e_{j} R \nsubseteq e_{i} J(R)=\operatorname{Rad}\left(e_{i} R\right)$. Since $e_{i} R / \operatorname{Rad}\left(e_{i} R\right)$ is simple and $\operatorname{Rad}\left(e_{i} R\right)$ is maximal in $e_{i} R, e_{i} a e_{j} R=e_{i} R$. Now the homomorphism $e_{i} R \longrightarrow e_{j} R$ given by $e_{i} r \mapsto e_{i} a e_{j} r$ is an isomorphism since the kernel is both small and a direct summand. Thus by the definition of $T, e_{i} a e_{j} \notin T$. Hence $T \subseteq J(R)$. To show that $T=J(R)$ it suffices to show that $T$ is a two sided ideal of $R$. $T$ is clearly closed under addition. Let $e_{j} a e_{i} \in T$ and consider $0 \neq y=e_{j} a e_{i} b e_{k}$. If $e_{k} R \nRightarrow e_{j} R$, then $y \in e_{j} R e_{i} \subseteq T$. So $T R \subseteq T$. Hence assume that $e_{k} R \cong e_{j} R$. If $\beta: e_{k} R \longrightarrow e_{i} R$ and $\alpha: e_{i} R \longrightarrow e_{j} R$ are the $R$-homomorphisms respectively given by $e_{k} r \mapsto e_{i} b e_{k} r$ and $e_{i} r \mapsto e_{j} a e_{i} r$ then by the same reasoning in Lemma 2.5.9, each of these maps is a monomorphism. Let $e_{k} r \in e_{k} R$. Then $\alpha \beta\left(e_{k} r\right)=\alpha\left(e_{i} b e_{k} r\right)=e_{j} a e_{i} b e_{k} r=y e_{k} r$. Namely $\alpha \beta$ is just the map given by left multiplication by $y$ and so by Lemma 2.5.9 is an isomorphism from $e_{k} R$ to $e_{j} R$. Therefore $\alpha$ must be an epimorphism so is an isomorphism from $e_{i} R$ to $e_{j} R$, a contradiction since $e_{j} a e_{i} \in T$. Hence $y=0$ for $e_{k} R \cong e_{j} R$ and so $T R \subseteq T$. A similar proof shows that $R T \subseteq T$. So $T$ is two sided ideal.

If $M$ is an $R$-module and $A$ is any non-empty set, then the direct product (sum) of $|A|$-copies of $M$ is denoted by $M^{A}\left(M^{(A)}\right)$, and $M$ is said to be $\sum$-semi-projective if $M^{(A)}$ is semi-projective for any $A$. Now using the same proof as in Theorem 7 in 
[58], we can give the following result, which generalizes Theorem 7 in [58].

Theorem 2.5.12 If $R$ is a ring over which submodules of $\Sigma$-semi-projective modules are direct-projective, then every factor ring of $R$ is right hereditary.

Proof. Let $I$ be an ideal of $R$ and $S=R / I$. Let $P_{S}$ be a projective $S$-module with a submodule $N$. For any non-empty set $A, P^{(A)}$ is still a projective $S$-module. By Propositon 1.2.18, $P^{(A)}$ is quasi-projective and hence semi-projective $R$-module. Therefore $P$ is a $\sum$-semi-projective $R$-module. By hypothesis, $N$ is direct projective $S$-module. Then by Theorem 1.2.12, $S$ is right hereditary.

In [35] and [36] Koehler characterized semisimple rings using quasi-projective modules and quasi-injective modules. Using her results and ideas we have our concluding result by using the same techniques in [58].

Theorem 2.5.13 The following conditions are equivalent for a ring $R$ :

1. $R$ is semisimple.

2. Every (finitely generated) right $R$-module is semi-projective.

3. Every 2-generated right $R$-module is semi-projective.

4. The direct sum of two semi-projective right $R$-modules is semi-projective.

5. The direct sum of two quasi-projective right $R$-modules is semi-projective.

6. For all $n \geq 1$, every cyclic right $R_{n}$-module is semi-projective.

7. There exists some $n>1$ such that every cyclic right $R_{n}$-module is semiprojective.

Proof. $(1) \Rightarrow(2) \Rightarrow(3),(1) \Rightarrow(4) \Rightarrow(5)$ and $(1) \Rightarrow(6) \Rightarrow(7)$ are trivial.

$(3) \Rightarrow(1)$ : Let $I$ be a right ideal of $R$. Since $R \oplus R / I$ semi-projective by hypothesis, $R / I$ is projective by Theorem 2.4.1 (1). Hence $I$ is a direct summand of $R$, proving (1).

(5) $\Rightarrow(1)$ : If $T$ is a simple $R$-module, then $R \oplus T$ is semi-projective by (5). It follows from Theorem 2.4.1 (1) that $T$ is projective, hence $R$ is semisimple. 
$(7) \Rightarrow(1)$ : Let $I$ be a right ideal of $R$. We show that $R / I$ is projective. First we denote by $I_{n}$ the right ideal of $R_{n}$ consisting of all $n$ by $n$ matrices with entries from $I$. Let $e_{i j} \in R_{n}$ be the matrix unit. Then $R_{n} / e_{11} I_{n} \cong P \oplus M$ as right $R_{n}$-modules, where $M=e_{11} R_{n} / e_{11} I_{n}$ and $P=\sum_{i=2}^{n} e_{i i} R_{n}$ (as the proof of Theorem 2.4.4). Hence $P \oplus M$ is semi-projective $R_{n}$-module by (7). Clearly, $P$ is projective and there is an $R_{n}$-epimorphism $P \longrightarrow M$ via $\left(r_{i j}\right) \mapsto e_{21}\left(r_{i j}\right)+e_{11} I_{n}$. It follows from Theorem 2.4.1 that $M$ is projective $R_{n}$-module. In [28], S. M. Kaye proved that there is a Morita equivalence between $R_{n}$-modules and $R$-modules via $M \mapsto M e_{11}$. Since $M$ is a projective $R_{n}$-module, $M e_{11} \cong R / I$ is a projective $R$-module. 


\section{SGQ-PROJECTIVE MODULES}

Let $M$ be an $R$-module with the endomorphism $\operatorname{ring} S$ and let $0 \neq s \in S$. Recall the definition of the right ideal;

$$
D(s)=\{\varphi \in S \mid \operatorname{Im} \varphi \subseteq \operatorname{Im} s\}
$$

of $S . D(s)=\operatorname{Hom}(M, \operatorname{Im} s)$ for any endomorphism $s$ of $M$. If we take $N=\operatorname{Im} s$, then $D(s)=D(N)$. And, if $N$ is a nonzero direct summand of $M$, then there exists a nonzero idempotent $e$ of $S$ such that $D(e)=D(N)$. Note that $D(s)=S$ if and only if $s$ is epic; and $D(e)=e S$ for every idempotent element $e$ in $S$.

In this part of the dissertation we introduce $S G Q$-projective modules by means of $D(s)$ as an another generalization of quasi-projective modules. Let $M$ be any module. Then we call $M S G Q$ - projective if for any $0 \neq s \in S$, there exists a right ideal $X$ of $S$ such that $D(s)=s S \oplus X$.

\subsection{Some Properties of SGQ-projective Modules}

In this subsection we introduce some basic properties of SGQ-projective modules. And we characterize semisimple rings with the help of SGQ-projective modules. We also investigate the direct sum and direct summand properties of SGQ-projective modules.

Let $N \leq M$. We will say that $N$ is co-M-cyclic if there is a submodule $K$ of $M$ with $M / N \cong K$. Clearly, every submodule of the Prüfer $p$-group $\mathbb{Z}\left(p^{\infty}\right)$ is co- $M$-cyclic.

Let $M$ and $N$ be two modules. Let us say that $N$ is $M-\mathcal{K}$-projective if the following diagram

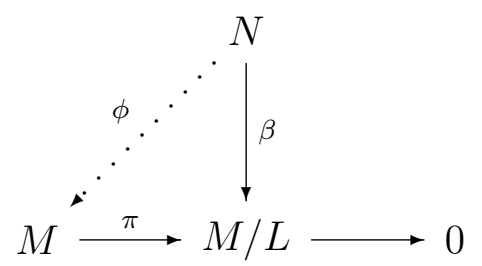

is commutative, where $L$ is a co- $M$-cyclic submodule of $M$ and $\pi$ is the natural epimorphism. It is easy to show that $N$ is $M-\mathcal{K}$-projective if and only if the following 
diagram

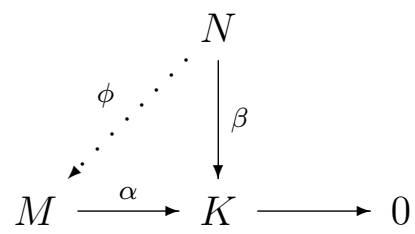

is commutative, where $K$ is any submodule of $M$. Note that in the above diagram actually $K$ is an $M$-cyclic submodule of $M$. We say that $M$ is quasi $\mathcal{K}$-projective (or semi-projective) if $N=M$ in the above definition. Note that saying " $N$ is $M-\mathcal{K}$ projective" is equivalent to saying that " $N$ is $M$-principally projective" in [52] and is equivalent to saying that " $N$ is $M$-semi-projective" in [53] and " $N$ is $M$-sprojective" in Definition 2.1.1.

Lemma 3.1.1 (see also [52, Lemma 2.2]) Let $M$ and $N$ be two modules with $S=$ $\operatorname{End}(M)$. Then $N$ is $M$-K- -projective if and only if for every nonzero $s \in S$, $\operatorname{sHom}(N, M)=\{f: N \longrightarrow M \mid \operatorname{Imf} \subseteq \operatorname{Ims}\}$.

Proof. Assume $N$ is $M$ - $\mathcal{K}$-projective. Let $0 \neq s \in S$. Clearly $\operatorname{sHom}(N, M) \subseteq\{f$ : $N \longrightarrow M \mid \operatorname{Im} f \subseteq \operatorname{Im} s\}$. Let $f: N \longrightarrow M$ and $\operatorname{Im} f \subseteq \operatorname{Im} s$. Since $N$ is $M-\mathcal{K}$ projective, there exists a homomorphism $t: N \longrightarrow M$ such that $s t=f$. Therefore $f \in \operatorname{sHom}(N, M)$.

Conversely, assume that $\operatorname{sHom}(N, M)=\{f: N \longrightarrow M \mid \operatorname{Im} f \subseteq \operatorname{Ims}\}$. Let $\varphi: N \longrightarrow K \leq M$ be a homomorphism and $s: M \longrightarrow K$ an epimorphism. By hypothesis, since $\operatorname{Im} \varphi \subseteq \operatorname{Im} s$ there exists a homomorphism $f: N \longrightarrow M$ such that $\varphi=s f$. Therefore $N$ is $M$ - $\mathcal{K}$-projective.

Definition 3.1.2 Let $M$ be any module. Then we call $M S G Q$-projective if for any $0 \neq s \in S$, there exists a right ideal $X$ of $S$ such that $D(s)=s S \oplus X$.

Comparing Lemma 2.1.9 with this definition, we get the following hierarchy;

Corollary 3.1.3 Every quasi-projective module is semi-projective and every semiprojective module is $S G Q$-projective.

$$
\text { quasi-projective } \Rightarrow \text { semi-projective } \Rightarrow \text { SGQ-projective. }
$$

Theorem 3.1.4 Let $M$ be a module with $S$ is regular. Then $M$ is $S G Q$-projective. 
Proof.It is clear from Corollaries 2.1.12 and 3.1.3.

Examples 3.1.5 1. The $\mathbb{Z}$-module $\mathbb{Q}_{\mathbb{Z}}$ is semi-projective and hence $S G Q$-projective, but it is not quasi-projective by Theorem 1.2.19.

2. Let $R$ be any integral domain with quotient field $F \neq R$. Then $M_{R}=F \oplus R$ is semi-projective by [25] and hence SGQ-projective.

3. Let $R$ be a prime ring such that its Martindale ring of quotients $Q$ satisfies $\operatorname{Hom}_{R}(Q, R) \neq 0$. Then $Q_{R}$ is semi-projective by [25] and hence $S G Q$ projective.

Now some explanations are given to use in the next part;

Note that if any nonzero homomorphism $s: M \longrightarrow M$ is right invertible, there exits an endomorphism $f \in S$ such that $s f=1_{M}$. So $s S=S$. Then $M=s f(M) \subseteq$ $s(M) \subseteq M$. Therefore $s$ is epic. For the converse, $M$ needs to be SGQ-projective:

Lemma 3.1.6 Let $M$ be an SGQ-projective module. Any nonzero homomorphism $s: M \longrightarrow M$ is epic if and only if $s$ is right invertible.

Proof. Assume that $0 \neq s: M \longrightarrow M$ is epic. Then $D(s)=S$ and $s(M)=M$. If $M$ is $S G Q$-projective, then $D(s)=s S \oplus X$ for some right ideal $X$ of $S$. Now there exists a nonzero idempotent $e \in S$ such that $s S=e S$. So $s=e t$, for some $t \in S$. Then $M=s(M)=e t(M) \subseteq e(M) \subseteq M$. Thus $e$ is epic and so $D(e)=S$. On the other hand since $e$ is an idempotent, $D(e)=e S=s S$. Therefore $S=s S$ and $s$ is right invertible. Converse is clear from the above paragraph.

Definition 3.1.7 ([7]) $M$ is called Hopfian if every surjective endomorphism of $M$ is an isomorphism.

It is clear that every Hopfian module is semi-Hopfian. Recall that direct-projective modules are also semi-Hopfian.

Lemma 3.1.8 If $M$ is SGQ-projective, then $M$ is semi-Hopfian. 
Proof. Take a nonzero epimorphism $s: M \longrightarrow M$. Since $M$ is SGQ-projective and $s$ is epic, $s$ is right invertible from Lemma 3.1.6. There exists a homomorphism $f \in S$ such that $s f=1$. Then $\operatorname{Im} f \oplus \operatorname{Ker} s=M$ so kernel of $s$ is a direct summand of $M$ for every epimorphism $s \in S$. Therefore $M$ is semi-Hopfian.

Note that the converse of this lemma cannot be true; namely, any semi-Hopfian module need not be $S G Q$-projective. The $\mathbb{Z}$-module $M_{\mathbb{Z}}=\mathbb{Z} / n \mathbb{Z} \oplus \mathbb{Z}$ with $n$ any positive integer is not $S G Q$-projective (see Example 3.1.13) but it is semi-Hopfian.

Proof. We will show that $M=\mathbb{Z} / n \mathbb{Z} \oplus \mathbb{Z}$ is a Hopfian module. Take an epimorphism $\varphi: M \rightarrow M \rightarrow 0$ with $\varphi(\overline{0}, 1)=(\bar{a}, b)$ and $\varphi(\overline{1}, 0)=(\bar{c}, 0)$, where $a, b, c \in \mathbb{Z}$. Then for all $x, y \in \mathbb{Z}$

$$
\varphi(\bar{x}, y)=x \varphi(\overline{1}, 0)+y \varphi(\overline{0}, 1)=x(\bar{c}, 0)+y(\bar{a}, b)=(\overline{x c+y a}, y b) .
$$

And $\operatorname{Ker} \varphi=\{(\bar{x}, y) \mid x c+y a \equiv 0(\bmod n), y b=0\}$. Since $\varphi$ is an epimorphism, then $b \neq 0$. Therefore $\operatorname{Ker} \varphi=\{(\bar{x}, 0) \mid x c \equiv 0(\bmod n)\}$. In the case of $\bar{c}=0$, $\operatorname{Ker} \varphi \subseteq \mathbb{Z} / n \mathbb{Z} \oplus 0 . M \cong M / \operatorname{Ker} \varphi \cong \mathbb{Z} / m \mathbb{Z} \oplus \mathbb{Z}$ implies that $m=n$. So $\operatorname{Ker} \varphi=0$. In the case of $\bar{c} \neq 0, x$ divides $n$. If $n=x, \operatorname{Ker} \varphi=0$. If $n \neq x$, then $n=x d$ for some $d \in \mathbb{Z}$. So $M \cong M / \operatorname{Ker} \varphi \cong \mathbb{Z} / d \mathbb{Z} \oplus \mathbb{Z}$, which is a contradiction. Therefore $M$ is Hopfian, so it is semi-Hopfian.

Every discrete module is quasi-discrete, but the converse is not always true. It is proved in [45, Lemma 5.1] that if $M$ is quasi-discrete, semi-Hopfian module then $M$ is discrete.

Proposition 3.1.9 Let $M$ be a quasi-discrete $S G Q$-projective module. Then $M$ is discrete.

Proof. Since $M$ is SGQ-projective, then $M$ is semi-Hopfian. Thus by [45, Lemma 5.1], $M$ is discrete.

Example 3.1.10 The Prüfer p-group $\mathbb{Z}\left(p^{\infty}\right)$ is not $S G Q$-projective by Proposition 3.1.9.

Any factor module of an SGQ-projective module need not be SGQ-projective: 
Example 3.1.11 Let $R$ be a discrete valuation ring. Let $K$ be its quotient field with $K_{R}$ quasi-projective. As an $R$-module, $K / R$ is quasi-discrete but not discrete by [45, Example 4.46]. So, $K / R$ cannot be $S G Q$-projective by Proposition 3.1.9. But $K_{R}$ is $S G Q$-projective.

It is natural to investigate whether or not a notion is inherited by direct summands and direct sums. We show that a direct summand of an $S G Q$-projective module inherits the property. It is shown that a direct sum of $S G Q$-projective modules is not $S G Q$-projective, in general. We focus on when a direct sum of $S G Q$-projective modules is also $S G Q$-projective.

Any direct summand of an $S G Q$-projective module is $S G Q$-projective:

Theorem 3.1.12 Let $M$ be any module and $K$ any direct summand of $M$. If $M$ is $S G Q$-projective, then $K$ is $S G Q$-projective.

Proof. Let $i: K \longrightarrow M$ be the inclusion map and $\pi: M \longrightarrow K$ be the projection map. Let $T=\operatorname{End}\left(K_{R}\right)$ and $S=\operatorname{End}\left(M_{R}\right)$. For all $\tau \in T$, let $\hat{\tau}=i \tau \pi$ and note that $\pi \hat{\tau}_{\mid K}=\tau$. Let $\tau \in T$. It is not hard to see that $D_{T}(\tau)=\left\{\alpha \in T \mid \hat{\alpha} \in D_{S}(\hat{\tau})\right\}$. Since $M$ is $S G Q$-projective, $D_{S}(\hat{\tau})=\hat{\tau} S \oplus A$ for some right ideal $A$ of $S$. Let $B=\left\{\pi \lambda_{\mid K} \mid \lambda \in A\right\}$. Assume $\alpha \in D_{T}(\tau)$. Then $\hat{\alpha}=\hat{\tau} \sigma+\lambda$ for some $\lambda \in A$ and $\sigma \in S$. Now $\alpha=\pi \hat{\alpha}_{\mid K}=\pi(\hat{\tau} \sigma)_{\mid K}+\pi \lambda_{\mid K}=\tau \pi \sigma_{\mid K}+\pi \lambda_{\mid K}=\tau\left(\pi \sigma_{\mid K}\right)+\pi \lambda_{\mid K} \in \tau T+B$. Therefore $D_{T}(\tau) \subseteq \tau T+B$. Clearly, $B \subseteq D_{T}(\tau)$. Therefore $D_{T}(\tau)=\tau T+B$. Now we will show that $\tau T \cap B=0$. Let $\tau \delta \in B$ with $\delta \in T$. Then $\tau \delta=\pi \lambda_{\mid K}$ for some $\lambda \in A$. Note that $\tau \delta \pi=\pi \lambda i \pi$ and $i \pi \sigma=\sigma$ for all $\sigma \in D_{S}(\hat{\tau})$. Therefore $\hat{\tau} \hat{\delta}=i \tau \pi i \delta \pi=i \tau \delta \pi=i \pi \lambda i \pi=\lambda i \pi \in A \cap \hat{\tau} S=0$. So, $\tau \delta=(\hat{\tau} \hat{\delta})_{\mid K}=0$. Thus $D_{T}(\tau)=\tau T \oplus B$. Hence $K$ is $S G Q$-projective.

Any direct sum of two $S G Q$-projective modules need not be $S G Q$-projective as we see in the following example.

Example 3.1.13 Let $n \mathbb{Z}$ be an ideal of $\mathbb{Z}$. Let $M_{\mathbb{Z}}=\mathbb{Z} / n \mathbb{Z} \oplus \mathbb{Z}$. It is not hard to see that $M$ is not semi-projective. By Theorem 2.3.5, $\alpha \operatorname{End}(M)$ is an essential submodule of the End $(M)$-module $D(\alpha)$ for every nonzero endomorphism $\alpha$ of $M$. Therefore $M$ cannot be $S G Q$-projective. Note that $\mathbb{Z} / n \mathbb{Z}$ and $\mathbb{Z}$ are $S G Q$-projective. 
In this vein we give the following:

Theorem 3.1.14 Let $A_{i}$ be $S G Q$-projective module for each $i \in I$ such that $M=$ $\oplus_{i \in I} A_{i}$. If every $A_{i}$ is fully invariant in $M$, then $M$ is $S G Q$-projective.

Proof. Let $S=\operatorname{End}(M)$ and $S_{i}=\operatorname{End}\left(A_{i}\right)$. Clearly, $S=\prod_{i \in I} S_{i}$. Let $\theta=\left\{\theta_{i}\right\}_{i \in I} \in$ $S$. Let $D_{S}(\theta)=\left\{\alpha=\left\{\alpha_{i}\right\}_{i \in I} \mid \alpha(M) \subseteq \theta(M)\right\}$ and $D_{S_{i}}\left(\theta_{i}\right)=\left\{f_{i} \in S_{i} \mid f_{i}\left(A_{i}\right) \subseteq\right.$ $\left.\theta_{i}\left(A_{i}\right)\right\}$ for each $i \in I$. It is not hard to see that $D_{S}(\theta)=\prod_{i \in I} D_{S_{i}}\left(\theta_{i}\right)$. Since each $A_{i}$ is $S G Q$-projective, there exists a right ideal $X_{i}$ of $S_{i}$ for each $i \in I$ such that $D_{S_{i}}\left(\theta_{i}\right)=\theta_{i} S_{i} \oplus X_{i}$. So $D_{S}(\theta)=\prod_{i \in I}\left(\theta_{i} S_{i}\right) \oplus \prod_{i \in I} X_{i}$. Let $X=\prod_{i \in I} X_{i}$. Note that $X$ is a right ideal of $S$ and $\prod_{i \in I}\left(\theta_{i} S_{i}\right)=\theta S$. Hence $D_{S}(\theta)=\theta S \oplus X$. Thus $M$ is $S G Q$-projective.

Now we characterize the class of rings $R$ for which every $R$-module is $S G Q$ projective as precisely that of the semisimple rings.

Theorem 3.1.15 The following are equivalent for an $R$-module $M$ :

(1) Every R-module is $S G Q$-projective.

(2) Every R-module is semi-Hopfian.

(3) $R$ is semisimple.

Proof. We only need to prove $(2) \Rightarrow(3)$. Let $X_{R}$ be a module. There exists an epimorphism $\varphi: F \longrightarrow X$ such that $F$ is free. Let $M_{R}=X \oplus F \oplus F \oplus \cdots$. Define the epimorphism

$$
\theta: M \longrightarrow M \text { by } \theta\left(x, f_{1}, f_{2}, \cdots, f_{n}, 0,0, \cdots\right)=\left(\varphi\left(f_{1}\right), f_{2}, f_{3}, \cdots, f_{n}, 0,0, \cdots\right) \text {. }
$$

Then $\operatorname{Ker} \theta=X \oplus \operatorname{Ker} \varphi \oplus 0 \oplus 0 \oplus \cdots$ is a direct summand of $M$. Hence $\operatorname{Ker} \varphi$ is a direct summand of $F$. So, $X$ is projective. Thus $R$ is semisimple.

\subsection{The Endomorphism Ring of SGQ-projective Modules}

Now denote;

$$
\begin{gathered}
\nabla=\{s \in S \mid \operatorname{Im} s \ll M\} \\
J(S)=\{s \in S \mid 1-s t \text { is right invertible for all } t \in S\}
\end{gathered}
$$




$$
\begin{aligned}
& \dot{\nabla}=\{s \in S \mid 1+s t \text { is epic for all } t \in S\} \\
& \operatorname{Tot}(M)=\operatorname{Tot}(S)=\{s \in S \mid s \text { is not pi }\}
\end{aligned}
$$

where $s$ is not pi means that there is no homomorphism $t \in S$ for which $s t=e$ (or equivalently, $t s=e$ ) is a nonzero idempotent in $S$ (see [7, Lemmas 14.1 and 14.3]).

It is clear that $J(S) \subseteq \dot{\nabla}$. Also $\nabla \subseteq \dot{\nabla}$. To show this take an element $s \in \nabla$. Since $\operatorname{Im} s \ll M$ and $M=\operatorname{Im} s+\operatorname{Im}(1+s t)$ for all $s, t \in S, M=\operatorname{Im}(1+s t)$. So $s \in \dot{\nabla}$. Another fact is $J(S) \subseteq \operatorname{Tot}(S)$. Assume that $s \in J(S)$ and $s \notin \operatorname{Tot}(S)$. There exists a homomorphism $t \in S$ for which $s t=e$ is a nonzero idempotent in $S$. And also $(1-s t)$ is right invertible. So $(1-s t) g=(1-e) g=1$. Then $e=0$, a contradiction.

Theorem 3.2.1 Let $M$ be an SGQ-projective module. Then

(1) $\nabla \subseteq J(S)=\dot{\nabla}$.

(2) If every proper submodule of $M$ is contained in a proper co- $M$-cyclic submodule of $M$, then $\nabla=J(S)$.

(3) If $S$ is local, then $J(S)=\dot{\nabla}=\operatorname{Tot}(S)=\{s \in S \mid s$ is not epic $\}$.

(4) If $S / \nabla$ is regular, then $J(S)=\nabla=\dot{\nabla}$.

(5) If $S / J(S)$ is regular, then $S / \nabla$ is regular if and only if $J(S)=\nabla$.

(6) If $M$ is hollow, then $S$ is local and $J(S)=\nabla=\dot{\nabla}=\operatorname{Tot}(S)=\{s \in S \mid s$ is not epic\}.

(7) For $s \in S$, if $M$ is hollow and $s$ is right invertible, then $s$ is invertible.

(8) $M$ is hollow if and only if $S$ is local and $M$ is $\pi$-projective.

Proof. (1) Let $s \in \nabla$ and $t \in S$. Since $\operatorname{Im} s \ll M$, then $\operatorname{Im} s t \ll M$. So $1-s t$ is epic and $1-s t \neq 0$. As $M$ is SGQ-projective $1-s t$ is right invertible from Lemma 3.1.6. Therefore $s \in J(S)$. Now let $s \in \dot{\nabla}$. Then for all $t \in S, 1+s t$ is epic. Since $M$ is $S G Q$-projective, $1+s t$ is right invertible for all $t \in S$. Thus $s \in J(S)$. 
(2) $\nabla \subseteq J(S)$ from (1). We need only to prove that $J(S) \subseteq \nabla$. Let $s \in J(S)$. Assume that $s \notin \nabla$. There exists a proper submodule $N$ of $M$ such that $M=$ $N+s(M)$. By hypothesis, there exists a proper co-M-cyclic submodule $K$ of $M$ such that $N \subseteq K$ and $M / K \cong T$ for some submodule $T$ of $M$. Therefore we have an epimorphism $\alpha: M \longrightarrow T$ with $\operatorname{Ker} \alpha=K$. Then $M=\operatorname{Ker} \alpha+s(M)$ and $T=\alpha(M)=\alpha s(M)$. Thus $\alpha \in D(\alpha s)$. Note that $\alpha s \neq 0$. Since $M$ is $S G Q$ projective, then there exists a right ideal $X$ of $S$ such that $D(\alpha s)=X \oplus(\alpha s) S$. Since $\alpha \in D(\alpha s), \alpha=x+\alpha s f$ for some elements $x \in X$ and $f \in S$. Since $s \in J(S)$, $1-s f$ is (right) invertible. Therefore $\alpha(1-s f)(1-s f)^{-1} s=\alpha s \in X \cap(\alpha s) S=0$, a contradiction. Hence $s \in \nabla$.

(3) It is clear that $J(S) \subseteq \operatorname{Tot}(S)$. Let $s \notin J(S)$. There exist an element $t \in S$ such that $1-s t$ is not right invertible. Since $S$ is local $s t$ is (right) invertible, so $s$ is invertible. Therefore $s \notin \operatorname{Tot}(S) . J(S)=\dot{\nabla}$ by (1). Now let $s \in J(S)$. Assume $s$ is epic. Since $M$ is SGQ-projective, $s$ is right invertible and $s S=S$, a contradiction. Therefore $J(S) \subseteq\{s \in S \mid s$ is not epic $\}$. The converse inclusion is easy.

(4) By (1), $\nabla \subseteq J(S)=\dot{\nabla}$. Let $s \in J(S)$. Since $S / \nabla$ is regular, there exists $\alpha \in S$ such that $s-s \alpha s=s(1-\alpha s) \in \nabla$. Since $(1-\alpha s)$ is invertible, $s \in \nabla$. This shows that $J(S) \subseteq \nabla$.

(5) By (4).

(6) By $[7,4.28(1)], S$ is local. By (3), the equalities $J(S)=\dot{\nabla}=\operatorname{Tot}(S)=\{s \in$ $S \mid s$ is not epic $\}$ hold. And from (1) $\nabla \subseteq J(S)$. Take an element $s \in S$ which is not epic. Then $\operatorname{Im} s \lesseqgtr M$. Since $M$ is hollow $\operatorname{Im} s \ll M$. Therefore $s \in \nabla$.

(7) Since $s$ has a right inverse, $s$ is epic. Since $M$ is hollow, $J(S)=\{s \in S \mid$ $s$ is not epic\} by (6). So $s \notin J(S)$. Hence $s$ is invertible.

(8) Suppose that $M$ is $\pi$-projective and $S$ is local. Let $N$ be a proper submodule of $M$. Assume $M=N+L$ for a submodule $L$ of $M$. Since $M$ is $\pi$-projective, there exists a homomorphism $f: M \longrightarrow M$ such that $f(M) \subseteq N$ and $(1-f)(M) \subseteq L$. Since $N$ is proper, $f$ cannot be epic. Therefore by (3), $f \in J(S)$. Thus $1-f$ is invertible. There exists a homomorphism $t \in S$ such that $(1-f) t=1$. Then $L \subseteq M=(1-f) t(M) \subseteq(1-f)(M) \subseteq L$. Hence $M=L$. 
Theorem 3.2.2 Let $M$ be a weakly supplemented $S G Q$-projective $\pi$-projective module. Then $S$ is regular if and only if $\nabla=0$.

Proof. Assume $S$ is regular. Then $J(S)=0$, and hence $\nabla=0$ by Theorem 3.2.1(1). Conversely, assume that $\nabla=0$. Let $0 \neq a \in S$. Then Ima is not small in $M$. Now there exists a proper submodule $K$ of $M$ such that $M=\operatorname{Im} a+K$ and $\operatorname{Im} a \cap K \ll M$. By hypothesis, $S=D(a)+D(K)$. Let $f \in D(a) \cap D(K)$. Then $\operatorname{Im} f \subseteq \operatorname{Im} a \cap K$ implies that $\operatorname{Im} f \ll M$. Thus $f \in \nabla$ and hence $f=0$. Therefore $S=D(a) \oplus D(K)$. Since $M$ is $S G Q$-projective, $S=a S \oplus X \oplus D(K)$ for some right ideal $X$ of $S$. It follows that $S$ is regular.

Corollary 3.2.3 Let $M$ be a weakly supplemented quasi-projective module. Then $S$ is regular if and only if $\nabla=0$.

Some dual characterizations of Theorem 3.2.1 exist in [56] and [61].

Following [46], a ring $R$ is called semiregular if $R / J(R)$ is regular and idempotents can be lifted modulo $J(R)$. By [54,42.11], $R$ is semiregular if and only if every finitely generated left ideal (right ideal) has a supplement in ${ }_{R} R\left(R_{R}\right)$, namely, it is left (right) f-semiperfect. Note that Lomp proved that for a ring $R, R / J(R)$ is regular if and only if every principal left (right) ideal of $R$ has a weak supplement in ${ }_{R} R\left(R_{R}\right)$ in [43, Proposition 3.18].

Theorem 3.2.4 Let $M$ be $S G Q$-projective. If $S$ is semiregular, then for every $s \in$ $S-J(S)$, there exists a nonzero idempotent $\alpha \in S$ such that $s-\alpha s$ is not epic.

Proof. Let $s \in S-J(S)$. Because $S / J(S)$ is regular, there exists an element $t+J(S)$ in $S / J(S)$ such that sts $-s \in J(S)$. Since $s t+J(S)$ is a nonzero idempotent in $S / J(S)$, there exists a nonzero idempotent $\alpha \in S$ such that $\alpha-s t \in J(S)$. Thus $s-\alpha s \in J(S)$. Since $M$ is $S G Q$-projective, $s-\alpha s$ cannot be epic.

Lemma 3.2.5 Let $M$ and $N$ be two modules. Then for every $\alpha: M \longrightarrow N$, Im $\alpha$ is a direct summand of $N$ if and only if $\sum_{i=1}^{k} \operatorname{Im} \alpha_{i}$ is a direct summand of $N$ for any finite set $\left\{\alpha_{1}, \cdots, \alpha_{k}\right\} \subseteq \operatorname{Hom}(M, N)$. 
Proof. See the proof of $(2) \Rightarrow(3)$ of [47, Theorem 4].

Theorem 3.2.6 The following are equivalent for a module $M$ :

(1) $S$ is regular.

(2) for every $\alpha \in S$, Im $\alpha$ is a direct summand of $M$ and $M$ is semi-projective.

(3) for every $\alpha \in S$, Im $\alpha$ is a direct summand of $M$ and $M$ is $S G Q$-projective.

(4) $\sum_{i=1}^{k} \operatorname{Im} \alpha_{i}$ is a direct summand of $M$ for any finite set $\left\{\alpha_{1}, \cdots, \alpha_{k}\right\} \subseteq S$ and $M$ is semi-projective.

(5) $\sum_{i=1}^{k}$ Im $\alpha_{i}$ is a direct summand of $M$ for any finite set $\left\{\alpha_{1}, \cdots, \alpha_{k}\right\} \subseteq S$ and $M$ is $S G Q$-projective.

(6) for every $\alpha \in S$, Im $\alpha$ is a direct summand of $M$ and $M$ is $\left(D_{2}\right)$.

(7) $\sum_{i=1}^{k} \operatorname{Im} \alpha_{i}$ is a direct summand of $M$ for any finite set $\left\{\alpha_{1}, \cdots, \alpha_{k}\right\} \subseteq S$ and $M$ is $\left(D_{2}\right)$.

Proof. $(1) \Rightarrow(2)$ By Corollary 2.1.12 and [54, 37.7].

$(2) \Rightarrow(3)$ and $(4) \Rightarrow(5) \Rightarrow(3)$ are clear.

(3) $\Rightarrow(1)$ Let $\alpha \in S$. Then $M=\operatorname{Im} \alpha \oplus N$ for some submodule $N$ of $M$. Clearly, $S=D(\operatorname{Im} \alpha) \oplus D(N)$. Since $M$ is $S G Q$-projective, $D(\operatorname{Im} \alpha)=\alpha S \oplus X$ for some right ideal $X$ of $S$. Hence $\alpha S$ is a direct summand of $S$. Thus $S$ is regular by [54, 3.10].

$(2) \Rightarrow(4)$ By Lemma 3.2.5.

$(1) \Leftrightarrow(6) \Leftrightarrow(7)$ By $[47$, Corollary 5].

Corollary 3.2.7 Let $M$ be any module such that for every $\alpha \in S$, Im $\alpha$ is a direct summand of $M$ (namely, $M$ is dual Rickart according to [42]). Then the following are equivalent:

(1) $S$ is regular.

(2) $M$ is semi-projective.

(3) $M$ is $S G Q$-projective.

(4) $M$ is $\left(D_{2}\right)$.

Note that any $S G Q$-projective module need not be dual Baer and any dual Baer module need not be $S G Q$-projective (see [34, Example 2.18] and Example 3.1.10). 


\section{A STUDY ON $\mathcal{K}$-PROJECTIVITY AND $\mathcal{K}^{*}$-PROJECTIVITY}

Given two $R$-modules $M$ and $N, N$ is called epi $M$-projective if, for any submodule $A$ of $M$, every epimorphism from $N$ to $M / A$ can be lifted to a homomorphism from $N$ to $M . N$ is called epi-projective if $N$ is epi-N-projective (see [7] and [32]). Let $M$ and $N$ be two $R$-modules. Remember that $N$ is $M$ - $\mathcal{K}$-projective if every homomorphism from $N$ to $M / A$, where $A$ is any co- $M$-cyclic submodule of $M$, can be lifted to a homomorphism from $N$ to $M$. We say that $M$ is quasi $\mathcal{K}$-projective if it is


modules as follows: Let $M$ and $N$ be two $R$-modules. $N$ is called epi $M$ - $\mathcal{K}$-projective if every epimorphism from $N$ to $M / A$ with $A$ any co- $M$-cyclic submodule of $M$ can be lifted to a homomorphism from $N$ to $M$. We will say that $M$ is quasi-epi $\mathcal{K}$-projective if it is epi $M$ - $\mathcal{K}$-projective. Note that $N$ is (epi) $M$ - $\mathcal{K}$-projective iff for every (epimorphism) homomorphism $f$ from $N$ to $A$ with $A \leq M$ and any epimorphism $\alpha$ from $M$ to $A$ there exists a homomorphism $g$ from $N$ to $M$ such that $\alpha g=f$.

In the definitions above, it should be noted that we may consider the case when $M / A$ to be isomorphic to a coclosed submodule of $M$. Namely, we will say that $N$ is (epi) $M-\mathcal{K}^{*}$-projective if every (epimorphism) homomorphism from $N$ to $M / A$ with $M / A$ isomorphic to a coclosed submodule of $M$ can be lifted to a homomorphism from $N$ to $M$. Note that $N$ is (epi) $M-\mathcal{K}^{*}$-projective iff for every (epimorphism) homomorphism $f$ from $N$ to $A$ with $A$ a coclosed submodule of $M$ and any epimorphism $\alpha$ from $M$ to $A$ there exists a homomorphism $g$ from $N$ to $M$ such that $\alpha g=f$. Similarly, we will say that $M$ is quasi(-epi) $\mathcal{K}^{*}$-projective if it is (epi) $M-\mathcal{K}^{*}$-projective. Note that quasi $\mathcal{K}^{*}$-projective modules were studied in [29] as $G Q$-projective modules and in [30] as modules satisfying $\left(T_{1}\right)$. A ring $R$ is called right $V$-ring if every right $R$-module has the zero Jacobson radical. A ring is a right $V$-ring if and only if every simple module is injective. In [15, Proposition 2.1], it is proved that a ring $R$ is a right $V$-ring if and only if for any $R$-module $M$, every submodule is coclosed in $M$. By this Proposition, if $R$ is a right $V$-ring, then (epi) $\mathcal{K}$-projectivity and (epi) $\mathcal{K}^{*}$-projectivity of any module $N$ are the same notions. 
In this section, we investigate the connections between the notions of epi projectivity, epi $\mathcal{K}$-projectivity and epi $\mathcal{K}^{*}$-projectivity, firstly. Then we show that if $M_{1} \oplus M_{2}$ is quasi-epi $\mathcal{K}\left(\mathcal{K}^{*}\right)$-projective, then $M_{1}$ and $M_{2}$ are relatively $\mathcal{K}\left(\mathcal{K}^{*}\right)$-projective. Later, we characterize semisimple rings in terms of quasi-epi $\mathcal{K}$-projective and quasi-epi $\mathcal{K}^{*}$-projective modules. Finally, we investigate the connections between the quasi(epi) $\mathcal{K}^{*}$-projective modules and the Hopfian modules.

\subsection{Properties of epi- $\mathcal{K}\left(\mathcal{K}^{*}\right)$-projectivity}

Example 4.1.1 Let $R$ be a local artinian ring with radical $W$ such that $W^{2}=0$, $Q=R / W$ is commutative, $\operatorname{dim}\left({ }_{Q} W\right)=2$ and $\operatorname{dim}\left(W_{Q}\right)=1$. Let $S=R / W$, the simple $R$-module.

(1) Consider the indecomposable injective right $R$-module $U=[(R \oplus R) / D]_{R}$ with $D=\{(u r,-v r) \mid r \in R\}$, where $W=R u+R v$. U has length 3 and is 2generated. On the other hand, $U$ has only two coclosed submodules, $U$ and any cyclic submodule with length 2 (which is isomorphic to $\mathrm{R}$ ). So if $U / N \cong X$ and $X$ is coclosed in $U$, then $N=0$. Therefore $U$ is quasi $\mathcal{K}^{*}$-projective.

(2) Consider the right $R$-module $M=U \oplus S$. Let $C$ be any cyclic submodule of $U$ with length 2 . Let $N=C \oplus S$. Then $M / N=(U \oplus S) /(C \oplus S) \cong U / C \cong S$. Let $f: M \longrightarrow M / N$ be a homomorphism with $\operatorname{Ker} f=U$. Then $f$ cannot be lifted to an endomorphism of $M$. Therefore $M$ is not quasi $\mathcal{K}^{*}$-projective.

Note that we have;

epi $M-$ projective $\Rightarrow$ epi $M-\mathcal{K}-$ projective $\Rightarrow$ epi $M-\mathcal{K}^{*}-$ projective.

The following example shows that an epi $M-\mathcal{K}^{*}$-projective module need not be epi $M$-projective.

Example 4.1.2 Let $M_{\mathbb{Z}}=\mathbb{Q} \oplus \mathbb{Z}^{(\mathbb{N})}$. $\mathbb{Q}_{\mathbb{Z}}$ is epi $\mathbb{Z}^{(\mathbb{N})}-\mathcal{K}^{*}$ (also epi $\left.\mathbb{Z}^{(\mathbb{N})}-\mathcal{K}\right)$-projective since every homomorphism from $\mathbb{Q}_{\mathbb{Z}}$ to $A$, where $A$ is any submodule of $\mathbb{Z}^{(\mathbb{N})}$, is zero. But it is not epi $\mathbb{Z}^{(\mathbb{N})}$-projective. Suppose that $Q_{\mathbb{Z}}$ is epi $\mathbb{Z}^{(\mathbb{N})}$-projective. Then it is easy to see that every epimorphism from $\mathbb{Z}^{(\mathbb{N})}$ to $Q_{\mathbb{Z}}$ splits. But it is a contradiction, since the obvious epimorphism from $\mathbb{Z}^{(\mathbb{N})}$ to $Q_{\mathbb{Z}}$ does not split. 
Proposition 4.1.3 1. Let $C$ be a co-M-cyclic submodule of $M$. If $M / C$ is epi $M-\mathcal{K}$-projective, then $C$ is a direct summand of $M$.

2. Let $C$ be a submodule of $M$ with $M / C \cong D$ and $D$ a coclosed submodule of $M$. If $M / C$ is epi $M-\mathcal{K}^{*}$-projective, then $C$ is a direct summand of $M$.

Proof. Straightforward.

Proposition 4.1.4 Let $B$ be a direct summand of $A$ and $N$ a direct summand of $M$.

1. If $A$ is epi $M$-K-projective, then $B$ is epi $N$-K-projective.

2. If $A$ is epi $M-\mathcal{K}^{*}$-projective, then $B$ is epi $N-\mathcal{K}^{*}$-projective.

Proof. Let $A=B \oplus B^{\prime}$ and $M=N \oplus N^{\prime}$.

1. Let $T$ be any submodule of $N$ and $\alpha: N \longrightarrow T$ and $f: B \longrightarrow T$ any epimorphisms. Consider the projection maps $\pi_{N}: M \longrightarrow N$ and $\pi_{B}: A \longrightarrow B$. Then, since $A$ is epi $M$ - $\mathcal{K}$-projective, there exists a homomorphism $g: A \longrightarrow$ $M$ such that $\alpha \pi_{N} g=f \pi_{B}$. It is easy to see that the homomorphism $\pi_{N}\left(g_{\mid B}\right)$ lifts $f$.

2. Let $T$ be any coclosed submodule of $N$ and $\alpha: N \longrightarrow T$ and $f: B \longrightarrow T$ any epimorphisms. By Proposition 1.2.25, $T$ is a coclosed submodule of $M$. Now we can apply the same proof in (1).

Corollary 4.1.5 Any direct summand of a quasi-epi $\mathcal{K}\left(\mathcal{K}^{*}\right)$-projective module is again quasi-epi $\mathcal{K}\left(\mathcal{K}^{*}\right)$-projective.

Proposition 4.1.6 Any quasi-epi $\mathcal{K}^{*}$-projective module satisfies the condition $\left(D_{2}\right)$. In particular, every semi-projective module satisfies the condition $\left(D_{2}\right)$.

Proof. Let $A$ and $B$ be two submodules of a quasi-epi $\mathcal{K}^{*}$-projective module $M$ with $A$ a direct summand of $M$ and $M / B \cong A$. Now we will show that $B$ is a direct summand of $M$. By Proposition 4.1.4, $A$ is epi $M-\mathcal{K}^{*}$-projective and hence $M / B$ is epi $M-\mathcal{K}^{*}$-projective. By Proposition 4.1.3, $B$ is a direct summand of $M$. 
Theorem 4.1.7 Let $M$ be any module. Then $M$ is quasi-epi $\mathcal{K}^{*}$-projective and lifting if and only if it is discrete.

Proof. By Propositions 4.1.6 and [29, Lemma, 2.6].

Note that in [29, Theorem 2.7], D.Keskin proved that for a module $M, M$ is discrete if and only if $M$ is lifting and $G Q$-projective. Theorem 4.1.7 generalizes the Theorem 2.7 in [29].

Theorem 4.1.8 1. If $M_{1} \oplus M_{2}$ is quasi-epi $\mathcal{K}$-projective, then $M_{1}$ and $M_{2}$ are relatively $\mathcal{K}$-projective.

2. If $M_{1} \oplus M_{2}$ is quasi-epi $\mathcal{K}^{*}$-projective, then $M_{1}$ and $M_{2}$ are relatively $\mathcal{K}^{*}$ projective.

\section{Proof.}

1. Let $M_{1} \oplus M_{2}$ be quasi-epi $\mathcal{K}$-projective. We will show that $M_{1}$ is $M_{2}-\mathcal{K}$ projective. Let $A \leq M_{2}, \alpha: M_{2} \longrightarrow A$ be any epimorphism and $f: M_{1} \longrightarrow A$ any homomorphism. Define $g: M_{1} \oplus M_{2} \longrightarrow A$ by $g\left(a_{1}+a_{2}\right)=f\left(a_{1}\right)+\alpha\left(a_{2}\right)$ for $a_{1} \in M_{1}$ and $a_{2} \in M_{2}$. Then $g$ is an epimorphism. By Proposition 4.1.4, $M_{1} \oplus M_{2}$ is epi $M_{2}$ - $\mathcal{K}$-projective. Then $g$ can be lifted to a homomorphism $h: M_{1} \oplus M_{2} \longrightarrow M_{2}$. Let $\eta:=h_{\mid M_{1}}: M_{1} \longrightarrow M_{2}$ be the restriction of $h$ to $M_{1}$. It is easy to see that $\alpha \eta=f$.

2. By the same argument as the proof of (1).

Corollary 4.1.9 If $M_{1} \oplus M_{2}$ is quasi $\mathcal{K}\left(\mathcal{K}^{*}\right)$-projective, then $M_{1}$ and $M_{2}$ are relatively $\mathcal{K}\left(\mathcal{K}^{*}\right)$-projective.

Corollary 4.1.10 Let $M=\oplus_{i=1}^{n} M_{i}$ be quasi-epi $\mathcal{K}\left(\mathcal{K}^{*}\right)$-projective. Then $M_{i}$ is $M_{j}-\mathcal{K}\left(\mathcal{K}^{*}\right)$-projective for all distinct $i, j \in\{1, \ldots, n\}$.

Proof. By Proposition 4.1.4, Theorem 4.1.8 and induction.

Next we characterize semisimple rings in terms of quasi-epi $\mathcal{K}$-projective and quasi-epi $\mathcal{K}^{*}$-projective modules. 
Theorem 4.1.11 For a ring $R$ the following are equivalent:

1. The direct sum of any two quasi-epi $\mathcal{K}$-projective modules is quasi-epi $\mathcal{K}$ projective.

2. Every cyclic quasi-epi $\mathcal{K}$-projective module is projective.

3. The direct sum of any two quasi-epi $\mathcal{K}^{*}$-projective modules is quasi-epi $\mathcal{K}^{*}$ projective.

4. Every cyclic quasi-epi $\mathcal{K}^{*}$-projective module is projective.

5. $R$ is semisimple.

Proof. $(1) \Rightarrow(2)$ Let $M$ be a cyclic quasi-epi $\mathcal{K}$-projective module. Suppose $N=$ $M \oplus R$. By assumption, $N$ is quasi-epi $\mathcal{K}$-projective. Hence $M$ is epi $N$-K $\mathcal{K}$-projective by Proposition 4.1.4. Let $M=x R$ for some element $x \in M$. Then $M \cong R / I$ for some right ideal $I$ of $R$. Clearly, $(M \oplus R) /(M \oplus I) \cong M$. By Proposition 4.1.3, $M \oplus I$ is a direct summand of $N$, and hence $I$ is a direct summand of $R$. Thus $R / I$ is projective, so is $M$.

$(2) \Rightarrow(5)$ Let $M$ be a simple $R$-module. Since $M$ is cyclic and quasi-epi $\mathcal{K}$ projective, it is projective by assumption. Thus $R$ is semisimple.

$(5) \Rightarrow(1)$ Clear.

$(3) \Leftrightarrow(4) \Leftrightarrow(5)$ By the same argument.

\subsection{Connections Between the Quasi(-epi) $\mathcal{K}\left(\mathcal{K}^{*}\right)$-projective Modules and the Hopfian Modules}

A module $M$ is said to be co-Hopfian if every injective endomorphism $f: M \longrightarrow M$ is an isomorphism, (see [7]). An $R$-module $M$ is directly finite if it is not isomorphic to a proper direct summand of $M$. A ring $R$ is directly finite if for any elements $a, b \in R, a b=1$ implies $b a=1 . R_{R}$ is directly finite if and only if $R$ is directly finite, $($ see $[7])$.

Proposition 4.2.1 $\quad$ 1. Let $M$ be a quasi-epi $\mathcal{K}$-projective module and $N$ be a fully invariant small submodule of $M$ such that $N$ is a co-M-cyclic submodule of $M$. Then $M$ is Hopfian if and only if $M / N$ is Hopfian. 
2. Let $M$ be a quasi-epi $\mathcal{K}^{*}$-projective module and $N$ be a fully invariant small submodule of $M$ such that $M / N$ is isomorphic to a coclosed submodule of $M$. Then $M$ is Hopfian if and only if $M / N$ is Hopfian.

\section{Proof.}

1. Assume $M$ is Hopfian. Let $f: M / N \longrightarrow M / N$ be any surjective homomorphism and $\pi: M \longrightarrow M / N$ be the natural epimorphism. Since $M$ is quasi-epi $\mathcal{K}$-projective, there exists a homomorphism $h: M \longrightarrow M$ such that $\pi h=f \pi$. Then $M=h(M)+N$ and so $h$ is epic since $N$ is small in $M$. Thus $h$ is an isomorphism since $M$ is Hopfian. Now $h(N)=h^{-1}(N)=N$ since $N$ is fully invariant in $M$. Then $\operatorname{Ker}(f)=0$. Hence $f$ is an isomorphism. This means that $M / N$ is Hopfian.

Conversely, assume that $M / N$ is Hopfian. Let $f: M \longrightarrow M$ be any epimorphism. Since $N$ is fully invariant in $M, f(N) \subseteq N$ and hence we have the homomorphism $\bar{f}: M / N \longrightarrow M / N$ defined by $\bar{f}(m+N)=f(m)+N$ $(m \in M)$. Clearly $\bar{f}$ is an epimorphism. Then by hypothesis, $\bar{f}$ is an isomorphism. $\operatorname{Ker}(\bar{f})=f^{-1}(N) / N=0$ implies that $\operatorname{Ker}(f) \subseteq f^{-1}(N)=N$. Since $N$ is small in $M, \operatorname{Ker}(f)$ is small in $M$. On the other hand, since $f: M \longrightarrow M$ is an epimorphism and $M$ is quasi-epi $\mathcal{K}$-projective, $\operatorname{Ker}(f)$ is a direct summand of $M$ by Proposition 4.1.6. Thus $\operatorname{Ker}(f)=0$. This means that $M$ is Hopfian.

2. The same proof as (1).

Corollary 4.2.2 Let $M$ be a quasi $\mathcal{K}\left(\mathcal{K}^{*}\right)$-projective module and $N$ be a fully invariant small submodule of $M$ such that $M / N$ is isomorphic to a (coclosed) submodule of $M$. Then $M$ is Hopfian if and only if $M / N$ is Hopfian.

Lemma 4.2.3 [45, Proposition 1.25] An $R$-module $M$ is directly finite if and only if $f g=1$ implies that $g f=1$ for any $f, g: M \longrightarrow M$.

Proposition 4.2.4 Let $M$ be a quasi-epi $\mathcal{K}^{*}$-projective directly finite module. Then $M$ is Hopfian. 
Proof. Let $f$ be any epimorphism from $M$ to $M$ and $1: M \longrightarrow M$ be the identity map. Since $M$ is quasi-epi $\mathcal{K}^{*}$-projective, there exists a homomorphism $g: M \longrightarrow M$ such that $f g=1$. By Lemma 4.2.3, $g f=1$ which shows that $f$ is a monomorphism. Thus $M$ is Hopfian.

Corollary 4.2.5 Let $M$ be a quasi $\mathcal{K}^{*}$-projective directly finite module. Then $M$ is Hopfian.

Proposition 4.2.6 Let $M$ be a quasi-epi $\mathcal{K}^{*}$-projective co-Hopfian module. Then $M$ is Hopfian.

Proof. Since $M$ is co-Hopfian, it is directly finite. Thus by Proposition 4.2.4, $M$ is Hopfian.

Corollary 4.2.7 Let $M$ be a quasi $\mathcal{K}^{*}$-projective co-Hopfian module. Then $M$ is Hopfian.

Note that dualizations of the above results can be found in [4]. 


\section{MODULES OVER FORMAL TRIANGULAR MATRIX RINGS}

At the beginning of this section, we will first explain the notions that we will be adopting. The following backgrounds were taken from [24]. For more details on formal triangular matrix rings we refer to [24], [22], [23], [5], [39], [37], [38].

For any left $B$, right $A$ bimodule ${ }_{B} M_{A}$ we write $T$ for the formal triangular matrix $T=\left[\begin{array}{cc}A & 0 \\ M & B\end{array}\right]$. Let $\Omega$ denote the category whose objects are triples $(X, Y)_{f}$ where $X \in \operatorname{Mod}-A, Y \in \operatorname{Mod}-B$ and $f: Y \otimes_{B} M \longrightarrow X$ is a map in $\operatorname{Mod}-A$. If $(X, Y)_{f}$ and $(U, V)_{g}$ are two objects in $\Omega$, then the morphisms from $(X, Y)_{f}$ to $(U, V)_{g}$ in $\Omega$ are pairs $\left(\varphi_{1}, \varphi_{2}\right)$ where $\varphi_{1}: X \longrightarrow U$ is an $A$-homomorphism, $\varphi_{2}: Y \longrightarrow V$ is a $B$-homomorphism satisfying the condition $\varphi_{1} f=g\left(\varphi_{2} \otimes 1_{M}\right)$. It is well known from [21] that the category $\Omega$ is equivalent to the category Mod- $T$. The right $T$-module corresponding to the triple $(X, Y)_{f}$ is the additive group $X \oplus Y$ with the right action given by

$$
(x, y)\left[\begin{array}{rr}
a & 0 \\
m & b
\end{array}\right]=(x a+f(y \otimes m), y b) .
$$

Then we write $(X \oplus Y)_{T}$ for this right $T$-module. It not only depends on $X$ and $Y$ but also on $f$.

Furthermore, if $\left(\varphi_{1}, \varphi_{2}\right):(X, Y)_{f} \longrightarrow(U, V)_{g}$ is a map in $\Omega$, the associated $T$ homomorphism $\varphi:(X \oplus Y)_{T} \longrightarrow(U \oplus V)_{T}$ is given by $\varphi(x, y)=\left(\varphi_{1}(x), \varphi_{2}(y)\right)$ for any $x \in X$ and $y \in Y$. It is clear that $\varphi$ is injective (resp. surjective) if and only if $\varphi_{1}: X \longrightarrow U, \varphi_{2}: Y \longrightarrow V$ are injective (resp. surjective). It is convenient to view such triples as $T$-modules and the morphisms between them as $T$-homomorphisms. Here we should note that the $T$-module $T_{T}$ corresponds to $(A \oplus M, B)_{f}$, where $f$ is the $A$-homomorphism $B \otimes M \longrightarrow A \oplus M$ given by $f(b \otimes m)=(0, b m)$.

Let $(X, Y)_{f} \in \operatorname{Obj}(\Omega)$ and $(X \oplus Y)_{T}$ be the associated right $T$-module. Under the right $T$-action on $X \oplus Y$ we have $(0 \oplus Y)\left[\begin{array}{rr}0 & 0 \\ M & 0\end{array}\right]=(f(Y \otimes M), 0)$. In general the submodule $f(Y \otimes M)$ of $X_{A}$ is denoted by $Y M$. Now consider $Y^{\prime} \leq$ $Y_{B}$ and let $j_{2}: Y^{\prime} \longrightarrow Y$ denote the inclusion map. Then $\left(0 \oplus Y^{\prime}\right)\left[\begin{array}{rr}0 & 0 \\ M & 0\end{array}\right]=$ 
$\left(f\left(j_{2} \otimes 1_{M}\right)\left(Y^{\prime} \otimes M\right), 0\right)$. In general, the submodule $f\left(j_{2} \otimes 1_{M}\right)\left(Y^{\prime} \otimes M\right)$ of $X_{A}$ is denoted by $Y^{\prime} M$. Let $X^{\prime} \leq X_{A}$ satisfy $Y^{\prime} M \subseteq X^{\prime}$. Writing $f^{\prime}$ for $f\left(j_{2} \otimes 1_{M}\right)$ and denoting the inclusion $X^{\prime} \longrightarrow X$ by $j_{1}$ we see that $\left(X^{\prime}, Y^{\prime}\right)_{f^{\prime}} \in \operatorname{Obj}(\Omega)$ and $\left(j_{1}, j_{2}\right):\left(X^{\prime}, Y^{\prime}\right)_{f^{\prime}} \longrightarrow(X, Y)_{f}$ is a map in $\Omega$ realizing $\left(X^{\prime} \oplus Y^{\prime}\right)_{T}$ as a $T$-submodule of $(X \oplus Y)_{T}$. Therefore when we take a submodule $\left(X^{\prime} \oplus Y^{\prime}\right)_{T}$ of $(X \oplus Y)_{T}$ we have $X^{\prime} \leq X_{A}, Y^{\prime} \leq Y_{B}, f\left(j_{2} \otimes 1_{M}\right)\left(Y^{\prime} \otimes M\right) \leq X^{\prime}$. The map $f^{\prime}: Y^{\prime} \otimes M \longrightarrow X^{\prime}$ is completely determined; it has to be $f\left(j_{2} \otimes 1_{M}\right)$.

Let $X^{\prime \prime}\left(\right.$ resp. $\left.Y^{\prime \prime}\right)$ be a quotient of $X_{A}\left(\right.$ resp. $Y_{B}$ ) with $\eta_{1}: X \longrightarrow X^{\prime \prime}$ (resp. $\left.\eta_{2}: Y \longrightarrow Y^{\prime \prime}\right)$ the canonical maps. Let $\operatorname{Ker} \eta_{1}=X^{\prime}$ and $\operatorname{Ker} \eta_{2}=Y^{\prime}$. Assume that $Y^{\prime} M \leq X^{\prime}$. Let $j_{1}: X^{\prime} \longrightarrow X, j_{2}: Y^{\prime} \longrightarrow Y$ be the inclusion maps. Clearly we have the $A$-homomorphism $f^{\prime \prime}: Y^{\prime \prime} \otimes M \longrightarrow X^{\prime \prime}$ rendering the following diagram commutative

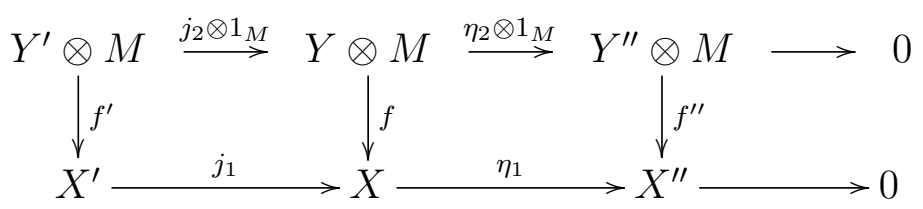

In this diagram $f^{\prime}=f\left(j_{2} \otimes 1_{M}\right)$ and the rows are exact. Also it is clear that $\left(\eta_{1}, \eta_{2}\right):(X, Y)_{f} \longrightarrow\left(X^{\prime \prime}, Y^{\prime \prime}\right)_{f^{\prime \prime}}$ is a map in $\Omega$ realizing $\left(X^{\prime \prime} \oplus Y^{\prime \prime}\right)_{T}$ as a quotient of $(X \oplus Y)_{T}$. The kernel of the associated T-homomorphism $\eta:(X \oplus Y)_{T} \longrightarrow$ $\left(X^{\prime \prime} \oplus Y^{\prime \prime}\right)_{T}$ is precisely $\left(X^{\prime} \oplus Y^{\prime}\right)_{T}$. Now when we deal with a quotient $\left(X^{\prime \prime} \oplus Y^{\prime \prime}\right)_{T}$ of $(X \oplus Y)_{T}$ the $A$-homomorphism $f^{\prime \prime}: Y^{\prime \prime} \otimes M \longrightarrow X^{\prime \prime}$ is completely determined.

In 2000, in [24], A. Haghany and K. Varadarajan characterized uniform, hollow, finitely embedded, projective, generator or progenerator modules over $T$. They determined the Jacobson radical $\operatorname{Rad}(X \oplus Y)_{T}$ and the socle $\operatorname{Soc}(X \oplus Y)_{T}$ of $(X \oplus Y)_{T}$. Projective right ideals over $T$ are completely characterized in [19]. A. Haghany and K. Varadarajan described an explicit method to constract a dual basis for projective modules. Also they give the necessary and sufficient conditions for a $T$-module to admit a projective cover.

This section of dissertation is devoted to the study of modules over formal triangular matrix rings and the results focus on relative projectivity and lifting properties of modules. We prove that if a right $T$-module $(X \oplus Y)_{T}$ is lifting, then $(X / Y M)_{A}$ and $Y_{B}$ are lifting. We also prove that if a right $T$-module $(X \oplus Y)_{T}$ is quasi- 
projective, then $(X / Y M)_{A}$ and $Y_{B}$ are quasi-projective and if a right $T$-module $(X \oplus Y)_{T}$ has a quasi-projective cover, then $(X / Y M)_{A}$ and $Y_{B}$ have semi-projective covers.

\subsection{Lifting Modules Over $T$}

As an easy observation we can give the following:

Proposition 5.1.1 $\left(X^{\prime} \oplus Y^{\prime}\right)_{T}$ is a direct summand of $(X \oplus Y)_{T}$ if and only if $X_{A}=X^{\prime} \oplus X^{\prime \prime}, Y_{B}=Y^{\prime} \oplus Y^{\prime \prime}$ with $f\left(j_{2}^{\prime} \otimes 1_{M}\right)=f^{\prime}, f\left(Y^{\prime} \otimes M\right) \subseteq X^{\prime}$ and $f\left(j_{2}^{\prime \prime} \otimes 1_{M}\right)=f^{\prime \prime}, f\left(Y^{\prime \prime} \otimes M\right) \subseteq X^{\prime \prime}$ where $j_{2}^{\prime}: Y^{\prime} \longrightarrow Y, j_{2}^{\prime \prime}: Y^{\prime \prime} \longrightarrow Y$ are the inclusion maps.

The following proposition from [24] gives necessary and sufficient conditions for $\left(X^{\prime} \otimes Y^{\prime}\right)_{T}$ to be small $(X \oplus Y)_{T}$.

Proposition 5.1.2 ([24]) $\left(X^{\prime} \oplus Y^{\prime}\right)_{T}$ is small in $(X \oplus Y)_{T}$ if and only if $Y^{\prime}$ is small in $Y_{B}$ and $\eta\left(X^{\prime}\right)$ is small in $(X / f(Y \otimes M))_{A}$ where $\eta: X \longrightarrow(X / f(Y \otimes M))$ is the canonical quotient map.

Any module $N$ is called lifting if for any submodule $H$ of $N$, there exists a decomposition $N=N_{1} \oplus N_{2}$ such that $N_{1} \subseteq H$ and $N_{2} \cap H \ll N_{2}$. Now we will give a characterization of lifting modules over the ring $T$ :

Theorem 5.1.3 If the right $T$-module $(X \oplus Y)_{T}$ determined by $(X, Y)_{f}$ is lifting, then $(X / Y M)_{A}$ and $Y_{B}$ are lifting.

Proof. Assume that $(X \oplus Y)_{T}$ is lifting. Let $Y^{\prime} \leq Y_{B}$. Consider the submodule $\left(X \oplus Y^{\prime}\right)_{T}$ of $(X \oplus Y)_{T}$ with the $A$-homomorphism $f^{\prime}=f\left(j_{2} \otimes 1_{M}\right)$ such that $j_{2}$ : $Y^{\prime} \longrightarrow Y$ is the inclusion map. Since $(X \oplus Y)_{T}$ is lifting, there exists a decomposition $(X \oplus Y)_{T}=\left(H^{\prime} \oplus K^{\prime}\right)_{T} \oplus\left(H^{\prime \prime} \oplus K^{\prime \prime}\right)_{T}$ such that $\left(H^{\prime} \oplus K^{\prime}\right)_{T} \subseteq\left(X \oplus Y^{\prime}\right)_{T}$ and $\left(H^{\prime \prime} \oplus K^{\prime \prime}\right)_{T} \cap\left(X \oplus Y^{\prime}\right)_{T}=\left(H^{\prime \prime} \oplus\left(K^{\prime \prime} \cap Y^{\prime}\right)\right)_{T} \ll\left(H^{\prime \prime} \oplus K^{\prime \prime}\right)_{T}$. Assume that $\left(H^{\prime \prime} \oplus K^{\prime \prime}\right)_{T}$ and $\left(H^{\prime} \oplus K^{\prime}\right)_{T}$ associate with the objects $\left(H^{\prime \prime}, K^{\prime \prime}\right)_{f^{\prime \prime}}$ and $\left(H^{\prime}, K^{\prime}\right)_{f^{\prime}}$ in $\Omega$ such that $f^{\prime}=f\left(j_{2}^{\prime} \otimes 1_{M}\right), f^{\prime \prime}=f\left(j_{2}^{\prime \prime} \otimes 1_{M}\right)$, where $j_{2}^{\prime}: K^{\prime} \longrightarrow Y$ and $j_{2}^{\prime \prime}: K^{\prime \prime} \longrightarrow Y$ are the inclusion maps and $f\left(K^{\prime} \otimes M\right) \subseteq H^{\prime}$ and $f\left(K^{\prime \prime} \otimes M\right) \subseteq H^{\prime \prime}$. Now we have that 
$X=H^{\prime} \oplus H^{\prime \prime}, Y=K^{\prime} \oplus K^{\prime \prime}, K^{\prime} \leq Y^{\prime}$. By Proposition 5.1.2, $K^{\prime \prime} \cap Y^{\prime} \ll K^{\prime \prime}$. Thus $Y_{B}$ is lifting.

Now let $X^{\prime} / Y M$ be an $A$-submodule of $X / Y M$. Then $\left(X^{\prime} \oplus Y\right)_{T}$ is a submodule of $(X \oplus Y)_{T}$ with the homomorphism $f$. Then there is a decomposition $(X \oplus Y)_{T}=$ $\left(L_{1} \oplus K_{1}\right)_{T} \oplus\left(L_{2} \oplus K_{2}\right)_{T}$ such that $\left(L_{1} \oplus K_{1}\right)_{T} \subseteq\left(X^{\prime} \oplus Y\right)_{T},\left(L_{2} \oplus K_{2}\right)_{T} \cap\left(X^{\prime} \oplus Y\right)_{T}=$ $\left(\left(L_{2} \cap X^{\prime}\right) \oplus K_{2}\right)_{T} \ll\left(L_{2} \oplus K_{2}\right)_{T}$. Now $X=L_{1} \oplus L_{2}$ and $Y=K_{1} \oplus K_{2}$. Also by Proposition 5.1.2, $K_{2}=0$ (so $f\left(K_{2} \otimes M\right)=0$ ) and $L_{2} \cap X^{\prime} \ll L_{2}$. Then $X / Y M=$ $L_{1} / Y M \oplus\left(L_{2} \oplus Y M\right) / Y M, L_{1} / Y M \subseteq X^{\prime} / Y M$ and $\left[X^{\prime} \cap\left(L_{2} \oplus Y M\right)\right] / Y M=$ $\left[Y M \oplus\left(X^{\prime} \cap L_{2}\right)\right] / Y M \ll\left(L_{2} \oplus Y M\right) / Y M$. Thus $(X / Y M)_{A}$ is lifting.

A ring $R$ is called generalized uniserial ring if it is artinian serial ring (see [10]). In 1995, K. Oshiro and R. Wisbauer proved in [48, Corollary 2.5] that every right $R$-module is lifting if and only if $R$ is generalized uniserial ring with $J(R)^{2}=0$. Using this corollary, we can give a part of a well-known fact in the following:

Corollary 5.1.4 If $T$ is a generalized uniserial ring with $J(T)^{2}=0$, then $B$ is a generalized uniserial ring with $J(B)^{2}=0$.

Proof. Let $T$ is a generalized uniserial ring with $J(T)^{2}=0$. Then every right $T$ module is lifting from [48, Corollary 2.5]. Then every right $B$-module is lifting from Theorem 5.1.3. So $B$ is a generalized uniserial ring with $J(B)^{2}=0$ again from $[48$, Corollary 2.5].

Example 5.1.5 Let $R$ be a ring and $M$ a right $R$-module. Let $T=\left[\begin{array}{cc}R & 0 \\ M & \mathbb{Z}\end{array}\right]$. Consider the right $T$-module $V_{T}=(M \oplus \mathbb{Z})_{T}$ associated to the triple $(M, \mathbb{Z})_{f}$ where $f: \mathbb{Z} \otimes M \longrightarrow M$ defined by $n \otimes m \mapsto n m$ for all $n \in \mathbb{Z}$ and $m \in M$. Since $\mathbb{Z}$ is not lifting, $V_{T}$ is not lifting.

\subsection{Relative Projectivity of Modules Over $T$}

Let $V_{T}=(X \oplus Y)_{T}$ be a right $T$-module corresponds to $(X, Y)_{f}$ in $\Omega$. Then we can define the following $B$-homomorphism:

$\tilde{f}: Y \longrightarrow \operatorname{Hom}(M, X)$ given by $\tilde{f}(y)(m)=f(y \otimes m)$ for $y \in Y, m \in M$. 
If the right $T$-module $V_{T}=(X \oplus Y)_{T}$ corresponds to $(X, Y)_{f}$ in $\Omega$ and $\left(X^{\prime} \oplus Y^{\prime}\right)_{T}$ is a submodule of $(X \oplus Y)_{T}$ with the homomorphism $f^{\prime}=f\left(j_{2} \otimes 1_{M}\right)$ such that $j_{2}: Y^{\prime} \longrightarrow Y$ is the inclusion map and $Y^{\prime} M \subseteq X^{\prime}$, then we will have the $B$ homomorphism:

$\tilde{f}_{\mid Y^{\prime}}: Y^{\prime} \longrightarrow \operatorname{Hom}\left(M, X^{\prime}\right)$ given by $\tilde{f}_{\mid Y^{\prime}}\left(y^{\prime}\right)(m)=f\left(y^{\prime} \otimes m\right)$ for $y^{\prime} \in Y^{\prime}, m \in M$.

A. Haghany and K. Varadarajan give the complete description of the projective right T-modules in [24, Theorem 3.1]. Also in [22] A. Haghany and in [5] J. Chen and $\mathrm{X}$. Zang investigate the relatively injectivity of right $T$-modules. Now we investigate the relatively projectivity of right $T$-modules in the following two theorems.

Theorem 5.2.1 Let $V_{1}$ and $V_{2}$ be two right T-modules with $\left(X_{1}, Y_{1}\right)_{f_{1}},\left(X_{2}, Y_{2}\right)_{f_{2}}$ the corresponding triples. If $X_{2}$ is $X_{1}$-projective in Mod-A and $\tilde{f}_{1 \mid Y_{1}^{\prime}}$ is an isomorphism for every submodule $\left(X_{1}^{\prime} \oplus Y_{1}^{\prime}\right)_{T}$ of $V_{1}$, then $V_{2}$ is $V_{1}$-projective in Mod-T.

Proof. Take a quotient $V_{1}^{\prime \prime}=\left(X_{1}^{\prime \prime}, Y_{1}^{\prime \prime}\right)_{f_{1}^{\prime \prime}}$ of $V_{1}$. Then $X_{1}^{\prime \prime}=X_{1} / X_{1}^{\prime}, Y_{1}^{\prime \prime}=Y_{1} / Y_{1}^{\prime}$, $\eta_{1}: X_{1} \longrightarrow X_{1}^{\prime \prime}$ and $\eta_{2}: Y_{1} \longrightarrow Y_{1}^{\prime \prime}$ are the natural epimorphisms, $\left(X_{1}^{\prime}, Y_{1}^{\prime}\right)_{f_{1}^{\prime}}$ is a submodule of $V_{1}$ with the homomorphism $f_{1}^{\prime}=f_{1}\left(j_{2}^{\prime} \otimes 1_{M}\right)\left(j_{2}^{\prime}: Y_{1}^{\prime} \longrightarrow Y_{1}\right.$ is the inclusion map) and $f_{1}^{\prime \prime}: Y_{1}^{\prime \prime} \otimes M \longrightarrow X_{1}^{\prime \prime}$ is the $A$-homomorphism which makes the following diagram commutative:

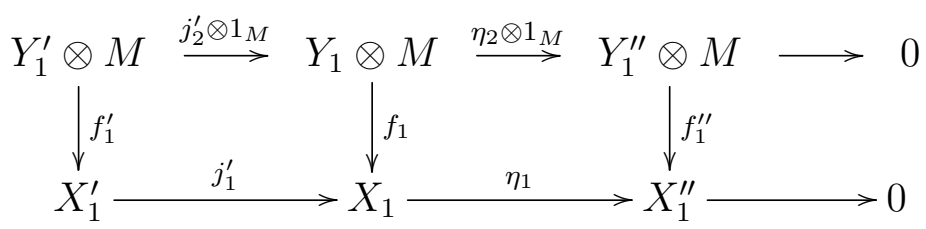

where $j_{1}^{\prime}: X_{1}^{\prime} \longrightarrow X_{1}$ is the inclusion map. Now the corresponding natural $T$ homomorphism $\eta$ from $V_{1}$ to $V_{1}^{\prime \prime}$ is the map $\left(\eta_{1}, \eta_{2}\right)$. Let $\sigma: V_{2} \longrightarrow V_{1}^{\prime \prime}$ be any $T$-homomorphism . Then $\sigma$ corresponds to the pair $\left(\sigma_{1}, \sigma_{2}\right)$ such that $\sigma_{1}: X_{2} \longrightarrow$ $X_{1}^{\prime \prime}$ is an $A$-homomorphism, $\sigma_{2}: Y_{2} \longrightarrow Y_{1}^{\prime \prime}$ is a $B$-homomorphism and $\sigma_{1} f_{2}=$ $f_{1}^{\prime \prime}\left(\sigma_{2} \otimes 1_{M}\right)$ and $\sigma\left(x_{2}, y_{2}\right)=\left(\sigma_{1}\left(x_{2}\right), \sigma_{2}\left(y_{2}\right)\right)$. Since $X_{2}$ is $X_{1}$-projective, there exists an $A$-homomorphism $\overline{\sigma_{1}}: X_{2} \longrightarrow X_{1}$ such that $\eta_{1} \overline{\sigma_{1}}=\sigma_{1}$. Now we want to define a $B$-homomorphism $\overline{\sigma_{2}}: Y_{2} \longrightarrow Y_{1}$ such that the pair $\left(\overline{\sigma_{1}}, \overline{\sigma_{2}}\right)$ lifts $\sigma$ with the corresponding $T$-homomorphism $\bar{\sigma}$. Take any element $y_{2} \in Y_{2}$. Then we can define a homomorphism $\theta: M \longrightarrow X_{1}$ with $\theta(m)=\bar{\sigma}_{1} f_{2}\left(y_{2} \otimes m\right)$. Since $\tilde{f}_{1}$ is an isomorphism, 
there exists a unique $y_{1} \in Y_{1}$ such that $\tilde{f}_{1}\left(y_{1}\right)=\theta$. Now let $\overline{\sigma_{2}}\left(y_{2}\right)=y_{1}$. Clearly $\overline{\sigma_{2}}$ is a $B$-homomorphism. Let $y_{2} \in Y_{2}$ and $m \in M$. Then $f_{1}\left(\overline{\sigma_{2}} \otimes 1_{M}\right)\left(y_{2} \otimes m\right)=f_{1}\left(\overline{\sigma_{2}}\left(y_{2}\right) \otimes\right.$ $m)=f_{1}\left(y_{1} \otimes m\right)=\tilde{f}_{1}\left(y_{1}\right)(m)=\theta(m)=\overline{\sigma_{1}} f_{2}\left(y_{2} \otimes m\right)$, where $\overline{\sigma_{2}}\left(y_{2}\right)=y_{1}$ and $\tilde{f}_{1}\left(y_{1}\right)=\theta$. Therefore $f_{1}\left(\overline{\sigma_{2}} \otimes 1_{M}\right)=\overline{\sigma_{1}} f_{2}$. Thus $\left(\overline{\sigma_{1}}, \overline{\sigma_{2}}\right):\left(X_{2}, Y_{2}\right)_{f_{2}} \longrightarrow\left(X_{1}, Y_{1}\right)_{f_{1}}$ is a morphism in $\Omega$ which corresponds to a $T$-homomorphism $\bar{\sigma}: V_{2} \longrightarrow V_{1}$, namely $\bar{\sigma}\left(x_{2}, y_{2}\right)=\left(\overline{\sigma_{1}}\left(x_{2}\right), \overline{\sigma_{2}}\left(y_{2}\right)\right)$. Now we should see that $\eta \bar{\sigma}=\sigma$. It is enough to show that $\eta_{2} \overline{\sigma_{2}}=\sigma_{2}$. Let $y_{2} \in Y_{2}$. Since $\sigma_{1} f_{2}=f_{1}^{\prime \prime}\left(\sigma_{2} \otimes 1_{M}\right)$, for all $m \in M,\left(\sigma_{1} f_{2}\right)\left(y_{2} \otimes\right.$ $m)=\sigma_{1}\left(f_{2}\left(y_{2} \otimes m\right)\right)=f_{1}^{\prime \prime}\left(\sigma_{2}\left(y_{2}\right) \otimes m\right)$, hence $\eta_{1} \overline{\sigma_{1}}\left(f_{2}\left(y_{2} \otimes m\right)\right)=f_{1}^{\prime \prime}\left(\sigma_{2}\left(y_{2}\right) \otimes m\right)$. Let $\sigma_{2}\left(y_{2}\right)=z_{1}+Y_{1}^{\prime}\left(z_{1} \in Y_{1}\right)$. On the other hand, $f_{1}^{\prime \prime}\left(\eta_{2} \otimes 1_{M}\right)=\eta_{1} f_{1}$. Thus, $f_{1}^{\prime \prime}\left(\left(\eta_{2} \otimes\right.\right.$ $\left.\left.1_{M}\right)\left(z_{1} \otimes m\right)\right)=\eta_{1} f_{1}\left(z_{1} \otimes m\right)=\eta_{1} \tilde{f}_{1}\left(z_{1}\right)(m)=\eta_{1} \bar{\sigma}_{1} f_{2}\left(y_{2} \otimes m\right)$, for all $m \in M$. Since $f_{1}\left(\overline{\sigma_{2}} \otimes 1_{M}\right)=\overline{\sigma_{1}} f_{2}, \eta_{1} \overline{\sigma_{1}} f_{2}\left(y_{2} \otimes m\right)=\eta_{1} f_{1}\left(\overline{\sigma_{2}} \otimes 1_{M}\right)\left(y_{2} \otimes m\right)=\eta_{1} f_{1}\left(\overline{\sigma_{2}}\left(y_{2}\right) \otimes m\right)=$ $\eta_{1} \tilde{f}_{1}\left(\overline{\sigma_{2}}\left(y_{2}\right)\right)(m)$, for all $m \in M$. Now $\eta_{1} \tilde{f}_{1}\left(z_{1}\right)(m)=\eta_{1} \tilde{f}_{1}\left(\overline{\sigma_{2}}\left(y_{2}\right)\right)(m)$, for all $m \in M$. This means that $\tilde{f}_{1}\left(z_{1}-\overline{\sigma_{2}}\left(y_{2}\right)\right)$ is an $A$-homomorphism from $M$ to $X_{1}^{\prime}$. Since $\tilde{f}_{1 \mid Y_{1}^{\prime}}$ is an isomorphism, there exists an element $y_{1}^{\prime} \in Y_{1}^{\prime}$ such that $\tilde{f}_{1 \mid Y_{1}^{\prime}}\left(y_{1}^{\prime}\right)=\tilde{f}_{1}\left(z_{1}-\bar{\sigma}_{2}\left(y_{2}\right)\right)$ and so $y_{1}^{\prime}=z_{1}-\overline{\sigma_{2}}\left(y_{2}\right)$. Thus $\sigma_{2}\left(y_{2}\right)=\eta_{2} \overline{\sigma_{2}}\left(y_{2}\right)$, namely $\sigma_{2}=\eta_{2} \overline{\sigma_{2}}$.

Note that in $[6,4.1 .1]$, it is proven that if $Y_{1}$ is $Y_{2}$-projective and $f_{1}: Y_{1} \otimes M \longrightarrow$ $X_{1}$ is an $A$-isomorphism, then $V_{1}$ is $V_{2}$-projective. Therefore we deduce that the converse of Theorem 5.2.1 may not be true. Namely there exist right $T$-modules $V_{1}$ and $V_{2}$ such that $V_{2}$ is $V_{1}$-projective but $X_{2}$ is not $X_{1}$-projective:

Example 5.2.2 Let $R$ be a ring and $M$ a right $R$-module such that ${ }_{\mathbb{Z}} M$ is torsionfree which is not quasi-projective. Again let $T=\left[\begin{array}{ll}R & 0 \\ M & \mathbb{Z}\end{array}\right]$ and consider the right $T$-module $V_{T}=(M \oplus \mathbb{Z})_{T}$ associated to the triple $(M, \mathbb{Z})_{f}$ where $f: \mathbb{Z} \otimes M \longrightarrow M$ defined by $n \otimes m \mapsto n m$ for all $n \in \mathbb{Z}$ and $m \in M$. Since $M$ is a torsion-free abelian group, $f$ is one-to-one and also epic. Therefore by $[6,4.1 .1], V_{T}$ is quasi-projective. On the other hand, $M$ is not quasi-projective.

Theorem 5.2.3 Let $V_{1}$ and $V_{2}$ be two right $T$-modules with $\left(X_{1}, Y_{1}\right)_{f_{1}},\left(X_{2}, Y_{2}\right)_{f_{2}}$ the corresponding triples. If $V_{2}$ is $V_{1}$-projective, then $Y_{2}$ is $Y_{1}$-projective and $X_{2} / f_{2}\left(Y_{2} \otimes\right.$ M) is $X_{1} / f_{1}\left(Y_{1} \otimes M\right)$-projective.

Proof. Let $\eta_{1}: Y_{1} \longrightarrow Y_{1} / K_{1}$ be the natural epimorphism and $\alpha_{1}: Y_{2} \longrightarrow Y_{1} / K_{1}$ be any $B$-homomorphism, where $K_{1} \leq Y_{1}$. Then we can construct the quotient 74 
$\left(0 \oplus Y_{1} / K_{1}\right)_{T}$ of $\left(X_{1} \oplus Y_{1}\right)_{T}$ with the following commutative diagram:

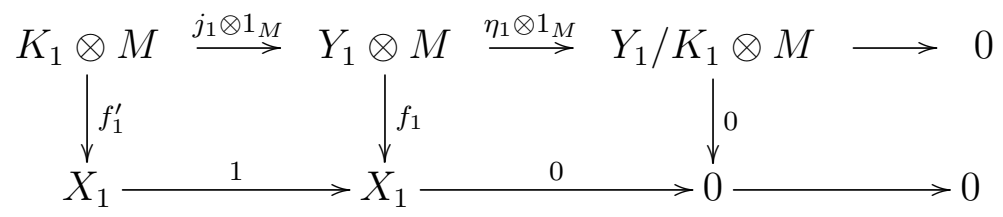

Now we can construct those morphisms in $\Omega$ :

$$
\left(0, \alpha_{1}\right):\left(X_{2}, Y_{2}\right)_{f_{2}} \longrightarrow\left(0, Y_{1} / K_{1}\right)_{0}
$$

and

$$
\left(0, \eta_{1}\right):\left(X_{1}, Y_{1}\right)_{f_{1}} \longrightarrow\left(0, Y_{1} / K_{1}\right)_{0}
$$

Thus we have the $T$-homomorphisms

$$
\alpha:\left(X_{2} \oplus Y_{2}\right)_{T} \longrightarrow\left(0 \oplus Y_{1} / K_{1}\right)_{T} \text { with } \alpha\left(x_{2}, y_{2}\right)=\left(0, \alpha_{1}\left(y_{2}\right)\right)
$$

and

$$
\eta:\left(X_{1} \oplus Y_{1}\right)_{T} \longrightarrow\left(0 \oplus Y_{1} / K_{1}\right)_{T} \text { with } \eta\left(x_{1}, y_{1}\right)=\left(0, \eta_{1}\left(y_{1}\right)\right)
$$

Note that $\eta$ is the natural epimorphism from $\left(X_{1} \oplus Y_{1}\right)_{T}$ to its quotient $\left(0 \oplus Y_{1} / K_{1}\right)_{T}$. Since $V_{2}$ is $V_{1}$-projective, there is a $T$-homomorphism $\beta: V_{2} \longrightarrow V_{1}$ such that $\eta \beta=\alpha$. Namely, there exists a $B$-homomorphism $\beta_{2}: Y_{2} \longrightarrow Y_{1}$ and an $A$-homomorphism $\beta_{1}: X_{2} \longrightarrow X_{1}$ such that $\beta_{1} f_{2}=f_{1}\left(\beta_{2} \otimes 1_{M}\right)$ and $\beta\left(x_{2}, y_{2}\right)=\left(\beta_{1}\left(x_{2}\right), \beta_{2}\left(y_{2}\right)\right)$. Thus $\eta_{1} \beta_{2}=\alpha_{1}$. Hence $Y_{2}$ is $Y_{1}$-projective.

Now consider the following diagram:

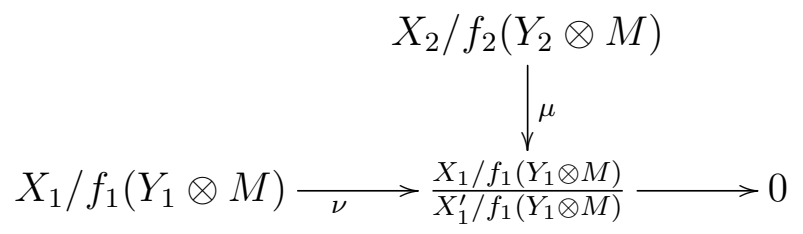

where $\nu$ is the natural epimorphism, $\mu$ is any $A$-homomorphism and $X_{1}^{\prime} / f_{1}\left(Y_{1} \otimes M\right)$ is a submodule of $X_{1} / f_{1}\left(Y_{1} \otimes M\right)$. Let $\gamma$ be the isomorphism from $\left(X_{1} / f_{1}\left(Y_{1} \otimes\right.\right.$ $M)) /\left(X_{1}^{\prime} / f_{1}\left(Y_{1} \otimes M\right)\right)$ to $X_{1} / X_{1}^{\prime}, \pi_{1}: X_{1} \longrightarrow X_{1} / f_{1}\left(Y_{1} \otimes M\right)$ and $\pi_{2}: X_{2} \longrightarrow$ $X_{2} / f_{2}\left(Y_{2} \otimes M\right)$ be the natural epimorphisms. It is clear that $\left(X_{1}^{\prime} \oplus Y_{1}\right)_{T}$ is a submodule of $V_{1}$ with $f_{1}^{\prime}=f_{1}$ and $\left(\left(X_{1} / X_{1}^{\prime}\right) \oplus 0\right)_{T}$ is a factor module of $V_{1}$ with $f_{1}^{\prime \prime}=0$, 
namely we have the following commutative diagram:

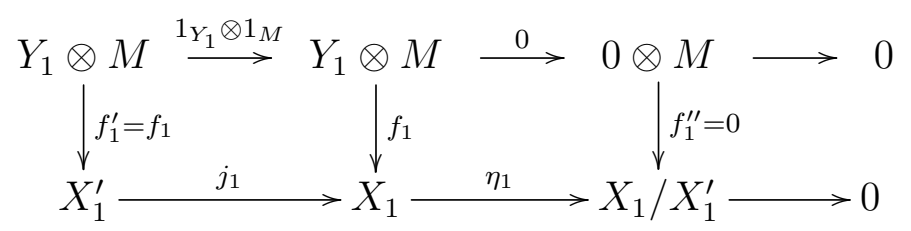

Now $\left(\gamma \mu \pi_{2}, 0\right):\left(X_{2}, Y_{2}\right)_{f_{2}} \longrightarrow\left(X_{1} / X_{1}^{\prime}, 0\right)_{0}$ is a $T$-homomorphism and $\left(\gamma \nu \pi_{1}, 0\right)$ : $\left(X_{1}, Y_{1}\right)_{f_{1}} \longrightarrow\left(X_{1} / X_{1}^{\prime}, 0\right)_{0}$ is a $T$-epimorphism. Since $V_{2}$ is $V_{1}$-projective, we have a $T$-homomorphism with the pair $\left(\mu_{1}, \mu_{2}\right):\left(X_{2}, Y_{2}\right)_{f_{2}} \longrightarrow\left(X_{1}, Y_{1}\right)_{f_{1}}$ which makes the following diagram commutative:

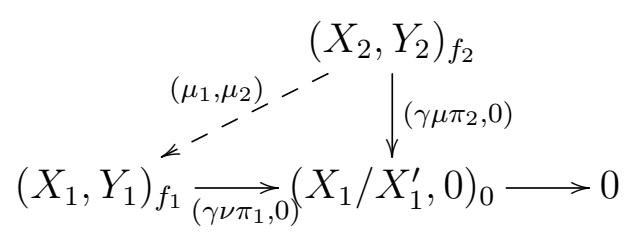

Note that we have the compositions $\mu_{1} f_{2}=f_{1}\left(\mu_{2} \otimes 1_{M}\right)$ and $\nu \pi_{1} \mu_{1}=\mu \pi_{2}$. Let us define the $A$-homomorphism $\bar{\mu}: X_{2} / f_{2}\left(Y_{2} \otimes M\right) \longrightarrow X_{1} / f_{1}\left(Y_{1} \otimes M\right)$ by $x_{2}+f_{2}\left(Y_{2} \otimes\right.$ $M) \mapsto \mu_{1}\left(x_{2}\right)+f_{1}\left(Y_{1} \otimes M\right)$. Since $\mu_{1} f_{2}=f_{1}\left(\mu_{2} \otimes 1_{M}\right), \bar{\mu}$ is well-defined and since $\nu \pi_{1} \mu_{1}=\mu \pi_{2}, \nu \bar{\mu}=\mu$. Therefore the following diagram is commutative:

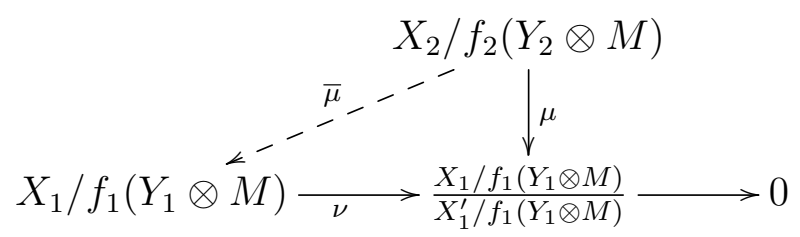

Therefore $X_{2} / f_{2}\left(Y_{2} \otimes M\right)$ is $X_{1} / f_{1}\left(Y_{1} \otimes M\right)$-projective.

Let $V_{1}$ and $V_{2}$ be two right $T$-modules with $\left(X_{1}, Y_{1}\right)_{f_{1}}$ and $\left(X_{2}, Y_{2}\right)_{f_{2}}$ the corresponding triples. If $V_{2}$ is $V_{1}$-projective, the relative projectivity of $Y_{2}$ with respect to $Y_{1}$ is also proven in $[6,4.1 .3]$ and under the assumption that $f_{1}\left(Y_{1} \otimes M\right) \leq_{d} X_{1}$, the relative projectivity of $X_{2} / f_{2}\left(Y_{2} \otimes M\right)$ with respect to $X_{1} / f_{1}\left(Y_{1} \otimes M\right)$ is proven in $[6$, 4.1.4]. In the above Theorem 5.2.3 we prove the relative projectivity of $X_{2} / f_{2}\left(Y_{2} \otimes M\right)$ with respect to $X_{1} / f_{1}\left(Y_{1} \otimes M\right)$ without the condition $f_{1}\left(Y_{1} \otimes M\right) \leq_{d} X_{1}$.

Corollary 5.2.4 If $(X \oplus Y)_{T}$ is quasi-projective, then $(X / Y M)_{A}$ and $Y_{B}$ are quasiprojective. 
Example 5.2.5 Let $R$ be a ring and $M$ be a right $R$-module. Consider the ring $T=\left[\begin{array}{ll}R & 0 \\ M & \mathbb{Z}\end{array}\right]$. Let $K$ be a nonzero submodule of $\mathbb{Q}_{\mathbb{Z}}$ with $K \nsubseteq \mathbb{Z}$ and $K \nsucceq \mathbb{Q}_{\mathbb{Z}}$. By Corollary 2.3.4, $K \oplus \mathbb{Z}$ is not semi-projective hence not quasi-projective over $\mathbb{Z}$. Then by Corollary 5.2.4, none of the right $T$-modules in the form $(X \oplus(K \oplus \mathbb{Z}))_{T}$ is quasi-projective, where $X$ is any right $R$-module.

Corollary 5.2.6 If $(X \oplus Y)_{T}$ has a quasi-projective cover, then $(X / Y M)_{A}$ and $Y_{B}$ have semi-projective covers.

Proof. Let $\varphi:(U \oplus V)_{T} \longrightarrow(X \oplus Y)_{T}$ be a quasi-projective cover of $(X \oplus Y)_{T}$. Assume that the objects $(U, V)_{g}$ and $(X, Y)_{f}$ in $\Omega$ determine the right $T$-modules $(U \oplus V)_{T}$ and $(X \oplus Y)_{T}$, respectively. Then there exist homomorphisms $\varphi_{1}: U_{A} \longrightarrow$ $X_{A}, \varphi_{2}: V_{B} \longrightarrow Y_{B}$ such that $\left(\varphi_{1}, \varphi_{2}\right):(U, V)_{g} \longrightarrow(X, Y)_{f}$ is a morphism in $\Omega$ with $\varphi_{1} g=f\left(\varphi_{2} \otimes 1_{M}\right)$ and $\left(\varphi_{1}(u), \varphi_{2}(v)\right)=\varphi(u, v)$. By [5, Theorem 2.4], the epimorphism $\varphi_{2}: V_{B} \longrightarrow Y_{B}$ has small kernel and we have the epimorphism $\overline{\varphi_{1}}$ : $U / V M \longrightarrow X / Y M$ with small kernel. Thus $(X / Y M)_{A}$ and $Y_{B}$ have semi-projective covers with the epimorphisms $\overline{\varphi_{1}}$ and $\varphi_{2}$, respectively by Corollary 5.2.4. 


\section{REFERENCES}

[1] Albu, T., Năstăsescu C., Relative Finiteness in Module Theory, Marcel Dekker, New York, Basel, 1984.

[2] Anderson, F. W., Fuller, K. R., Rings and Categories of Modules, Graduate Texts in Mathematics, Vol. 13, Springer-Verlag, New York, 1992.

[3] Bass, H., Finistic dimension and a homological generalization of semiprimary rings, Transactions of the American Mathematical Society, 95, 466-488, 1960.

[4] Chaturvedi, A. K., Pandeya, B. M., Gupta, A. J., Quasi-pseudo Principally Injective Modules, Algebra Colloquium, 16:3, 397-402, 2009.

[5] Chen, J., Zhang, X., On modules over formal triangular matrix rings, East-West Journal of Mathematics, 3(1), 69-77, 2001.

[6] Chen, Ju., Formal Triangular Matrix Rings and the Modules over Them, M.Sci. dissertation, Graduate School Of National University Of Defense Technology, November, 2006.

[7] Clark, J., Lomp, C., Vanaja, N., Wisbauer, R., Lifting Modules, Birkhäuser Verlag, Basel 2006.

[8] Colby R., R., Rutter, Jr. E. A., Generalizations of QF-3 rings, Transections of the Amererican Mathematical Society, Vol. 153, 1971.

[9] Dummit, D. S., Foote, R. M., Abstract Algebra, John Villey and Sons Inc., 2004.

[10] Faith, C., Algebra II Ring Theory, Springer-Verlag Berlin Heidelberg New York, 1976.

[11] Faticoni, T. G., On quasiprojective covers, Transactions of the American Mathematical Society, 278(1), 101-113, 1983. 
[12] Fuchs, L., Rangaswamy, K. M., Quasi-projective abelian groups, Bulletin de la Société Mathématique de France, 98, 5-8, 1970.

[13] Fuchs, L., Salce, L. , Module Over Non-Noetherian Domains, American Mathematical Society, 2000.

[14] Fuller, K., Hill, D. A., On quasiprojective modules via relative projectivity, Archive der Mathematik, 21, 369-373, 1970.

[15] Ganesan, L., Vanaja, N., Modules for which every submodule has a unique coclosure, Commmunications in Algebra, 30(5), 2355-2377, 2002.

[16] Golan, J., Characterizations of rings using quasi-projective modules, Israel Journal of Mathematics, 8, 34-38, 1970.

[17] Golan, J., Characterizations of rings using quasi-projective modules II, Proceedings of the American Mathematical Society, 28, 337-343 1971.

[18] Golan, J., Characterizations of rings using quasi-projective modules III, Proceedings of the American Mathematical Society, 31, 401-408, 1972.

[19] Goodearl, K. R., Ring Theory, (Nonsingular Rings and Modules), Marcel Dekker, Inc., New York and Bassel, 1976.

[20] Goodearl, K. R., Warfield, R. B., An Introduction to Noncommutative Noetherian Rings, London Mathematical Society Student Texts 16, Cambridge Univ. Press, Cambridge 1989.

[21] Green, E.L, On the representation theory of rings in matrix form, Pacific Journal of Mathematics, 100, 123-138, 1982.

[22] Haghany, A., Injectivity conditions over a formal triangular matrix rings, Archive der Mathematik, 78, 268-274, 2002.

[23] Haghany, A., Mazrooei, M., Vedadi, M. R., Pure projectivity and pure injectivity over formal triangular matrix rings, Journal of Algebra and Its Applications, 11(6), 2012. 
[24] Haghany, A., Varadarajan, K., Study of modules over formal triangular matrix rings, Journal of Pure and Applied Algebra, 147, 41-58, 2000.

[25] Haghany, A., Vedadi, M. R., Study of semi-projective retractable modules, Algebra Colloquium, 14(3), 489-496, 2007.

[26] Kalebog̃az, B., Keskin Tütüncü, D., A Study On Semi-projective Covers, Semi-projective Modules and Formal Triangular Matrix Rings, Palestine Journal of Mathematics, 3(1), 2014.

[27] Kaplansky, I., Modules over Dedekind rings and valuation rings, Transactions of the American Mathematical Society, 72, 327-340 1952.

[28] Kaye, S. M. , Ring theoretic properties of matrix rings, Canadian Mathematical Bulletin, 10, 365-374, 1967.

[29] Keskin, D., A generalization of quasi-projective modules, Far East Journal of Mathematical Sciences, 2(3), 483-488, 2000.

[30] Keskin, D., Discrete and quasi-discrete modules, Communications in Algebra, 30(11), 5273-5282, 2002.

[31] Keskin Tütüncü, D., Kalebog̃az, B., Smith, P. F., Direct sums of semi-projective modules, Colloquium Mathematicum, 127(1), 67-81

\section{2.}

[32] Keskin Tütüncü, D., Kuratomi, Y., On epi-projective modules, EastWest Journal of Mathematics, 10(1), 27-35, 2008.

[33] Keskin Tütüncü, D., Kuru, B., Some Generalizations of Quasi-projective Modules, Algebra Colloquium, accepted.

[34] Keskin Tütüncü, D., Tribak, R., On dual Baer modules, Glasgow Mathematical Journal, 52, 261-269, 2010.

[35] Koehler, A., Quasi-projective covers and direct sums, Proceedings of the American Mathematical Society, 24(4), 655-658, 1970. 
[36] Koehler, A., Rings for which every cyclic module is quasi-injective, Mathematische Annalen, 189, 311-316, 1970.

[37] Krylov, P. A., Injective modules over formal matrix rings, Siberian Mathematical Journal, 51(1), 72-77, 2010.

[38] Krylov, P. A., Tuganbaev, A. A., Modules over formal matrix rings, Journal of Mathematical Sciences, 171(2), 248-295, 2010.

[39] Krylov, P. A., Yu Yardykov, E., Projective and hereditary modules over rings of generalized matrices, Journal of Mathematical Sciences, 163(6), 709-719, 2009.

[40] Kumar, V., Gupta, A. J., Pandeya, B. M., Patel, M. K., M-SPProjective Modules, International Journal of Algebra, Vol. 5, no. 12, 563-568, 2011.

[41] Lam, T. Y., Lectures on Modules and Rings, Springer-Verlag, BerlinHeidelberg-New York, 1999.

[42] Lee, G., Rizvi, S. T., Roman, C., Dual rickart modules, Communications in Algebra, 39(11), 4036-4058, 2011, 4036-4058.

[43] Lomp, C., On semilocal modules and rings, Communications in Algebra, 27(4), 1921-1935, 1999.

[44] McConnell, J. C., Robson, J. C., Noncommutative Noetherian Rings, Wiley-Interscience, Chichester, 1987.

[45] Mohamed, S. H., Müller, B. J., Continuous and Discrete Modules, London Mathematical Society Lecture Series 147, Cambridge University Press, Cambridge, 1990.

[46] Nicholson, W. K., Semiregular modules and rings, Canadian Journal of Mathematics, 28, 1105-1120, 1976.

[47] Nicholson, W. K., Zhou, Y., Semiregular Morphisms, Communications in Algebra, 34, 219-233, 2006. 
[48] Oshiro, K., Wisbauer, R., Modules with every subgenerated module lifting, Osaka Journal of Mathematics, 32, 513-519, 1995.

[49] Rangaswamy, K. M., Abelian groups with endomorphic images of special types, Journal of Algebra, 6, 271-280,1967.

[50] Rangaswamy, K. M., Vanaja, N., Quasi Projectives in Abelian and Module Categories, Pacific Journal of Mathematics, 43 (1), 221-238, 1972.

[51] Rotman, J. J., An Introduction to Homological Algebra, Springer Science+Business Media, 2009.

[52] Tansee, H., Wongwai, S., A note on semi-projective modules, Kyungpook Mathematical Journal, 42, 369-380, 2002.

[53] Wang, X., Chen, J., On relatively semi-projective and direct-projective modules, Northeastern Mathematical Journal, 24(5), 409-422, 2008.

[54] Wisbauer, R., Foundations of Module and Ring Theory, Gordon and Breach, Philadelphia, 1991.

[55] Wisbauer, R., Modules and Algebras: Bimodule Structure On Group Actions and Algebras, Pitman Monographs 81, Longman, 1996.

[56] Wongwai, S., On the Endomorphism Ring of a Semi-injective Module, Acta Mathematica Universitatis Comenianae, Vol. LXXI, 1, 27-33, 2002.

[57] Wu, L. E. T., Jans, J. P., On quasi projectives, Illinois Journal of Mathematics, 11, 439-448, 1967.

[58] Xue, W., Characterizations of rings using direct-projective modules and direct-injective modules, Journal of Pure and Applied Algebra, 87, 99-104, 1993.

[59] Xue, W., Characterizations of semiperfect and perfect rings, Publicacions Matematiques, 40, 115-125, 1996. 
[60] Yousif, M., Amin, I., Ibrahim, Y., D3-Modules, Communications in Algebra, 42, 578-592, 2014.

[61] Zhu, Z., Zhang, X., AGQP-Injective Modules, International Journal of Mathematics and Mathematical Sciences (Hindawi Publishing Corporation), Article ID 469725, doi:10.1155, 2008. 


\section{Index}

$\left(D_{2}\right)$ condition, 9

$M$ - $\mathcal{K}$-projective module, 52,62

$M$-cyclic submodule, 19

$M$-projective module, 5

$M$-sprojective, 20

$X$-torsion, 15

$X$-torsion-free, 15

$\Omega$ category, 69

$\mathcal{U}$-generated, 4

$\pi$-projective module, 8

$\sum$-semi-projective module, 49

(epi) $M-\mathcal{K}^{*}$-projective module, 62

ample supplement submodule, 9

amply supplemented module, 9

annihilator of a module, 3

classical right quotient ring, 13, 31

co- $M$-cyclic submodule, 52

co-Hopfian module, 66

coclosed submodule, 9

cosmall submodule, 9

direct projective module, 21

directly finite module, 66

directly finite ring, 66

discrete module , 10

divisible module, 16

epi $M-\mathcal{K}$ projective module, 62

epi- $M$-projective module, 62 epi-projective module, 62

essential extension, 1

essential submodule, 1

extending module, 24

faithful module, 3

finite rank, 10

finitely supplemented module, 9

formal triangular matrix ring, 69

generator, 4

Goldie's Regular Element Lemma, 14

Goldie's Theorem, 15

hollow module, 1

Hopfian module, 54

indecomposable module, 2

injective hull, 10

injective module, 10

intrinsically projective module, 8, 18

Jacobson radical, 2

lifting module, 10, 71

local module, 2

multiplicative set, 10

nonsingular module, 3

PI-ring (Polynomial identity ring), 14

prime ideal, 3

prime ring, 3 
projective cover, 6,40

projective module, 5

quasi $\mathcal{K}$-projective module, 53, 62

quasi(-epi) $\mathcal{K}^{*}$-projective module, 62

quasi-discrete module, 10

quasi-epi $\mathcal{K}$-projective module, 62

quasi-projective cover, 40

quasi-projective module, 7

regular element, 10, 31

Rickart module, 23

right $V$-ring, 62

right annihilator of a ring, 14

right bounded, 14

right Goldie ring, 14

right hereditary ring, 7

right order, 13, 31

right Ore condition, 11, 31

right Ore domain, 13, 31

right Ore set, 11

right perfect ring, 6

right PP-ring, 7

right primitive ideal, 3

right primitive ring, 3

right quotient division ring, 13, 31

right quotient ring, 11

right ring of fractions, 11, 31

right semihereditary ring, 7

right T-nilpotent, 4

semi-Hopfian module, 29

semi-primary ring, 18 semi-projective cover, 41

semi-projective module, $8,18,20$

semiperfect ring, 6

semiprime ideal, 3

semiprime ring, 3

semiregular ring, 60

semisimple module, 2

semisimple ring, 2

SGQ-projective, 52, 53

simple module, 2

singular module, 3

singular submodule, 3

small submodule, 1

socle, 2

split exact sequence, 6

supplement submodule, 9

supplemented module, 9

the cardinal $g\left(M_{R}\right), 34$

the ideal $D(\alpha), 22$

the ideal $D(N), 22$

the subring $\mathcal{O}(X), 31$

torsion module, 16

torsion submodule, 16

torsion-free module, 16

uniform module, 1

von Neumann regular ring, 6

weak supplement submodule, 9

weakly supplemented module, 9 


\section{CURRICULUM VITAE}

\section{$\underline{\text { Credentials }}$}

First Name : Berke

Last Name : Kaleboğaz

Place of Birth : Ankara, Turkey

Marial Status : Married

E-mail $\quad$ : bkuru@hacettepe.edu.tr

Adress : Güzeltepe Mah., Hoşdere Cad., Halit Ziya Sok., 12-11, Çankaya-ANKARA

\section{Education}

High School : 1999-2002 Bartın Davut Firıncıoğlu Anatolian High School

BSc. : 2002-2003 Hacettepe University, Foreign Languages Academy, English Class

2003-2007 Hacettepe University, Faculty of Science Department of Mathematics.

MSc. : 2007-2009 Hacettepe University, Institute of Graduate Studies in Science, Department of Mathematics.

PhD. : 2009-2014 Hacettepe University, Institute of Graduate Studies in Science, Department of Mathematics.

\section{Foreign Languages}

English

\section{Work Experience}

2008- Research Assistant, Hacettepe University, Faculty of Science, Department of Mathematics. 


\section{Areas of Experience}

Non-commutative Algebra, Module and Ring Theory

\section{Projects and Budgets}

I was awarded a grant (2214-A-International Research Felllowship Programme) by The Scientific and Technological Research Council of Turkey (TUBITAK) for 6 months.

\section{$\underline{\text { Publications }}$}

1. Keskin Tütüncü D., Kuru B., Some Generalizations of Quasi-projective Modules, Algebra Colloquium, accepted.

2. Keskin Tütüncü D. , Kalebog̃az B. and Smith P. F., Direct sums of semiprojective modules, Colloquium Mathematicum, 127(1), 67-81 2012.

3. Kalebog̃az B. and Keskin Tütüncü D., A Study On Semi-projective Covers, Semi-projective Modules and Formal Triangular Matrix Rings, Palestine Journal of Mathematics(In special issue for International Conference on Algebra, Balikesir, Turkey), 3(1), 2014.

\section{Oral and Poster Presentations}

1. The First International Conference on Mathematics and Statistics, American University of Sharjah, Sharjah, U.A.E., 18-21 March 2010, with the oral presentation Some Generalizations of Quasi-projective Modules.

2. Antalya Algebra Days XIV in Çeşme (International Conference), Çeşme, İzmir, Turkey, 15-20 May 2012, with the oral presentation SGQ-projective Modules. 
3. International Conference on Algebra in Honour of Patrick F. Smith and John Clark's 70th Birthdays, Balıkesir, Turkey, 12-15 August 2013, with the oral presentation Direct Sums of Semi-projective Modules.

4. Categorical Methods in Associative Algebras Seminars, University of Granada, Granada, Spain, 4 October 2013, with the oral presentation Some Generalizations of Quasi-projective Modules.

5. I gave a seminar at 30 January 2014 in Almeria University, Spain with the oral presentation about my thesis.

6. International Conference on Algebra and Number Theory, ICA 2014, Samsun, Turkey, 05-08 August 2014, with the oral presentation A Study on Formal Triangular Matrix Rings. 